\title{
Lazaroa
}

ISSN-e 1988-3307

\section{Phytosociological framework and conservation value of supratemperate riparian birch forest of the Northwestern Iberian Peninsula}

\author{
Manuel Antonio Rodríguez-Guitián ${ }^{1}$, Carlos Real $^{2}$, Rosa Romero Franco ${ }^{1} \&$ Alejandro Álvarez-Hurtado ${ }^{1}$
}

Received: 5 May 2017 / Accepted: 2 October 2017

\begin{abstract}
We studied the downy birch forests that grow in the headwaters of rivers in the mountains in NW Iberian Peninsula and their differences from other previously described birch forests. To do this, an ordination analysis was done on a database of 342 relevés (144 unpublished). As a result, four riparian forest associations were recognized in the territory, all of them belonging to the suballiance Saxifrago spathularis-Fraxinenion excelsioris (Hyperico androsaemiAlnion glutinosae, Alno-Fraxinetalia excelsioris, Salici-Populetea). Two associations (Carici reuterianae-Betuletum celtibericae and Violo palustris-Betuletum pubescentis) have been previously described, but the other two (Chaerophyllo hirsuti-Betuletum pubescentis and Valeriano officinalis-Betuletum pubescentis) are described here for the first time. We characterized their floristic composition, ecological features and distribution, and evaluated their conservation value under the criteria of the 92/43/CEE Community Directive, and with regard to national and regional lists of protected species.
\end{abstract}

Keywords: Betula pubescens; riparian forests; conservation value; Habitat Directive; NW Spain; N Portugal.

[es] Encuadre fitosociológico y valor de conservación de los abedulares riparios supratemplados del noroeste de la Península Ibérica

Resumen. En este trabajo se aborda el estudio de las comunidades de abedulares riparios presentes en los tramos de cabecera de diversos macizos montañosos del extremo NW Ibérico y su diferenciación frente a otros tipos de abedulares previamente descritos. Para ello se ha aplicado un análisis de ordenación a una base de datos conformada por 342 inventarios (144 inéditos). Como resultado, se reconocen cuatro asociaciones vegetales integradas en la subalianza Saxifrago spathularis-Fraxinenion excelsioris (Hyperico androsaemi-Alnion glutinosae, AlnoFraxinetalia excelsioris, Salici-Populetea). Dos de ellas (Carici reuterianae-Betuletum celtibericae y Violo palustris-Betuletum pubescentis) eran previamente conocidas mientras que las otras dos (Chaerophyllo hirsutiBetuletum pubescentis and Valeriano officinalis-Betuletum pubescentis) se dan a conocer aquí por primera vez. Para cada una de ellas se precisa su composición florística, ecología y distribución biogeográfica y se discute su valor de conservación en relación a los criterios establecidos en la Directiva Comunitaria 92/43/CEE así como a los catálogos nacionales y regionales de especies protegidas.

Palabras clave: Betula pubescens; bosques riparios; valor de conservación; Directiva Hábitats; NW España; N Portugal.

\section{Introduction}

Betula pubescens Erhr. (downy birch), the birch growing in the northwestern extreme of the Iberian Peninsula, has a very wide ecological amplitude with regard to climatic constraints (López González, 2001; Ruíz de la Torre, 2006), which allows it to be part of many plant communities. It also tolerates waterlogging and successfully colonizes river banks, particularly in higher terrains where other species common in such environments, like alder (Alnus glutinosa Gaertn.) and common ash (Fraxinus excelsior L.), cannot grow due to low winter temperatures.

\footnotetext{
1 Departament of Crop Production. Escola Politécnica Superior, Universidade de Santiago de Compostela. Campus Universitario, s/n. E-27002 Lugo (Spain). GI-1934 Territorio \& Biodiversidade. E-mail: manuelantonio.rodriguez@usc.es

2 Ecology Unit, Departament of Functional Biology, Escola Politécnica Superior, Universidade de Santiago de Compostela. Campus Universitario, s/n. E-27002 Lugo (Spain).
} 
Gonçalves Aguiar (2000) explicitly recognized the existence of riparian birch forests in the NW Iberian mountains for the first time. He described a community dominated by Salix atrocinerea and Betula celtiberica from the mountains of Montesinho Natural Park (NE Portugal). Afterwards, Honrado et al. (2003) described the association Carici reuterianae-Betuletum celtibericae from the Peneda-Gerês Mountains (NW Portugal), and Rodríguez Guitián (2010) described the association Violo palustris-Betuletum pubescentis from the Northern Galician Mountains and the neighboring ranges of NW Asturias (Figure 1).

Recently, other authors (Silva-Pando, 2009; Rodríguez Guitián et al., 2013, 2014; González de Paz, 2012) interpreted that the riparian birch forests of the $\mathrm{W}$ Cantabrian Range and the Galician-Duriensian ranges belong to the subassociation salicetosum atrocinereae of the climatophilous orocantabrian birch forests (ass. Luzulo henriquesii-Betuletum celtibericae). However, when Izco et al. (1986) described this subassociation, they did not explicitly mentioned any particular relation with streams or water courses.

Other studies suggested that riparian birch forests could be present in other mountainous areas of NW Iberia, such as the Central Massif ofOurense (central-south Galicia), the Sanabria mountais and the Teleno Range (NW Castilla \& León region), but neither their floristic composition nor their syntaxonomic status were explicitly discussed (cf. Castroviejo, 1977; Morla Juaristi, 1985; Navarro Andrés et al., 1986; García López et al., 1992; Romero Rodríguez \& Romero Cuenca, 1996; Gómez Manzaneque, 1997; Lara et al., 2004; Rodríguez Guitián \& Bariego Hernández, 2009).

In a recent review, Biurrun et al. (2016) proposed the inclusion of the riparian birch forests from the mountains among Galicia, Castilla \& León and N Portugal, from the western Cantabrian Range, and from the Moncayo Massif (Iberian Range) in a single association: Carici reuterianae-Betuletum celtibericae. This proposal, which is strongly homogenizing from both biogeographic and phytosociological points of view, is not coherent with the observed multiplicity of riparian forests in the Cantabrian-Atlantic and Orocantabric territories of the Iberian Peninsula (16 associations, Rivas-Martínez et al., 2002; Rivas-Martínez, 2011; Biurrun et al., 2016).
In view of this state of affairs, we decided to collect more data in order to clarify the floristic composition, the phytosociological status, the distribution in the study area, and their connections with other climatophilous, edaphic-higrophilous and serial birch forests. This information will be used to discuss the conservation value of these forests.

\section{Material and methods}

\section{Study area}

The study area comprises the main mountain ranges shared by Galicia, Asturias, Castilla \& León (NW Spain) and N Portugal, as well as the ranges of middle altitude (700-1200 $\mathrm{m}$ asl) constituting the Central and Northern Galician Mountains and NW Asturian Mountains (Figure 1). These territories are mainly siliceous and, following the bioclimatic classification of Rivas-Martínez (2007), most of them belong to the supratemperate bioclimatic horizon (cf. Rodríguez Guitián \& Ramil Rego, 2007). With regard to the biogeographical distribution of the relevés (following the proposal of Rodríguez Guitián \& Ramil-Rego, 2008), they belong to the Galician-Asturian, Inner Galician and Galician-Portuguese sectors of the Cantabro-Atlantic subprovince and to the Western Orocantabrian and Galician-Duriensian sectors of the Orocantabrian subprovince, all of them in the Eurosiberian Region (Figure 1).

\section{Data collection}

New floristic relevés were collected at 144 locations following the Zürich-Montpelllier phytosociological method (Braun-Blanquet, 1979). Information about altitude, slope, exposition and lithology of each plot was also collected. A number of relevés previously published as climatophilous (65), serial (44) or riparian (89) birch forests were incorporated to the data set which finally contained 342 relevés.

\section{Taxonomical considerations}

In order to homogenize the nomenclature we followed the proposals of Flora Iberica (Castroviejo, 1986-2013) and Flora Europaea (Tutin et al., 1964-1980), except for the genera Adenostyles Cass. and Festuca L., for which we followed the criteria of Dillenberger \& 
Kadereit (2012) and Devesa et al. (2013) respectively. We keep the name Betula pubescens Erhr. used in Flora Iberica (http://www. floraiberica.es/miscelania/cambios nomen. php) because: a) in our field work, we repeatedly observed a large variability (both between and within individuals) in the morphological features that several authors proposed as distinctive between Betula pubescens Erhr. and Betula celtiberica Roth. \& Vasc. (Peinado \& Moreno, 1989; Ashburner \& McAllister, 2013); this is in agreement with the observations of other authors (Moreno \& Peinado, 1990; Gómez Manzaneque, 1997; López González, 2001; Ruíz de la Torre, 2006); b) the maps and comments in Ashburner \& McAllister (2013) about the distribution of birches in the NW Iberian Peninsula restrict their presence to the Cantabrian Range but, actually, native birch populations do exist in all mountainous areas of this territory, as many previous studies showed; c) these authors also hypothesized that isolation of the populations of birches in the Cantabrian Range caused their genetic differentiation and that this justifies the existence of only one birch taxa (Betula celtiberica Roth. \& Vasc.) in this area, but this isolation cannot be observed at present. Moreover, paleoenvironmental data about the main tree species of this area during the Pleistocene and Holocene (Ramil-Rego et al., 2011) do not support this hypothesis; and d) recent genetic studies of european birches (Tsuda et al., 2017) including samples from the NW Iberian Peninsula (Devesa da Rogueira in the Courel Range), the Central Range and the Iberian Range, concluded that all these samples belonged to $B$. pubescens.

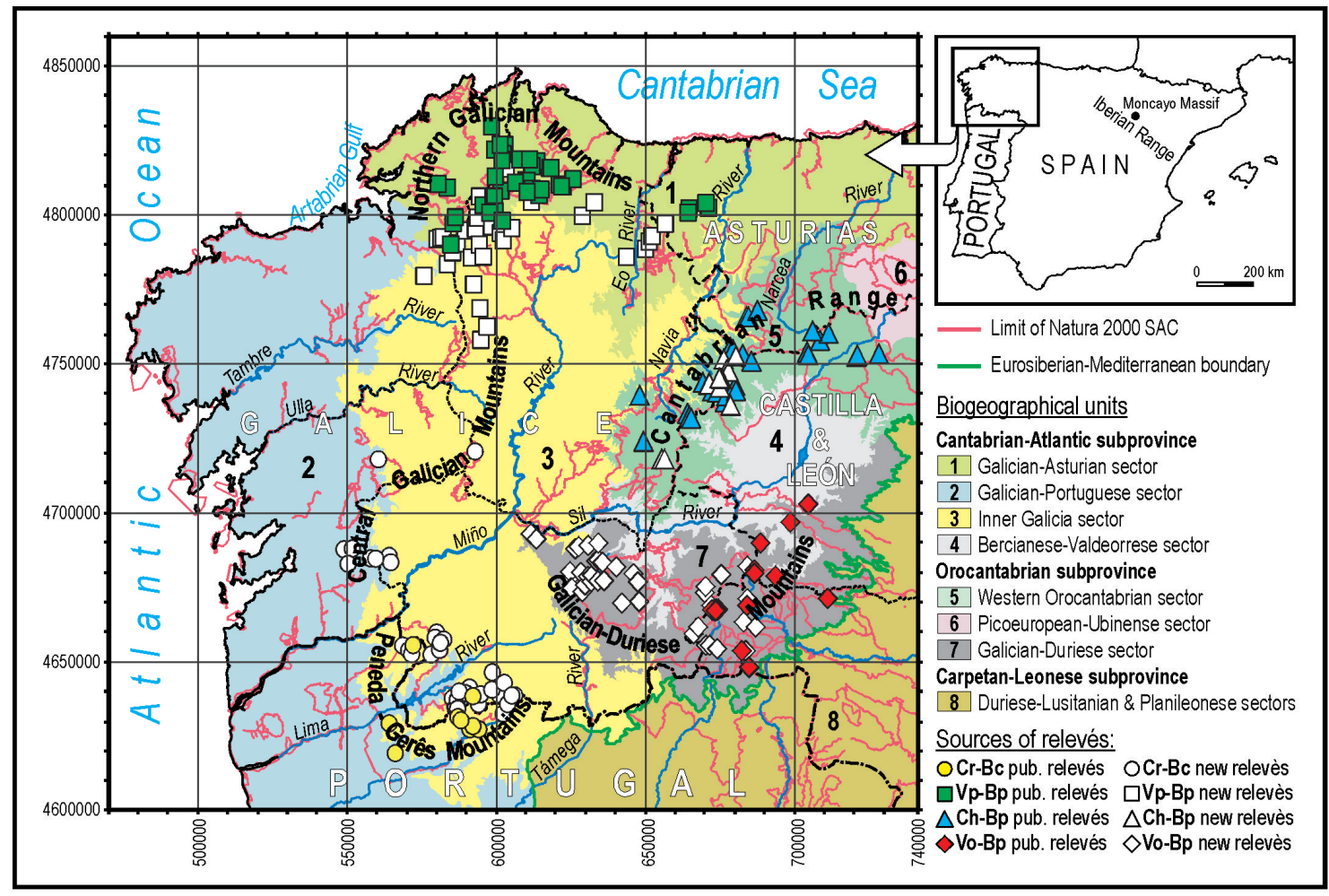

Figure 1. Map of the study area showing the locations of the relevés. Limits of biogeographical units adapted from Rodríguez Guitián \& Ramil Rego (2008) and Rivas-Martínez et al. (2014). Limits of Natura 2000 SAC were taken from Natura 2000 Network Viewer (http://natura2000.eea.europa.eu). Acronyms of associations: Cr-Bc: Carici reuterianae-Betuletum celtibericae; Vp-Bp: Violo palustris-Betuletum pubescentis; Ch-Bp: Chaerophyllo hirsuti-Betuletum pubescentis; Vo-Bp: Valeriano officinalis-Betuletum pubescentis. 
In the case of Viola palustris L., we maintained the nomenclature used in our previous studies (Rodríguez Guitián, 2010; Rodríguez Guitián et al., 2014), without using the infraspecific taxa recognized by several authors (Valentine et al., 1968; Fernández Casado \& Nava Fernández, 2015) because we observed a large variability in the characters differentiating subspecies juresii (Link ex K. Wein) Becker ex Coutinho from the typical subspecies. We followed Guerra \& Cros (2006-2015) for the bryophyte nomenclature. For the syntaxonomical discussion we used the hierarchical scheme and groups of differential species for upper units proposed by Mucina et al. (2016) and Biurrun et al. (2016), respectively.

\section{Multivariate analysis}

In order to establish the floristic relationships between the studied birch forests, the data base was submitted to multivariate analysis. The plants determined at genus level were not included in the analysis. Neither were the bryophytes, which were not recorded in the majority of the previous studies.

The first step of the analysis was to transform the original Braun-Blanquet cover-abundance scale into a numerical scale ranging from 1 to 9 (van der Maarel, 1979). These transformed data were employed to calculate a distance matrix between relevés using the binomial distance (Willis \& Anderson, 2003; Oksanen et al., 2015). A non-metric multidimensional scaling of the distance matrix was done using the procedure metaMDS in the $\mathrm{R}$ package vegan (Oksanen et al., 2015; R Core Team, 2015). The procedure was run 50 times using random initial configurations to arrive to a solution with the least stress.

Prior to the NMDS analysis we studied the influence of the species on the ordination results by deleting each species in turn and calculating a new distance matrix. The correlations (Mantel test) between the original and the pruned matrices allowed us to estimate the influence of each species on the distance structure, sort them from least to most influencing, and finally delete them successively to observe their joint influence on the distance matrix as before. We observed that deleting the species present in four or less relevés (342 in total) did not produce relevant changes in the distance matrix $\left(r^{2}=0,986\right)$. Therefore, 238 out of 448 species were retained, which greatly facilitated the posterior analysis of the relationships between the species and the ordination results. We verified that the elimination of species in the small groups of relevés from the Xurés/ Gerês Mountains and the Moncayo Range (5 and 6 relevés respectively) did not modify their placement in the ordination results.

\section{Conservation value}

Natural habitat types list of the Annex I and plant species listed in annexes II and IV of the Council Directive 92/43/EEC as well as the Spanish and Portuguese national list and Galician, Asturian and Castilian-Leonese regional lists of protected species were used to establish the conservation value of the studied forests.

\section{Results and Discussion}

\section{Ordination results}

Figure 2 shows the position of the relevés in the planes defined by the NMDS axis 1 and 2, and 1 and 3 . The scatter plots at the sides show the relationships between axis 1 and altitude, axis 2 and latitude (UTM-Y coordinates) and axis 3 and the number of taxa in the relevés. Longitude (UTM-X coordinates) was correlated with altitude (and axis 1) because the highest mountains lie in the westernmost part of the study area. The relationships, albeit clear, are not linear. These relationships indicate that there is a biogeographic component in the floristic structure of the data set.

The influence of species richness on the ordination results is interesting. It shows that most bibliographic relevés have relatively low species richness. In fact, these relevés contain between 7 and 59 taxa while the new ones contain between 22 and 73 .

\section{Differences between riparian and other birch forests}

The NMDS scatter plots (Figure 2) show that the climatophilous and seral birch forests (grey symbols) occupy a small area of the ordination space, forming two compact and close groups, while the riparian forests (new and published, white symbols) cover the entire ordination space with a more disperse distribution. This indicates that there are clear floristic differences between the riparian and non-riparian forests, 
and that the former are more floristically diverse. Appendix 1 shows the relative and absolute frequencies of apparition of each species in these two groups of forests. In short, 70 taxa $(30,2 \%)$ were present only in the riparian forests and another $33(14,2 \%)$ were found in more than $90 \%$ of the cases. The most common were (* denotes exclusive taxa): Adenostyles pyrenaica, Ajuga reptans, Angelica major, Aquilegia dichroa, Arrhenatherum bulbosum, Athyrium filix-femina, Brachypodium rupestre, Caltha palustris, Cardamine pratensis*, Carex laevigata, Carex reuteriana*, Centaurea nigra, Chrysosplenium oppositifolium*, Cirsium palustre, Crocus serotinus*, Dactylis glomerata, Deschampsia subtriflora, Epilobium obscurum*, Euphorbia dulcis, Galium broterianum*, Galium palustre*, Heracleum sphondylium, Juncus effusus*, Lastrea limbosperma, Lathyrus linifolius*, Lonicera hispanica*, Narcissus asturiensis*, Oenanthe crocata*, Osmunda regalis*, Peucedanum lancifolium*, Primula acaulis, Prunella vulgaris*, Ranunculus ficaria*, Ranunculus repens*, Rumex acetosa, Salix atrocinerea, Saxifraga lepismigena*, Scrophularia auriculata*, Valeriana officinalis*, Viola palustris and Wahlenbergia hederacea*.
On the other hand, only Agrostis castellana and Myosotis sylvatica were exclusively associated to non-riparian forests and Lonicera periclymenum showed a strong preference for them.

The riparian forests of the Moncayo Massif (black circles) are close to (and in between) the climatophilous and serial forests in the ordination results. Therefore, they are not similar to the new relevés collected for this study. This might be caused by the small number of taxa in these relevés (between 17 and 24), the lack of many hygrophilous taxa usually associated with the riparian forests of the NW of the Iberian Peninsula, and the presence of many species common in the non-riparian forests.

Neither the hygrophilous variant of the association Holco mollis-Betuletum celtibericae (light grey squares) nor the subass. salicetosum atrocinereae of the association Luzulo henriquesii-Betuletum celtibericae (dark grey diamonds) were very similar to the studied forests (see Table 1). Only the latter group should show some affinity with the riparian birch forests of the western Cantabrian Mountains investigated in this paper, but they lack many of the aforementioned riparian species made them floristically closer to the non-riparian forests.

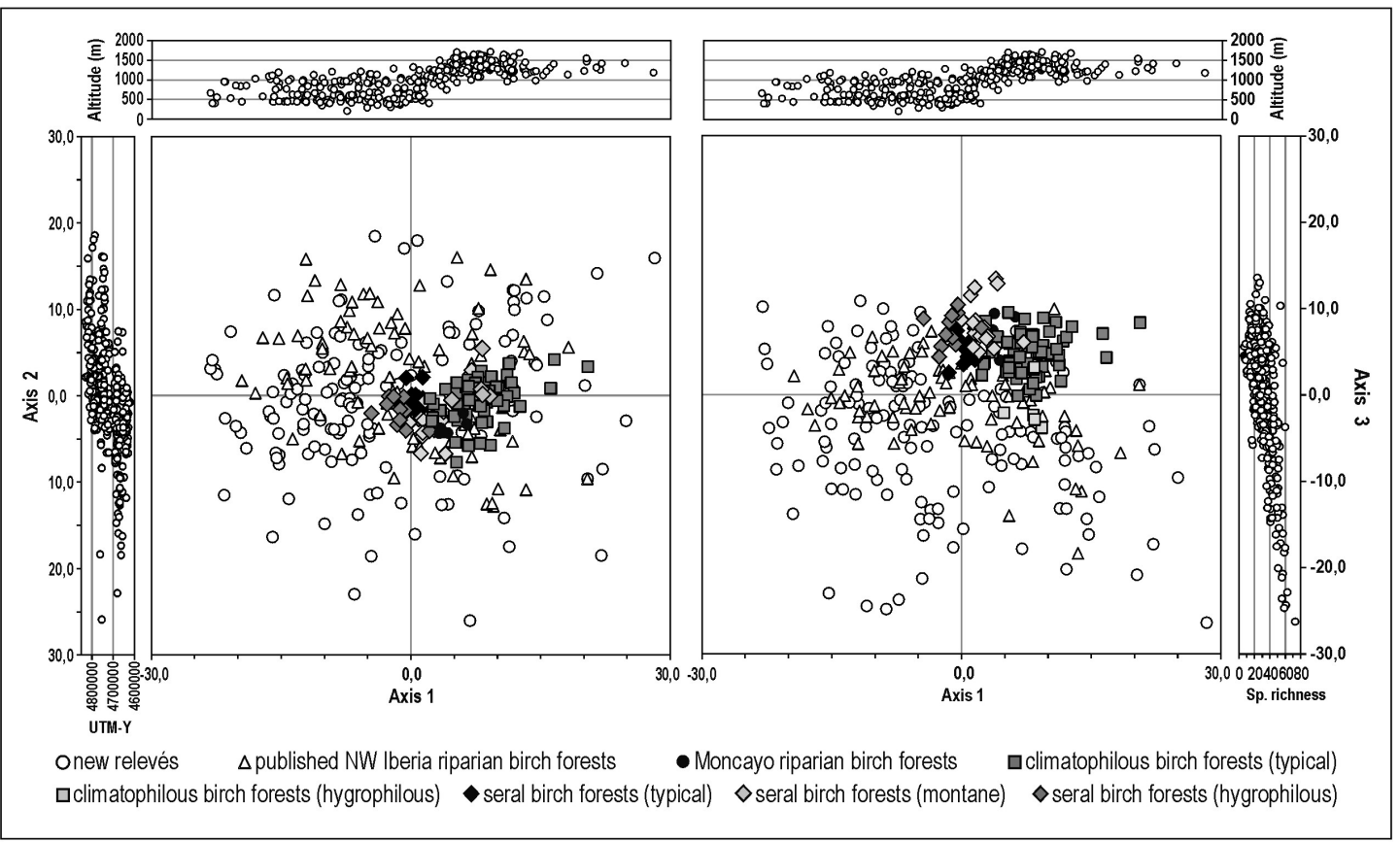

Figure 2. Ordination diagrams for the first three axis of the NMDS results. The side graphs show the relationship between the axes and the variables altitude (axis 1), latitude (as UTM-Y coordinate, axis 2) and species richness (axis 3). 
The floristic variability of the riparian birch forests

The relationship among the geographic variables and the ordination axis and the existence of two previously described associations of riparian birch forests, suggested grouping the relevés by their biogeographic origin. Figure 1 shows the distribution of the relevés in four groups: N Galicia and NW Asturias forests (squares, labeled as Vp-Bp), W Cantabrian Range forests (triangles, Ch-Bp), Galician-Duriensian forests (diamonds, Vo-Bp) and Central Galician Range and N Portugal forests (circles, $\mathrm{Cr}-\mathrm{Bc}$ ). These groups were also represented in Figure 3, wich contains the NMDS results for axis 1 and 2. The first three groups can be easily recognized in the graph, where there are no or limited overlapping between them. The fourth group $(\mathrm{Cr}-\mathrm{Bc})$ is completely intermixed with the $\mathrm{Vp}-\mathrm{Bp}$ and Vo-Bp groups. We consider that this distribution appears because the relevés in the left side of the graph come from the westernmost ranges ( $\mathrm{N}$ and Central Galician and $\mathrm{N}$ Portugal ranges), of generally lower altitude, which make the relevés to share an elevated number species. These overweighted the influence of the biogeographically restricted species on the ordination results, especially in the case of the $\mathrm{Cr}-\mathrm{Bc}$ group. In this regard, this group plays the role of floristic bridge between the north and south of the study area. Note that the relevés of the Vo-Bp group from higher altitudes (at the right side) do not mix with the $\mathrm{Vp}-\mathrm{Bp}$ or $\mathrm{Cr}-\mathrm{Bc}$ groups.

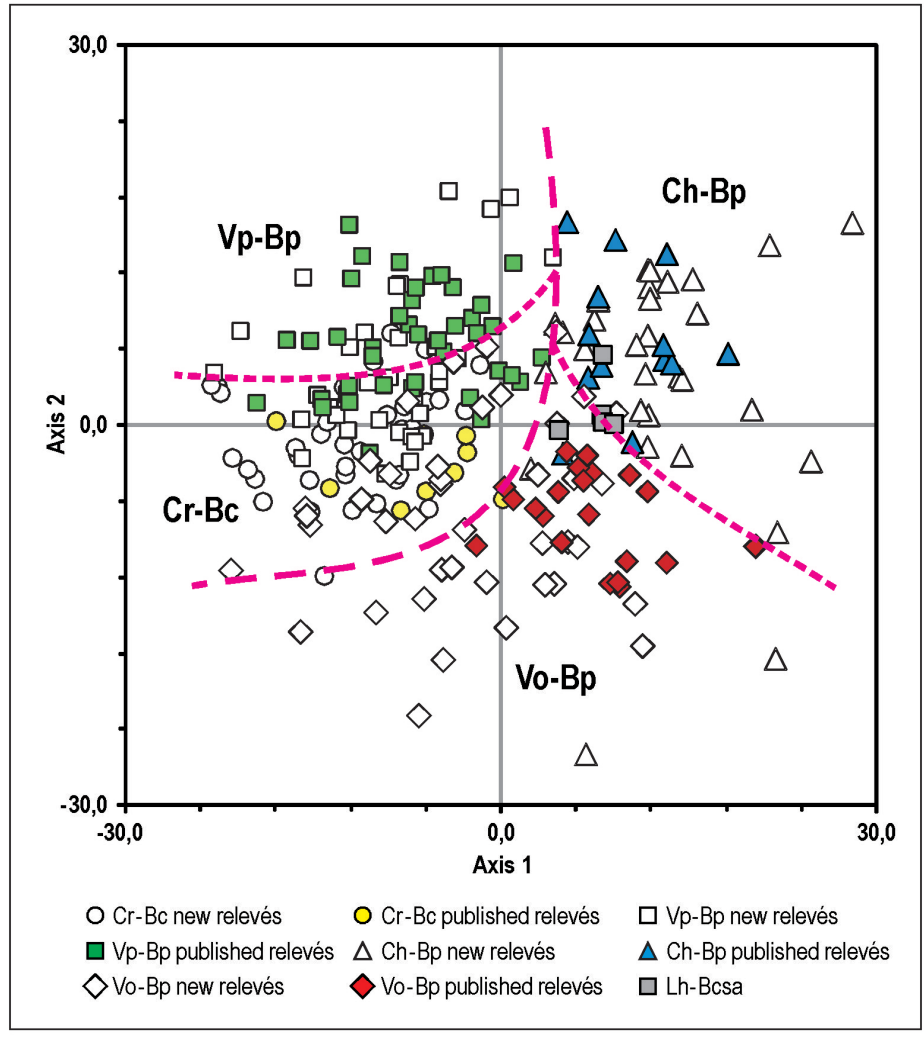

Figure 3. Representation of the four groups of riparian birch forests on the results of the ordination (axes 1 and 2). The relevés of the subass. salicetosum atrocinereae of the ass. Luzulo henriquesii-Betuletum celtibericae (labeled Lh-Bcsa) were also included. 
Table 1. Synthetic table showing the floristic differences between the Norhtwestern riparian birch forests and the other birch forests types considered in this study. Taxa with frequencies greater than $50 \%$ are highlighted in grey. Except for differential taxa, only those present in more than three or four groups were selected.

\begin{tabular}{|c|c|c|c|c|c|c|c|c|c|c|c|c|c|c|c|c|}
\hline \multirow[t]{3}{*}{ Altitude (m asl) } & Maximum & 1205 & 1050 & 770 & 980 & 1460 & 1405 & 1370 & 1625 & 1640 & 1250 & 1270 & 1710 & 790 & 1140 & 490 \\
\hline & Average & 897 & 852 & 530 & 532 & 1205 & 1235 & 1284 & 1068 & 1372 & 1200 & 1198 & 1446 & 564 & 996 & 421 \\
\hline & Minimum & 400 & 470 & 400 & 205 & 950 & 1020 & 1200 & 650 & 1190 & 1090 & 1110 & 1200 & 400 & 850 & 360 \\
\hline \multirow[t]{3}{*}{ Number of taxa } & Maximum & 66 & 47 & 58 & 49 & 75 & 61 & 25 & 68 & 44 & 24 & 29 & 36 & 17 & 29 & 23 \\
\hline & Average & 43.7 & 31.1 & 37.3 & 35.3 & 47.0 & 40.2 & 24.4 & 43.8 & 29.6 & 20.5 & 22.6 & 20.8 & 12.5 & 19.6 & 17.4 \\
\hline & Minimum & $\begin{array}{l}27 \\
\mathrm{Cr}-\end{array}$ & $\begin{array}{l}20 \\
\mathrm{Cr}-\end{array}$ & $\begin{array}{c}24 \\
\mathrm{Vp}-\end{array}$ & $\begin{array}{l}16 \\
\text { Vp- }\end{array}$ & $\begin{array}{l}25 \\
\mathrm{Ch}-\end{array}$ & $\begin{array}{c}18 \\
\text { Ch- }\end{array}$ & $\begin{array}{l}23 \\
\text { Lh- }\end{array}$ & $\begin{array}{l}23 \\
\text { Vo- }\end{array}$ & $\begin{array}{l}18 \\
\text { Vo- }\end{array}$ & $\begin{array}{c}17 \\
\text { Rc- }\end{array}$ & $\begin{array}{l}12 \\
\text { Ej- }\end{array}$ & $\begin{array}{c}9 \\
\text { Lh- }\end{array}$ & $\begin{array}{c}8 \\
\mathrm{Hm}-\end{array}$ & $\begin{array}{c}13 \\
\mathrm{Hm}-\end{array}$ & $\begin{array}{c}13 \\
\mathrm{Hm}-\end{array}$ \\
\hline \multicolumn{2}{|c|}{ Group } & $\begin{array}{l}\mathrm{Bc} \\
1 \mathrm{a}\end{array}$ & $\begin{array}{l}\mathrm{Bc} \\
1 \mathrm{~b}\end{array}$ & $\begin{array}{l}\text { Bp } \\
2 \mathrm{a}\end{array}$ & $\begin{array}{l}\mathrm{Bp} \\
2 \mathrm{~b}\end{array}$ & $\begin{array}{l}\text { Bp } \\
3 \mathrm{a}\end{array}$ & $\begin{array}{l}\mathrm{Bp} \\
3 \mathrm{~b}\end{array}$ & $\begin{array}{c}\text { Bcsa } \\
4\end{array}$ & $\begin{array}{l}\mathrm{Bp} \\
5 \mathrm{a}\end{array}$ & $\begin{array}{l}\mathrm{Bp} \\
5 \mathrm{~b}\end{array}$ & $\underset{6}{\operatorname{Savm}}$ & $\begin{array}{c}\mathrm{Bc} \\
7\end{array}$ & $\begin{array}{c}\text { Bct } \\
8\end{array}$ & $\begin{array}{l}\text { Bct } \\
9 \mathrm{a}\end{array}$ & $\begin{array}{l}\mathrm{Bcm} \\
9 \mathrm{~b}\end{array}$ & $\begin{array}{c}\text { Bch } \\
9 \mathrm{c}\end{array}$ \\
\hline \multicolumn{2}{|c|}{ Number of relevés } & 37 & 9 & 35 & 40 & 30 & 13 & 5 & 42 & 21 & 6 & 5 & 55 & 16 & 17 & 11 \\
\hline \multicolumn{2}{|c|}{ N. relevé } & 1 & 2 & 3 & 4 & 5 & 6 & 7 & 8 & 9 & 10 & 11 & 12 & 13 & 14 & 15 \\
\hline \multicolumn{17}{|c|}{$\mathrm{E}_{1}(>4,0 \mathrm{~m})+\mathrm{E}_{2}(1,5-4,0 \mathrm{~m})$} \\
\hline \multicolumn{2}{|c|}{ Betula pubescens } & 94.6 & 100 & 97.1 & 97.5 & 100 & 100 & 100 & 97.6 & 100 & 100 & 100 & 100 & 100 & 100 & 100 \\
\hline \multicolumn{2}{|l|}{ Erica arborea } & 89.2 & 88.9 & 80.0 & 80.0 & 73.3 & 76.9 & 100 & 78.6 & 95.2 & 66.7 & 100 & 83.6 & 43.8 & 64.7 & - \\
\hline \multicolumn{2}{|l|}{ Salix atrocinerea } & 97.3 & 100 & 94.3 & 97.5 & 80.0 & 84.6 & 80.0 & 92.9 & 61.9 & 100 & 60.0 & 3.6 & 12.5 & 5.9 & 45.5 \\
\hline \multicolumn{2}{|l|}{ Sorbus aucuparia } & 5.4 & - & 8.6 & 10.0 & 86.7 & 84.6 & 100 & 64.3 & 95.2 & 33.3 & 100 & 98.2 & - & 82.4 & - \\
\hline \multicolumn{2}{|l|}{ Frangula alnus } & 56.8 & 33.3 & 77.1 & 50.0 & 26.7 & 15.4 & - & 64.3 & 33.3 & 100 & 40.0 & 1.8 & 12.5 & 5.9 & 72.7 \\
\hline \multicolumn{2}{|l|}{ Ilex aquifolium } & 10.8 & - & 31.4 & 25.0 & 73.3 & 76.9 & 40.0 & 28.6 & 42.9 & 66.7 & 60.0 & 50.9 & 18.8 & 47.1 & 9.1 \\
\hline Corylus avellana & & 13.5 & 22.2 & 20.0 & 32.5 & 80.0 & 84.6 & 60.0 & 54.8 & 19.0 & 50.0 & - & 30.9 & - & 29.4 & 9.1 \\
\hline Genista florida & & 37.8 & 33.3 & 5.7 & 2.5 & 26.7 & 30.8 & 20.0 & 40.5 & 38.1 & - & 60.0 & 32.7 & - & 41.2 & - \\
\hline Quercus pyrenaic & & 45.9 & 22.2 & 5.7 & - & 6.7 & - & - & 57.1 & 28.6 & - & - & 1.8 & - & 17.6 & 18.2 \\
\hline Acer pseudoplato & inus & 2.7 & 11.1 & 14.3 & 7.5 & 43.3 & 30.8 & - & 26.2 & 4.8 & - & - & 16.4 & - & 47.1 & - \\
\hline Cytisus scoparius & & 13.5 & 22.2 & 22.9 & 17.5 & 3.3 & 7.7 & - & 11.9 & 14.3 & - & - & 3.6 & 12.5 & 17.6 & 18.2 \\
\hline Castanea sativa & & 10.8 & 11.1 & 14.3 & 20.0 & 3.3 & - & - & 7.1 & - & - & - & - & 62.5 & 29.4 & 36.4 \\
\hline Rosa gr. canina & & 32.4 & - & 2.9 & 2.5 & 6.7 & 23.1 & - & 35.7 & - & - & - & 3.6 & - & - & - \\
\hline Crataegus monog & syna & 27.0 & 22.2 & - & 7.5 & 3.3 & - & - & 14.3 & 9.5 & - & - & 3.6 & - & 5.9 & 36.4 \\
\hline Fraxinus excelsic & & - & - & 11.4 & 12.5 & 26.7 & - & - & 4.8 & - & 16.7 & - & 1.8 & 6.3 & 11.8 & 9.1 \\
\hline Alnus glutinosa & & 5.4 & - & 25.7 & 7.5 & 3.3 & - & - & 2.4 & 9.5 & - & - & - & - & - & 54.5 \\
\hline Sambucus nigra & & 8.1 & - & 20.0 & 12.5 & - & - & - & 4.8 & - & - & - & - & 6.3 & 11.8 & 9.1 \\
\hline Taxus baccata & & - & - & - & - & 6.7 & - & 20.0 & 2.4 & - & - & 40.0 & 20.0 & - & - & - \\
\hline Prunus avium & & - & 22.2 & 2.9 & - & 10.0 & - & - & 7.1 & - & - & - & - & - & 11.8 & - \\
\hline $\mathrm{E}_{3}(<1,5 \mathrm{~m})$ & & & & & & & & & & & & & & & & \\
\hline $\begin{array}{l}\text { Differential grou } \\
\mathrm{Cr}-\mathrm{Bc}\end{array}$ & p species & & & & & & & & & & & & & & & \\
\hline Anemone albida & & 37.8 & 33.3 & - & - & - & - & - & - & - & - & 80.0 & - & - & - & - \\
\hline Laserpitium thalt & ctrifolium & 5.4 & 11.1 & - & - & - & - & - & - & - & - & 40.0 & - & - & - & - \\
\hline Echium lusitanic & & 2.7 & 11.1 & - & - & - & - & - & - & - & - & - & - & - & - & - \\
\hline Hyacinthoides po & ivae & 32.4 & - & - & - & - & - & - & - & - & - & 20.0 & - & - & - & - \\
\hline $\begin{array}{l}\text { Agrostis } x \text { fouilla } \\
\text { Vp-Bp }\end{array}$ & & - & 44.4 & - & - & - & - & - & - & - & - & - & - & - & - & - \\
\hline Senecio nemoren & & - & - & 60.0 & 60.0 & - & - & - & - & - & - & - & - & - & - & 63.6 \\
\hline Anthoxanthum a & narum & - & - & 20.0 & 12.5 & - & - & - & - & - & - & - & 7.3 & 6.3 & 11.8 & - \\
\hline Dryopteris aemu & & - & - & 17.1 & 32.5 & - & - & - & - & - & - & - & - & - & - & - \\
\hline Stachys officinali & & - & - & 8.6 & 20.0 & - & - & - & - & - & 33.3 & - & - & - & - & - \\
\hline Valeriana dioica & & - & - & 11.4 & 2.5 & - & - & - & - & - & - & - & - & - & - & 45.5 \\
\hline Myosotis martini & & - & - & 17.1 & 5.0 & - & - & - & - & - & - & - & - & - & - & - \\
\hline Lythrum salicaric & & - & - & 14.3 & 5.0 & - & - & - & - & - & - & - & - & - & - & - \\
\hline Senecio aquaticu & & - & - & 11.4 & 5.0 & - & - & - & - & - & - & - & - & - & - & - \\
\hline Erica mackaiana & & - & - & 5.7 & 2.5 & - & - & - & - & - & - & - & - & 6.3 & - & - \\
\hline $\begin{array}{l}\text { Cytisus commuta } \\
\text { Ch-Bc }\end{array}$ & & - & - & 5.7 & - & - & - & - & - & - & - & - & - & - & - & - \\
\hline Fagus sylvatica & & - & - & - & - & 13.3 & 15.4 & 20.0 & - & - & 83.3 & 20.0 & 9.1 & - & 11.8 & - \\
\hline Quercus petraea & & - & - & - & - & 26.7 & 46.2 & - & - & - & - & - & 7.3 & - & 5.9 & - \\
\hline Daphne laureola & & - & - & - & - & 13.3 & 7.7 & 80.0 & - & - & - & - & 5.5 & - & 11.8 & - \\
\hline Sanicula europae & & - & - & - & - & 20.0 & 15.4 & - & - & - & - & - & 5.5 & - & 5.9 & - \\
\hline Milium effusum & & - & - & - & - & 16.7 & 15.4 & - & - & - & - & - & 3.6 & - & - & - \\
\hline Cicerbita plumie & & - & - & - & - & 23.3 & 15.4 & - & - & - & - & - & 3.6 & - & - & - \\
\hline Galium rotundifo & lium & - & - & - & - & 6.7 & 7.7 & - & - & - & - & - & 5.5 & - & - & - \\
\hline Stellaria montan & & - & - & - & - & 6.7 & 7.7 & - & - & - & - & - & 3.6 & - & - & - \\
\hline Festuca altissima & & - & - & - & - & 6.7 & 7.7 & 20.0 & - & - & - & - & - & - & - & - \\
\hline Quercus robur $\mathrm{x}$ & Q. petraea & - & - & - & - & 3.3 & 7.7 & - & - & - & - & - & 12.7 & - & 5.9 & - \\
\hline
\end{tabular}




\begin{tabular}{|c|c|c|c|c|c|c|c|c|c|c|c|c|c|c|c|}
\hline Galium odoratum & - & - & - & - & 16.7 & - & - & - & - & - & - & 5.5 & - & - & - \\
\hline Primula columnae & - & - & - & - & 13.3 & - & - & - & - & - & - & 1.8 & - & - & - \\
\hline Veronica montana & - & - & - & - & 10.0 & - & - & - & - & - & - & 3.6 & - & - & 9.1 \\
\hline Helleborus occidentalis & - & - & - & - & 6.7 & - & - & - & - & - & - & 7.3 & - & - & - \\
\hline Astrantia major & - & - & - & - & 30.0 & - & - & - & - & - & - & 3.6 & - & - & - \\
\hline Lasepitium merinoi & - & - & - & - & 16.7 & - & - & - & - & - & - & - & - & - & - \\
\hline Mentha longifolia & - & - & - & - & 10.0 & - & - & - & - & - & - & - & - & - & - \\
\hline Geranium sylvaticum & - & - & - & - & 10.0 & - & - & - & - & - & - & - & - & - & - \\
\hline \multicolumn{16}{|l|}{ Vo-Bp } \\
\hline Viburnum opulus & - & - & - & - & - & - & - & 11.9 & 4.8 & - & - & - & - & - & 9.1 \\
\hline Rosa corymbifera & - & - & - & - & - & - & - & 4.8 & 9.5 & - & - & - & - & - & 9.1 \\
\hline Streptopus amplexifolius & - & - & - & - & - & - & - & 2.4 & 14.3 & - & - & - & - & - & - \\
\hline Stachys sylvatica & - & - & - & - & - & - & - & 2.4 & 14.3 & - & - & - & - & - & - \\
\hline Pimpinella major & - & - & - & - & - & - & - & 9.5 & - & - & - & - & - & - & - \\
\hline \multicolumn{16}{|l|}{ Rc-Sa } \\
\hline Rubus plicatus & - & - & - & - & - & - & - & - & - & 100 & - & - & - & - & - \\
\hline Lysimachia vulgaris & - & - & 2.9 & 2.5 & - & - & - & - & - & 83.3 & - & - & - & - & - \\
\hline Carex divulsa & - & - & - & 2.5 & - & - & - & - & - & 83.3 & - & - & - & - & - \\
\hline Erica vagans & - & - & - & 2.5 & - & - & - & - & - & 50.0 & - & - & - & - & 27.3 \\
\hline Sorbus torminalis & - & - & - & - & - & - & - & - & - & 33.3 & - & - & - & - & - \\
\hline Ranunculus acris & - & - & - & - & - & - & - & - & - & 16.7 & - & - & - & - & - \\
\hline Sambucus racemosa & - & - & - & - & - & - & - & - & - & 16.7 & - & - & - & - & - \\
\hline \multicolumn{16}{|l|}{$\mathrm{Vp}-\mathrm{Bp}+\mathrm{Cr}-\mathrm{Bc}$} \\
\hline Osmunda regalis & 16.2 & 44.4 & 31.4 & 35.0 & - & - & - & - & - & - & - & - & - & - & - \\
\hline Cytisus striatus & 16.2 & 11.1 & 2.9 & - & - & - & - & - & - & - & - & - & 6.3 & - & - \\
\hline Crocus serotinus & 54.1 & - & 28.6 & 47.5 & - & - & - & - & - & - & - & - & - & - & - \\
\hline Laurus nobilis & 5.4 & - & 2.9 & 2.5 & - & - & - & - & - & - & - & - & - & - & - \\
\hline Senecio doria & 2.7 & 11.1 & 2.9 & - & - & - & - & - & - & - & - & - & - & - & - \\
\hline \multicolumn{16}{|l|}{$\mathrm{Vp}-\mathrm{Bp}+\mathrm{Ch}-\mathrm{Bc}$} \\
\hline Valeriana montana & - & - & 2.9 & - & 56.7 & 46.2 & 60.0 & - & - & - & - & 25.5 & - & - & - \\
\hline Valeriana pyrenaica & - & - & 2.9 & 5.0 & 36.7 & 23.1 & 20.0 & - & - & - & - & 1.8 & - & - & - \\
\hline Scrophularia alpestris & - & - & 2.9 & - & 43.3 & 15.4 & - & - & - & 16.7 & - & - & - & - & - \\
\hline Festuca gigantea & - & - & - & 2.5 & 10.0 & - & - & - & - & - & - & - & - & - & - \\
\hline \multicolumn{16}{|l|}{$\mathrm{Cr}-\mathrm{Bc}+\mathrm{Ch}-\mathrm{Bp}$} \\
\hline Ornithogalum pyrenaicum & 5.4 & 11.1 & - & - & 10.0 & - & - & - & - & - & - & - & - & 5.9 & - \\
\hline Polygonum bistorta & 5.4 & - & - & - & 10.0 & - & - & - & - & - & - & - & - & - & - \\
\hline \multicolumn{16}{|l|}{$\mathrm{Cr}-\mathrm{Bc}+\mathrm{Vo}-\mathrm{Bp}$} \\
\hline Aquilegia dichroa & 51.4 & 44.4 & - & - & - & - & - & 42.9 & 47.6 & - & 20.0 & - & - & - & - \\
\hline Galium broterianum & 48.6 & 22.2 & - & - & - & - & - & 33.3 & 19.0 & - & - & - & - & - & - \\
\hline Paradisea lusitanica & 27.0 & 33.3 & - & - & - & - & - & 11.9 & 4.8 & - & - & - & - & - & - \\
\hline Valeriana officinalis & 13.5 & - & - & - & - & - & - & 45.2 & 47.6 & - & - & - & - & - & - \\
\hline Allium scorzonerifolium & 5.4 & 33.3 & - & - & - & - & - & 14.3 & - & - & - & - & - & - & - \\
\hline Thalictrum speciosissimum & 10.8 & 11.1 & - & - & - & - & - & 14.3 & - & - & - & - & - & - & - \\
\hline Calamagrostis arundinacea & 10.8 & 44.4 & - & - & - & - & - & 4.8 & - & - & - & - & - & - & - \\
\hline Myosotis cespitosa & 13.5 & 11.1 & - & - & - & - & - & 7.1 & - & - & - & - & - & - & - \\
\hline Fraxinus angustifolia & 2.7 & 11.1 & - & - & - & - & - & 7.1 & - & - & - & - & - & - & 9.1 \\
\hline Knautia nevadensis & - & 11.1 & - & - & - & - & - & 4.8 & - & - & - & - & - & - & - \\
\hline \multicolumn{16}{|l|}{$\mathrm{Ch}-\mathrm{Bc}+\mathrm{Vo}-\mathrm{Bp}$} \\
\hline Poa nemoralis & - & - & - & - & 50.0 & 38.5 & 60.0 & 54.8 & 71.4 & 83.3 & - & 30.9 & - & 5.9 & - \\
\hline Doronicum pubescens & - & - & - & - & 10.0 & 23.1 & - & 4.8 & 14.3 & - & - & 23.6 & - & - & - \\
\hline Aconitum neapolitanum & - & - & - & - & 26.7 & 15.4 & - & 7.1 & 28.6 & - & - & 1.8 & - & - & - \\
\hline Narcissus pseudonarc. s.1. & - & - & - & - & 6.7 & 15.4 & - & 14.3 & 9.5 & - & - & 1.8 & - & - & - \\
\hline Conopodium majus & - & - & - & - & 3.3 & 23.1 & - & 4.8 & - & - & - & 7.3 & - & 5.9 & - \\
\hline Hieracium sabaudum & - & - & - & - & 13.3 & 7.7 & - & 2.4 & - & - & - & 1.8 & - & 5.9 & - \\
\hline Melica uniflora & - & - & - & - & 16.7 & - & - & 7.1 & 9.5 & 16.7 & - & 7.3 & - & - & - \\
\hline Poa chaixii & - & - & - & - & 16.7 & 30.8 & - & 4.8 & - & - & - & 21.8 & - & - & - \\
\hline Cardamine gallaecica & - & - & - & - & 26.7 & 15.4 & - & 9.5 & 4.8 & - & - & - & - & - & - \\
\hline Prunus padus & - & - & - & - & 6.7 & - & - & 7.1 & 28.6 & - & - & 5.5 & - & - & - \\
\hline Rubus idaeus & - & - & - & - & 13.3 & - & - & 7.1 & 23.8 & - & - & 1.8 & - & - & - \\
\hline Veratrum album & - & - & - & - & 3.3 & - & - & 7.1 & 9.5 & - & - & 9.1 & - & - & - \\
\hline Erythronium dens-canis & - & - & - & - & 3.3 & - & - & 4.8 & 14.3 & - & - & 5.5 & - & - & - \\
\hline Rosa villosa & - & - & - & - & 16.7 & - & - & 26.2 & 9.5 & - & - & - & - & - & - \\
\hline Pyrola minor & - & - & - & - & 13.3 & - & - & 7.1 & 23.8 & - & - & - & - & - & - \\
\hline Polystichum aculeatum & - & - & - & - & 20.0 & - & - & - & 4.8 & - & - & 5.5 & - & - & - \\
\hline
\end{tabular}




\begin{tabular}{|c|c|c|c|c|c|c|c|c|c|c|c|c|c|c|c|}
\hline Helleborus foetidus & - & - & - & - & 13.3 & - & - & - & 14.3 & - & - & - & - & 11.8 & - \\
\hline Epilobium montanum & - & - & - & - & 16.7 & - & - & 2.4 & 9.5 & - & - & - & - & - & - \\
\hline Paris quadrifolia & - & - & - & - & 3.3 & - & - & 7.1 & 9.5 & - & - & - & - & - & - \\
\hline Cystopteris fragilis & - & - & - & - & 3.3 & - & - & 4.8 & 4.8 & - & - & - & - & - & - \\
\hline Epilobium angustifolium & - & - & - & - & 3.3 & - & - & 7.1 & - & - & - & - & - & - & - \\
\hline Ribes alpinum & - & - & - & - & 6.7 & - & - & 2.4 & - & - & - & - & - & - & - \\
\hline Thalictrum minus & - & - & - & - & 6.7 & - & - & - & 23.8 & - & - & - & - & - & - \\
\hline \multicolumn{16}{|l|}{$\mathrm{Vp}-\mathrm{Bp}+\mathrm{Cr}-\mathrm{Bc}+\mathrm{Vo}-\mathrm{Bp}$} \\
\hline Quercus robur & 67.6 & 22.2 & 88.6 & 87.5 & - & - & 20.0 & 28.6 & - & - & 20.0 & 5.5 & 100 & 41.2 & 100 \\
\hline Cirsium filipendulum & 24.3 & 11.1 & 8.6 & 2.5 & - & - & - & 7.1 & - & - & 20.0 & - & 6.3 & - & 18.2 \\
\hline Pyrus cordata & 37.8 & 11.1 & 37.1 & 37.5 & - & - & - & 7.1 & - & - & 20.0 & - & 6.3 & 5.9 & - \\
\hline Galium palustre & 18.9 & - & 28.6 & 7.5 & - & - & - & 7.1 & 9.5 & 16.7 & - & - & - & - & - \\
\hline Molinia caerulea & 13.5 & - & 17.1 & 20.0 & - & - & - & 9.5 & - & 50.0 & 20.0 & - & - & - & - \\
\hline Oenanthe crocata & 51.4 & 55.6 & 54.3 & 55.0 & - & - & - & 26.2 & - & - & - & - & - & - & - \\
\hline Scrophularia auriculata & 13.5 & 22.2 & 31.4 & 12.5 & - & - & - & 4.8 & - & - & - & - & - & - & - \\
\hline Scutellaria minor & 32.4 & - & 5.7 & 5.0 & - & - & - & 19.0 & - & - & - & - & - & - & 18.2 \\
\hline Scrophularia herminii & 8.1 & 11.1 & 5.7 & - & - & - & - & 7.1 & 9.5 & - & - & - & - & - & - \\
\hline Pulmonaria longifolia & 8.1 & - & 11.4 & 12.5 & - & - & - & 2.4 & - & - & - & - & - & - & - \\
\hline \multicolumn{16}{|l|}{$\mathrm{Vp}-\mathrm{Bp}+\mathrm{Ch}-\mathrm{Bc}+\mathrm{Vo}-\mathrm{Bp}$} \\
\hline Melampyrum pratense & 2.7 & - & 11.4 & - & 13.3 & 15.4 & - & 23.8 & 23.8 & 66.7 & - & 52.7 & 6.3 & 52.9 & 9.1 \\
\hline Polygonatum verticillatum & - & - & 5.7 & - & 46.7 & 53.8 & 60.0 & 16.7 & 28.6 & - & - & 18.2 & - & - & - \\
\hline Chaerophyllum hirsutum & - & - & 8.6 & 2.5 & 80.0 & 69.2 & 100 & 4.8 & 4.8 & - & - & 3.6 & - & - & - \\
\hline Adenostyles pyrenaica & - & - & 2.9 & 25.0 & 70.0 & 76.9 & 80.0 & 9.5 & 28.6 & - & - & - & - & - & - \\
\hline Salix caprea & - & - & 2.9 & - & 43.3 & 23.1 & 20.0 & 11.9 & 14.3 & - & - & 5.5 & - & 5.9 & - \\
\hline Aquilegia vulgaris & - & - & - & 5.0 & 43.3 & 7.7 & - & 16.7 & 4.8 & - & - & 9.1 & - & - & - \\
\hline Silene dioica & - & - & 8.6 & 15.0 & 50.0 & 30.8 & - & 33.3 & 14.3 & - & - & 3.6 & - & - & - \\
\hline Ceratocapnos claviculata & - & - & - & 2.5 & 10.0 & 7.7 & - & 7.1 & 19.0 & - & - & 10.9 & - & - & - \\
\hline Carex remota & - & - & 5.7 & 5.0 & 10.0 & 7.7 & - & 2.4 & - & - & - & - & - & - & 18.2 \\
\hline Polystichums & - & - & 2.9 & - & 20.0 & 15.4 & - & 7 & 4.8 & - & - & - & - & 11.8 & - \\
\hline Cardamine pratensis & - & - & 42.9 & 20.0 & 3.3 & 7.7 & - & 4.8 & - & - & - & - & - & - & - \\
\hline Narcissus asturiensis & - & - & 8.6 & 30.0 & 23.3 & 30.8 & - & 2. & - & - & - & - & - & - & - \\
\hline Lysimachia nemorum & - & - & 5.7 & 5.0 & 26.7 & - & - & 2. & - & - & - & - & - & - & - \\
\hline Hypericum androsaemum & - & - & 5.7 & 15.0 & 3.3 & - & - & 2. & - & - & - & - & - & - & - \\
\hline \multicolumn{16}{|l|}{$\mathrm{Cr}-\mathrm{Bc}+\mathrm{Ch}-\mathrm{Bc}+\mathrm{Vo}-\mathrm{Bp}$} \\
\hline Lilium martagon & 2.7 & 11.1 & - & - & 13.3 & 7.7 & - & 9.5 & 28.6 & - & 60.0 & 20.0 & - & - & - \\
\hline Euphorbia hyberna & 13.5 & 11.1 & - & - & 56.7 & 53.8 & 20.0 & 19.0 & 19.0 & - & - & 23.6 & - & - & - \\
\hline Ranunculus platanifolius & 8.1 & - & - & - & 50.0 & 53.8 & - & 31.0 & 38.1 & - & 20.0 & 3.6 & - & - & - \\
\hline Festuca $m$ & 2.7 & - & - & - & 3.3 & - & - & 7.1 & 19.0 & - & - & 3.6 & - & - & - \\
\hline phyllum & - & 11.1 & - & - & 16.7 & - & - & 19.0 & 14.3 & - & - & - & - & 11.8 & - \\
\hline Potentilla sterilis & 5.4 & - & - & - & 26.7 & - & - & 16.7 & - & - & - & - & - & - & - \\
\hline \multicolumn{16}{|l|}{ Characteristics } \\
\hline Athyrium filix-femina & 6 & 100 & 94.3 & 90.0 & 96.7 & 84.6 & 80.0 & 97.6 & 71.4 & 100 & - & 7.3 & 6.3 & - & 9.1 \\
\hline Blechnuı & 2 & 77.8 & 91.4 & 95.0 & 80.0 & 84.6 & 100 & 64.3 & 42.9 & - & 100 & 30.9 & 43.8 & - & 36.4 \\
\hline n palustre & & 55.6 & 20.0 & 17.5 & 63.3 & 76.9 & 80.0 & 59.5 & 42.9 & - & - & 1.8 & 0.0 & - & - \\
\hline Viola palustris & 83.8 & 44.4 & 97.1 & 70.0 & 26.7 & 46.2 & - & 50.0 & 9.5 & - & 40.0 & 5.5 & - & - & - \\
\hline Carex reuteriana & 54.1 & 100 & 37.1 & 57.5 & 10.0 & - & - & 52.4 & 4.8 & - & - & - & - & - & - \\
\hline Ranunculus ficaria & 32.4 & - & 22.9 & 5.0 & 3.3 & 15.4 & - & 64.3 & 19.0 & - & - & - & - & - & - \\
\hline Brachypodium sylvaticum & 2.7 & 22.2 & - & 5.0 & 3.3 & - & - & 7.1 & - & - & 40.0 & - & - & - & - \\
\hline \multicolumn{16}{|c|}{ Characteristics of Fagetalia sylvaticae } \\
\hline Primula acaulis & 54.1 & 33.3 & 5.7 & 15.0 & 36.7 & 7.7 & 20.0 & 69.0 & 28.6 & - & 40.0 & 1.8 & - & 5.9 & - \\
\hline Ranunculus tuber & 2.7 & - & 5.7 & 12.5 & 16.7 & 23.1 & 60.0 & 9.5 & - & - & - & 5.5 & - & 11.8 & - \\
\hline Mercurialis perennis & 2.7 & - & - & 5.0 & 26.7 & 7.7 & 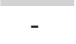 & 2. & - & - & - & 3.6 & - & 11.8 & - \\
\hline \multicolumn{16}{|c|}{ Characteristics of Quercetalia roboris } \\
\hline Teucrium scorodonia & 75.7 & 66.7 & 85.7 & 85.0 & 30.0 & 30.8 & 20.0 & 52.4 & 38.1 & - & 60.0 & 23.6 & 37.5 & 94.1 & 81.8 \\
\hline Holcus mollis & 89.2 & 33.3 & 71.4 & 92.5 & 86.7 & 46.2 & - & 50.0 & 28.6 & - & - & 12.7 & 100 & 94.1 & 81.8 \\
\hline Luzula sylvatica & 70.3 & 66.7 & 14.3 & 35.0 & 96.7 & 76.9 & 100 & 64.3 & 66.7 & 50.0 & 20.0 & 83.6 & - & - & - \\
\hline Vaccinium & 18.9 & - & 22.9 & 50.0 & 70.0 & 100 & 100 & 47.6 & 66.7 & 100 & 100 & 98.2 & 25.0 & 23.5 & - \\
\hline Saxifraga spathularis & 35.1 & 33.3 & 20.0 & 62.5 & 70.0 & 69.2 & 80.0 & 33.3 & 28.6 & - & 60.0 & 70.9 & - & 5.9 & - \\
\hline Dryopteris dilatata & 16.2 & - & 40.0 & 52.5 & 63.3 & 76.9 & 40.0 & 16.7 & 9.5 & 16.7 & 20.0 & 54.5 & 25.0 & 5.9 & - \\
\hline Deschampsia flexuosa & 16.2 & - & 22.9 & 45.0 & 43.3 & 53.8 & - & 16.7 & 14.3 & - & - & 58.2 & 6.3 & 11.8 & - \\
\hline Veronica officinalis & 18.9 & - & 2.9 & - & 10.0 & 30.8 & - & 19.0 & 33.3 & 16.7 & 40.0 & 7.3 & - & - & - \\
\hline Lathyrus linifolius & 18.9 & 77.8 & 8.6 & 2.5 & 13.3 & 7.7 & - & 33.3 & 14.3 & - & - & - & - & - & - \\
\hline Physospermum cornubiense & 8.1 & - & 2.9 & 5.0 & 10.0 & 23.1 & - & 16.7 & - & - & 60.0 & 3.6 & - & - & - \\
\hline
\end{tabular}




\begin{tabular}{|c|c|c|c|c|c|c|c|c|c|c|c|c|c|c|c|}
\hline \multicolumn{16}{|c|}{ Characteristics of Quercetea robori-petraeae and Carpino-Fagetea } \\
\hline Viola riviniana & 54.1 & 22.2 & 51.4 & 25.0 & 50.0 & 38.5 & - & 78.6 & 57.1 & - & 60.0 & 10.9 & 12.5 & 70.6 & 45.5 \\
\hline Dryopteris affinis & 67.6 & 77.8 & 82.9 & 80.0 & 56.7 & 38.5 & - & 45.2 & 47.6 & - & 60.0 & 12.7 & 25.0 & 5.9 & 27.3 \\
\hline Hedera hibernica & 48.6 & 44.4 & 68.6 & 85.0 & 40.0 & 38.5 & - & 50.0 & - & - & 40.0 & 10.9 & 43.8 & 35.3 & 90.9 \\
\hline Crepis lampsanoides & 70.3 & 66.7 & 28.6 & 17.5 & 80.0 & 76.9 & 20.0 & 73.8 & 71.4 & - & 40.0 & 52.7 & - & 17.6 & - \\
\hline Dryopteris filix-mas & 21.6 & - & 2.9 & - & 43.3 & 30.8 & 100 & 50.0 & 33.3 & 33.3 & - & 30.9 & 12.5 & 11.8 & 9.1 \\
\hline Stellaria holostea & 40.5 & 22.2 & 48.6 & 37.5 & 50.0 & 76.9 & - & 52.4 & 42.9 & - & - & 76.4 & 18.8 & 70.6 & 9.1 \\
\hline Euphorbia dulcis & 97.3 & 88.9 & 82.9 & 60.0 & 63.3 & 76.9 & 100 & 66.7 & - & - & 20.0 & 10.9 & - & 17.6 & - \\
\hline Polypodium vulgare & 18.9 & 33.3 & 40.0 & 52.5 & 26.7 & 23.1 & - & 38.1 & 28.6 & - & - & 14.5 & - & 5.9 & - \\
\hline Euphorbia amygdaloides & 10.8 & - & 14.3 & 15.0 & 26.7 & 7.7 & - & 16.7 & 4.8 & - & 20.0 & 14.5 & - & 5.9 & - \\
\hline Oxalis acetosella & 10.8 & - & 22.9 & 37.5 & 86.7 & 84.6 & 100 & 33.3 & 33.3 & - & - & 45.5 & - & 5.9 & - \\
\hline Lonicera hispanica & 75.7 & 77.8 & 77.1 & 75.0 & 43.3 & 38.5 & - & 66.7 & 28.6 & 100 & - & - & - & - & - \\
\hline Anemone nemorosa & 24.3 & 11.1 & 11.4 & 7.5 & 70.0 & 76.9 & - & 40.5 & - & - & - & 60.0 & - & 11.8 & - \\
\hline Hyacinthoides non-scripta & 21.6 & - & 11.4 & 5.0 & 13.3 & 30.8 & 20.0 & 23.8 & - & - & - & 7.3 & - & 35.3 & - \\
\hline Hieracium gr. murorum & 24.3 & - & 2.9 & - & 23.3 & 15.4 & - & 31.0 & 4.8 & - & - & 3.6 & - & - & - \\
\hline Lonicera periclymenum & - & - & - & - & - & 7.7 & 20.0 & - & - & - & 60.0 & 14.5 & 56.3 & 70.6 & 81.8 \\
\hline Ajuga reptans & 32.4 & - & 28.6 & 50.0 & 13.3 & - & - & 50.0 & - & - & - & - & - & 5.9 & - \\
\hline Solidago virgaurea & 8.1 & - & - & 5.0 & 3.3 & 7.7 & - & - & - & - & - & 9.1 & - & - & - \\
\hline \multicolumn{16}{|l|}{ Other species } \\
\hline Pteridium aquilinum & 56.8 & 11.1 & 62.9 & 57.5 & 40.0 & 46.2 & 40.0 & 21.4 & 23.8 & 66.7 & 40.0 & 40.0 & 100 & 100 & 54.5 \\
\hline Omphalodes nitida & 78.4 & 88.9 & 51.4 & 35.0 & 53.3 & 69.2 & 80.0 & 50.0 & 28.6 & - & 60.0 & 10.9 & 12.5 & 17.6 & 9.1 \\
\hline Potentilla erecta & 37.8 & 22.2 & 20.0 & 5.0 & 13.3 & 23.1 & - & 31.0 & 23.8 & 16.7 & 40.0 & 3.6 & 12.5 & - & 18.2 \\
\hline Digitalis purpurea & 73.0 & 44.4 & 48.6 & 40.0 & 3.3 & 15.4 & - & 14.3 & 4.8 & - & - & 23.6 & 18.8 & 47.1 & - \\
\hline Agrostis capillaris & 62.2 & 22.2 & 57.1 & 45.0 & 20.0 & 23.1 & - & 16.7 & - & - & - & 7.3 & 50.0 & 35.3 & 81.8 \\
\hline Rubus sp. & 97.3 & - & 97.1 & 97.5 & 66.7 & 61.5 & - & 76.2 & 33.3 & - & 80.0 & - & 100 & 100 & 90.9 \\
\hline Anthoxanthum odoratum & 13.5 & - & 17.1 & 2.5 & 43.3 & 30.8 & - & 16.7 & 14.3 & - & - & 21.8 & 6.3 & 11.8 & - \\
\hline Rumex acetosa & 32.4 & 44.4 & 20.0 & 17.5 & 26.7 & 15.4 & - & 38.1 & 14.3 & - & - & 9.1 & - & 5.9 & - \\
\hline Caltha palustris & 37.8 & 22.2 & 28.6 & 17.5 & 53.3 & 46.2 & 40.0 & 52.4 & 23.8 & - & - & 1.8 & - & - & - \\
\hline Lotus pedunculatus & 32.4 & 22.2 & 17.1 & 2.5 & 10.0 & 7.7 & 20.0 & 28.6 & 4.8 & 16.7 & - & - & - & - & - \\
\hline Allium victorialis & 16.2 & - & 5.7 & 22.5 & 26.7 & 53.8 & - & 35.7 & 47.6 & - & 60.0 & 12.7 & - & - & - \\
\hline Angelica major & 37.8 & 11.1 & 45.7 & 42.5 & 36.7 & 23.1 & - & 45.2 & 47.6 & - & - & 7.3 & - & - & - \\
\hline Lamium maculatum & 5.4 & - & 17.1 & 22.5 & 10.0 & 15.4 & - & 26.2 & 19.0 & - & - & 7.3 & - & 5.9 & - \\
\hline Centaurea nigra & 21.6 & 33.3 & 51.4 & 45.0 & 10.0 & 23.1 & - & 26.2 & - & - & 40.0 & 1.8 & - & - & - \\
\hline Brachypodium rupestre & 78.4 & 33.3 & 71.4 & 55.0 & 16.7 & 7.7 & - & 64.3 & - & - & - & - & - & 17.6 & 18.2 \\
\hline Deschampsia subtriflora & 73.0 & 33.3 & 71.4 & 87.5 & 6.7 & 46.2 & - & 23.8 & - & - & - & - & 6.3 & - & 27.3 \\
\hline Heracleum sphondylium & 51.4 & 33.3 & 8.6 & 12.5 & 26.7 & 7.7 & 20.0 & 47.6 & 4.8 & - & - & - & - & - & - \\
\hline Juncus effusus & 13.5 & 22.2 & 25.7 & 22.5 & 6.7 & 15.4 & - & 14.3 & 9.5 & 33.3 & - & - & - & - & - \\
\hline Carex laevigata & 59.5 & 33.3 & 45.7 & 50.0 & 20.0 & 23.1 & 20.0 & 21.4 & 4.8 & - & - & - & - & - & - \\
\hline Dactylis glomerata & 64.9 & 44.4 & 60.0 & 47.5 & 26.7 & - & - & 42.9 & - & - & - & 1.8 & 6.3 & 29.4 & - \\
\hline Festuca gr. rubra & 51.4 & - & 11.4 & - & 33.3 & 23.1 & - & 35.7 & - & - & - & 10.9 & 6.3 & 23.5 & - \\
\hline Carex pilulifera & 8.1 & - & 14.3 & 2.5 & 6.7 & - & - & 4.8 & - & - & - & 5.5 & 12.5 & - & 18.2 \\
\hline Veronica chamaedrys & 16.2 & - & 5.7 & 15.0 & 13.3 & - & - & 33.3 & 19.0 & - & - & 3.6 & - & 17.6 & - \\
\hline Ranunculus repens & 45.9 & 11.1 & 48.6 & 30.0 & 53.3 & 46.2 & - & 45.2 & 33.3 & - & - & - & - & - & - \\
\hline Carex echinata & 2.7 & - & 2.9 & 5.0 & 3.3 & 7.7 & - & 2.4 & 4.8 & 100 & - & - & - & - & - \\
\hline Galium saxatile & 2.7 & - & 2.9 & - & 6.7 & 15.4 & 20.0 & - & - & - & - & 25.5 & - & 11.8 & - \\
\hline Lastrea limbosperma & 2.7 & - & 14.3 & 17.5 & 76.7 & 76.9 & - & 2.4 & - & - & - & 5.5 & - & - & - \\
\hline Geranium robertianum & 24.3 & - & 11.4 & - & 33.3 & 23.1 & - & 31.0 & 19.0 & - & - & 3.6 & - & - & - \\
\hline Arrhenatherum bulbosum & 37.8 & 66.7 & 28.6 & 52.5 & 6.7 & - & - & 19.0 & - & - & - & 3.6 & - & - & - \\
\hline Pseudarrhenatherum longif. & 8.1 & 11.1 & 2.9 & 2.5 & - & - & - & - & - & - & - & 1.8 & 6.3 & - & 18.2 \\
\hline Peucedanum lancifolium & 21.6 & 11.1 & 20.0 & 15.0 & 6.7 & 7.7 & - & 21.4 & - & - & - & - & - & - & - \\
\hline Saxifraga lepismigena & 8.1 & 22.2 & 5.7 & 12.5 & 43.3 & 53.8 & - & 2.4 & - & - & - & - & - & - & - \\
\hline Sphagnum auriculatum & 35.1 & 22.2 & 8.6 & 7.5 & 16.7 & - & - & 9.5 & - & 33.3 & - & - & - & - & - \\
\hline Ulex galli & - & - & 5.7 & 7.5 & 3.3 & - & - & 2.4 & - & - & - & - & 6.3 & 5.9 & 27.3 \\
\hline Avenula sulcata & - & - & 5.7 & 10.0 & 3.3 & 7.7 & - & 2.4 & - & - & - & - & 6.3 & 5.9 & - \\
\hline Chrysosplenium oppositif. & 13.5 & - & 8.6 & 2.5 & 33.3 & 38.5 & - & 21.4 & 23.8 & - & - & - & - & - & - \\
\hline Wahlenbergia hederacea & 32.4 & - & 8.6 & - & 6.7 & 7.7 & - & 9.5 & 14.3 & - & - & - & - & - & - \\
\hline Epilobium obscurum & 21.6 & 22.2 & 11.4 & 5.0 & 13.3 & - & - & 11.9 & - & - & - & - & - & - & - \\
\hline Urtica dioica & 5.4 & - & 5.7 & 2.5 & 13.3 & - & - & 19.0 & 19.0 & - & - & - & - & - & - \\
\hline Holcus lanatus & 10.8 & - & 25.7 & - & 6.7 & - & - & 9.5 & 4.8 & - & - & - & - & - & 18.2 \\
\hline Succisa pratensis & 8.1 & - & 2.9 & 7.5 & - & 7.7 & - & 2.4 & - & 50.0 & - & - & - & - & - \\
\hline Prunella grandiflora & 5.4 & 22.2 & 2.9 & 5.0 & 6.7 & - & - & 4.8 & - & - & - & - & - & - & - \\
\hline Galium aparine & 5.4 & - & 5.7 & - & 3.3 & - & - & 26.2 & - & - & - & 1.8 & - & 5.9 & - \\
\hline Vicia sepium & 13.5 & 22.2 & - & 2.5 & 20.0 & - & - & 9.5 & 14.3 & - & - & - & - & - & - \\
\hline Prunella vulgaris & 29.7 & - & 25.7 & 22.5 & 13.3 & - & - & 11.9 & 9.5 & - & - & - & - & - & - \\
\hline Luzula multiflora & 8.1 & 11.1 & - & - & - & 7.7 & - & 9.5 & 4.8 & - & - & - & - & - & - \\
\hline
\end{tabular}




\begin{tabular}{|c|c|c|c|c|c|c|c|c|c|c|c|c|c|c|c|}
\hline & 2.7 & - & & 5.0 & 10.0 & 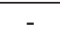 & - & 23.8 & 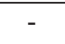 & 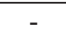 & & - & & - & \\
\hline m commune & 10.8 & - & 14.3 & 15.0 & 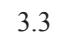 & - & 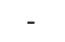 & 4 & 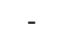 & - & & - & & - & \\
\hline ta & 7 & - & .3 & 2.5 & .7 & - & - & 1.3 & - & - & & - & - & - & \\
\hline Ilob & 2.7 & - & 8.6 & - & - & & .0 & .1 & - & - & & - & & - & \\
\hline mpervirens & 5.4 & 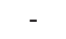 & - & 2.5 & 10.0 & 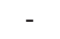 & & 1.9 & 8 & - & & 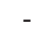 & & - & \\
\hline Im & 10.8 & - & - & - & 3.3 & & & & & 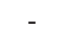 & & - & & - & \\
\hline & - & - & - & 2.5 & 3.3 & & - & 1.9 & . & 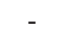 & & 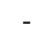 & & - & \\
\hline & - & - & - & - & 13.3 & - & - & - & & & & & & 8 & \\
\hline is & - & & - & 2.5 & - & & - & 21.4 & - & - & & & & - & \\
\hline \multicolumn{16}{|c|}{$\begin{array}{l}\text { Sources of relevés: Cr-Bc: atlantic-orolusitanian and galician-portugueses riparian birch forests: } 1 \text { (1a): Table 2; } 2 \text { (1b): Honra } \\
\text { et al. (2003), Table 1: } 9 \text { rel. Vp-Bp: N galician-asturian riparian birch-forests: } 3 \text { (2a): Table 3; } 4 \text { (2b): Rodríguez Guitián, M. } \\
\text { (2010): Table 6: } 40 \text { rel. Ch-Bp: western orocantabrian riparian birch-forests: } 5 \text { (3a): Table 4; } 6 \text { (3b): Silva-Pando (2009), Tab } \\
\text { 3: rel. 3, 4, 12; Rodríguez-Guitián et al. (2013), Table 10: rel. 1, 2; Rodríguez Guitián et al. (2014), Table 10: rel. 9-16. Lh-Bcs } \\
\text { supratemperate western orocantabrian birch forests (hygrophilous): } 7 \text { (4): Izco et al. (986), Table 4: rel. 8-12. Vo-Bp: galicia } \\
\text { duriensian riparian birch-forests: } 8 \text { (5a): Table 5; } 9 \text { (5b): Gonçalves Aguiar (2000), Table 125: } 2 \text { rel.; Rodríguez Guitián } \\
\text { Bariego Hernández (2009), Table 1: rel. 1-5, 7-9, 12, 13, 15; González de Paz (2012), Table 6.117: } 8 \text { rel. Rc-Savm: Monca } \\
\text { riparian birch forests: } 10 \text { (6): Navarro (1989): Table 10: } 6 \text { rel. Ej-Bc: supratemperate atlantic-orolusitanian birch forests: } \\
\text { (7): Costa et al. (2012), rel. page 86; Pulgar (1990), Table 4: } 4 \text { rel. Lh-Bct: supratemperate western orocantabrian birch fore } \\
\text { (typical); } 12 \text { (8): Izco et al. (1986), Table 3: rel. 1-7; Ortiz (1986), Table 91: } 6 \text { rel.; Fernández Prieto \& Vázquez (1987), Tab } \\
\text { 4: } 9 \text { rel.; Puente García (1988), Table 72: } 10 \text { rel.; Costa et al. (1990), Table 1: } 4 \text { rel.; Romero Rodríguez \& Romero Cuen } \\
\text { (1996), Table 10: rel. 1, 3; Rodríguez Guitián et al. (2000), Table 1: rel. 1; Romero Rodríguez \& Romero Cuenca (2004), Tab } \\
\text { 4: } 4 \text { rel.; Silva-Pando (2009), Table 1: } 10 \text { rel. Hm-Bct: seral galician-asturian and galician-portuguese birch forests (typical): } \\
\text { (9a): Amigo \& Romero (1998): Table 1: } 16 \text { rel. Hm-Bcm: seral western orocantabrian birch forests ("montane"): 14 (9b): Ami } \\
\text { (1984), Table 12: } 9 \text { rel.; Amigo \& Romero (1998), Table 3: rel. 1-5; Rodríguez Guitián et al. (2000), Table 1: rel. 8; Rodrígue } \\
\text { Guitián et al. (2013), Table 3: rel. 5, 6. Hm-Bch: seral galician-asturian and galician-portuguese birch forests (hygrophilous): } \\
\text { (9c): Amigo \& Romero (1998): Table 2: } 11 \text { rel. }\end{array}$} \\
\hline
\end{tabular}

The existence of a pair of Ch-Bp relevés at the bottom of Figure 3, collected in the eastern extreme of the River Sil headwaters, near to several Vo-Bp relevés collected in the Sanabria Mountains, suggests a floristic relationship between these two areas, but a more detailed sampling would be needed to confirm it.

These four groups can be related to previously described forests: northern Galician-Asturian birch forests (akin to those in association Violo palustris-Betuletum pubescentis), birch forests of the SW Galician and NW Portuguese ranges (akin to those in association Carici reuterianae-Betuletum celtibericae), birch forests of the SE Galician, NE Portuguese and NW Castile-Leon moutains (akin to those described in Sanabria by Rodríguez Guitián \& Bariego Hernández (2008) and birch forests of the $\mathrm{W}$ extreme of the Cantabrian Range (Courel, Ancares, Muniellos and Laciana mountains), in part identified with the subass. salicetosum atrocinereae of the association Luzulo henriquesii-Betuletum celtibericae by several authors. Table 1 sketches the differences in floristic composition among these four groups and between these and the other non riparian forests discussed in this study. Figure 4 contains a dichotomic key for the floristic discrimination of these forests.
The distinctive floristic feature shared by all these communities was the almost complete absence of thermophilous species, such as Arbutus unedo, Asplenium onopteris, Laurus nobilis, Ruscus aculeatus and Tamus communis, considered as bioclimatic indicators of the termotemperate and, to a lesser extent, the mesotemperate horizon in the territory (cf. Rodríguez Guitián \& Ramil Rego 2007). These species were only found in eight of the 227 relevés. This is consistent with the fact that almost $74 \%$ of the sampled forests were located above an altitude of $700 \mathrm{~m}$, considered as an average value for the lower limit of the supratemperate bioclimatic horizon at a regional level (Rodríguez Guitián \& Ramil Rego, 2007).

The following is a detailed description of the floristic, ecologic and biogeographic characteristics of these communities.

\section{Atlantic-orolusitanian and Galician-portuguese (meso-)supratemperate riparian birch forests (Carici reuterianae-Betuletum celtibericae Hon- rado, P. Alves, Aguiar, Ortiz \& FB Caldas ex Honrado 2004)}

Forest community described by Honrado et al. (2003) and typified later by Honrado (2004), from data collected in higher mesotemperate and supratemperate territories in the $\mathrm{W}$ and $\mathrm{S}$ slopes of the Peneda-Gerês Mountains (N Portugal). 


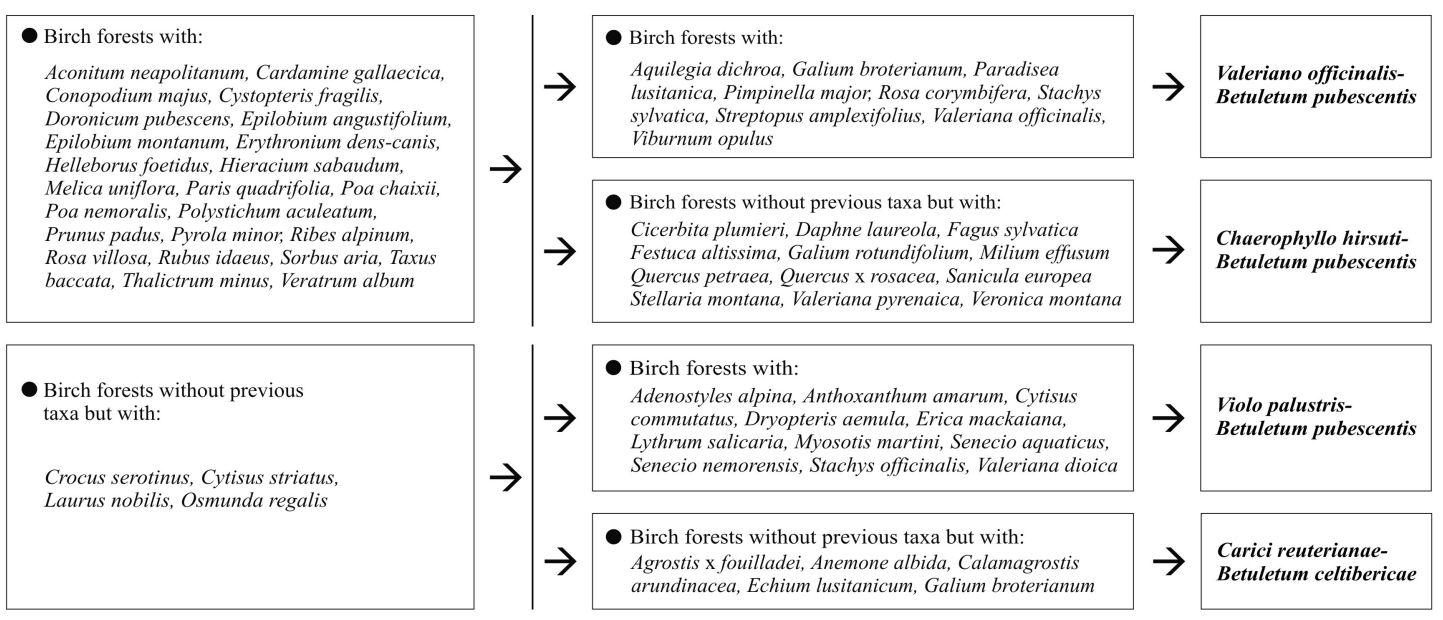

Figure 4. Floristic key to differentiate the riparian birch forests of the NW Iberian Peninsula.

Our data showed that this association is also common in the Spanish slopes of those mountains, between 900 and $1200 \mathrm{~m}$ asl (lower supratemperate horizon), from the vicinity of the Miño River to the Támega River valley (Figures 1 and 5, Table 2). Nevertheless, it was not detected by Pulgar (1999) in his study of natural vegetation of the Baixa Limia-Serra do Xurés Natural Park, a protected area that includes the main part of these mountains, although its presence was implicitly admitted by this author in later studies (Pulgar, 2005; Pulgar \& Manso, 2010).

We have also recognized this community in the headwaters of several rivers in the Central Galician Mountains, between the Ulla and the Miño river valleys. In these cases, the sampling sites were located in lower altitudes, between 500 and $800 \mathrm{~m}$. We attribute this fact to two causes. Firstly, the proximity of these mountains to the coast produces a more oceanic climate and a local descent of the supratemperate horizon to $600 \mathrm{~m}$ (cf. Rodríguez Guitián \& Ramil Rego, 2007). Secondly, the torrential character of many of these rivers prevents the growth of alders (Alnus glutinosa) and ashes (Fraxinus excelsior, F. angustifolia; Figure 3), which dominate the riparian communities at the same altitudes in neighboring, but more inner, areas. The mechanical effect of the running waters on the stability of the riverbanks was also mentioned by Honrado et al. (2003) as a relevant ecological factor in the western and southern slopes of the Peneda-Gerês Mountains, NW Portugal, which receive a considerable amount of annual rainfall, as well. The substrates on which these forests grow are mainly granitic, although in the Cen- tral Galician Mountains they also appear on acid metamorphic rocks (schists, greywackes and quartzites) (IGME, 2017).

In its commonest form (typical variant), these forests are clearly dominated by birches but other tree species are also present, such as Salix atrocinerea, Quercus robur, Frangula alnus, Pyrus cordata and, seldom, Quercus pyrenaica (Figure 5, photos 1a and 1b). In the lower stratum grow Erica arborea and many herbaceous species such as Arrhenatherum bulbosum, Aquilegia dichroa, Athyrium filix-femina, Blechnum spicant, Brachypodium rupestre, Cirsium palustre, Crepis lampsanoides, Dactylis glomerata, Deschampsia cespitosa, Digitalis purpurea, Dryopteris affinis, Euphorbia dulcis, Galium broterianum, Hedera hibernica, Holcus mollis, Lonicera hispanica, Luzula sylvatica, Oenanthe crocata, Omphalodes nitida, Polypodium vulgare, Teucrium scorodonia and Viola palustris. A facies dominated by Salix atrocinerea grows where this community has been disturbed (by felling, for example, to enhance the productivity of hay meadows or arable lands in the riverside) or in stretches of more torrential character with rocky or very unstable substrates (Figure 5, photos 1a and 1b). In places where these forests contact with riparian black alder forests we observed a variant with Alnus glutinosa. A supratemperate variant with Sorbus aucuparia can be found in higher sites.

Distribution: Inner Galician (Ulloa-Deza, Ourensian-Luguese, Penedese and Geresian subsectors) and Galician-Portuguese (Rías Baixas subsector) sectors.

Differential taxa with respect to other riparian birch forests: Anemone trifolia subsp. albida, Echium lusitanicum, Senecio doria. 
Differential taxa with respect to VioloBetuletum: Aquilegia dichroa, Allium scorzonerifolium, Calamagrostis arundinacea, Fraxinus angustifolia, Galium broterianum, Lilium martagon, Paradisea lusitanica, Polygonum bistorta, Ranunculus platanifolius.

Differential taxa with respect to ChaerophylloBetuletum: Allium scorzonerifolium, Aquilegia dichroa, Calamagrostis arundinacea, Fraxinus angustifolia, Galium broterianum, Paradisea lusitanica.

Differential taxa with respect to ValerianoBetuletum: Osmunda regalis.

Note: deeper studies are needed to clarify the relationships between this association and both the Atlantic-Oroportuguese climatophilous birch forests (Eryngio juresiani-Betuletum celtibericae, Costa et al. 2012) and those with higrophilous features described by Pulgar (1999) in the Spanish Serra do Xurés.

\section{Western Cantabrian and Northern Galician- Portuguese meso-supratemperate riparian birch forests (Violo palustris-Betuletum pubescentis Rodríguez Guitián 2010)}

Riparian birch forests described by Rodríguez Guitián (2010) from a set of relevés collected in the headwaters of the rivers flowing into the W extreme of the Cantabric Sea and into the Artabrian Gulf, as well as in the most northern headwaters in the Miño River basin, in lower supratemperate and higher mesotemperate territories. We also observed them in more western and southern areas (Figures 1 and 5, Table 3): in the sources of several tributaries of the rivers Tambre and Miño in the northern half of the Central Galician Mountains to the north of the Ulla River catchment, and in the mountains placed near the boundary of Galicia and NE Asturias and in the middle basin of the Eo River (Figure 5, photos $2 \mathrm{a}$ and $2 \mathrm{~b}$ ). These forests grow on a variety of highly humiferous and acid soils formed from metamorphic rocks (slates, schists, quarzites), granites and detritic cenozoic sediments (IGME, 2017).

These birch forests are characterized by the frequent presence of Salix atrocinerea, Quercus robur, Pyrus cordata and Frangula alnus as companion trees, Lonicera hispanica and Hedera hibernica as the most common vines and Angelica major, Arrhenatherum bulbosum, Athyrium filix-femina, Blechnum spicant, Brachypodium rupestre, Carex laevigata, Centaurea rivularis, Dactylis glomerata, Deschampsia cespitosa, Dryopteris affinis, D. dilatata, Euphorbia dulcis,
Holcus mollis, Oenanthe crocata, Pteridium aquilinum, Rubus sp., Senecio nemorensis, Stellaria holostea, Teucrium scorodonia and Viola palustris, as the most constant herbaceous plants in the understory. As in the previous community, a variant with Alnus glutinosa can be found in stretches were birch and alder forests contact, as well as a Salix atrocinerea facies in anthropized zones close to crop fields or haymeadows (Table 3).

Distribution: Galician-Asturian (Western Cantabrian subsector) and Galician-Portuguese (Chairego subsector) sectors.

Differential taxa with respect to other riparian birch forests: Cytisus commutatus, Dryopteris aemula, Lythrum salicaria, Myosotis martinii, Senecio nemorensis, Stachys officinalis, Valeriana dioica.

Differential taxa with respect to CariciBetuletum: Adenostyles alpina, Narcissus asturiensis, Ranunculus tuberosus, Senecio nemorensis, Silene dioica, Valeriana pyrenaica.

Differential taxa with respect to Chaerophyllo-Betuletum: Cirsium filipendulum, Crocus serotinus, Cytisus striatus, Galium palustre, Molinia caerulea, Oenanthe crocata, Osmunda regalis, Pyrus cordata, Quercus robur, Scrophularia auriculata, Scutellaria minor.

Differential taxa with respect to ValerianoBetuletum: Crocus serotinus, Narcissus asturiensis, Osmunda regalis, Valeriana montana, $\mathrm{Va}$ leriana pyrenaica.

\section{Western orocantabrian supratemperate ri- parian birch forests (Chaerophyllo hirsuti- Betuletum pubescentis ass. nova hoc loco)}

Riparian birch forests growing in supratemperate areas of the headwaters of rivers in the western Cantabrian Range (Navia, Narcea and Sil river basins), on acid metamorphic substrates (slates, schists, quartzites, sandstones) or, occasionally, on granites (Table 4). Birch is usually accompanied by Salix atrocinerea, Sorbus aucuparia, Ilex aquifolium, Quercus petraea or Corylus avellana, and the most constant species at ground level are Adenostyles alpina, Anemone nemorosa, Athyrium filix-femina, Blechnum spicant, Chaerophyllum hirsutum, Cirsium palustre, Crepis lampsanoides, Dryopteris dilatata, Erica arborea, Euphorbia hyberna, Holcus mollis, Lastrea limbosperma, Luzula sylvatica, Omphalodes nitida, Oxalis acetosella, Poa nemoralis, Ranunculus platanifolius, Rubus sp.,Saxifragalepismigena,S. spathularis, Vaccinium myrtillus and Valeriana montana. 


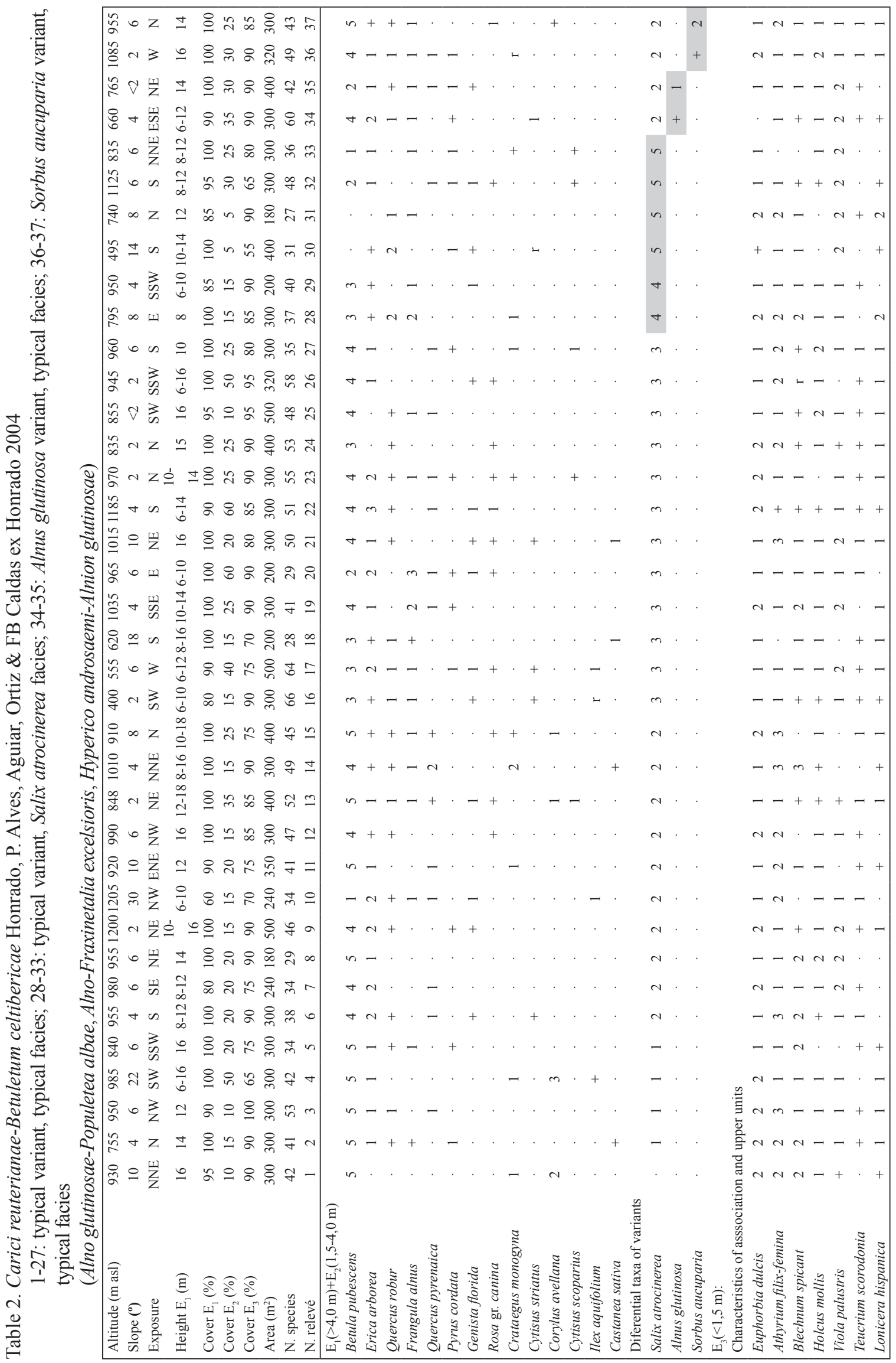




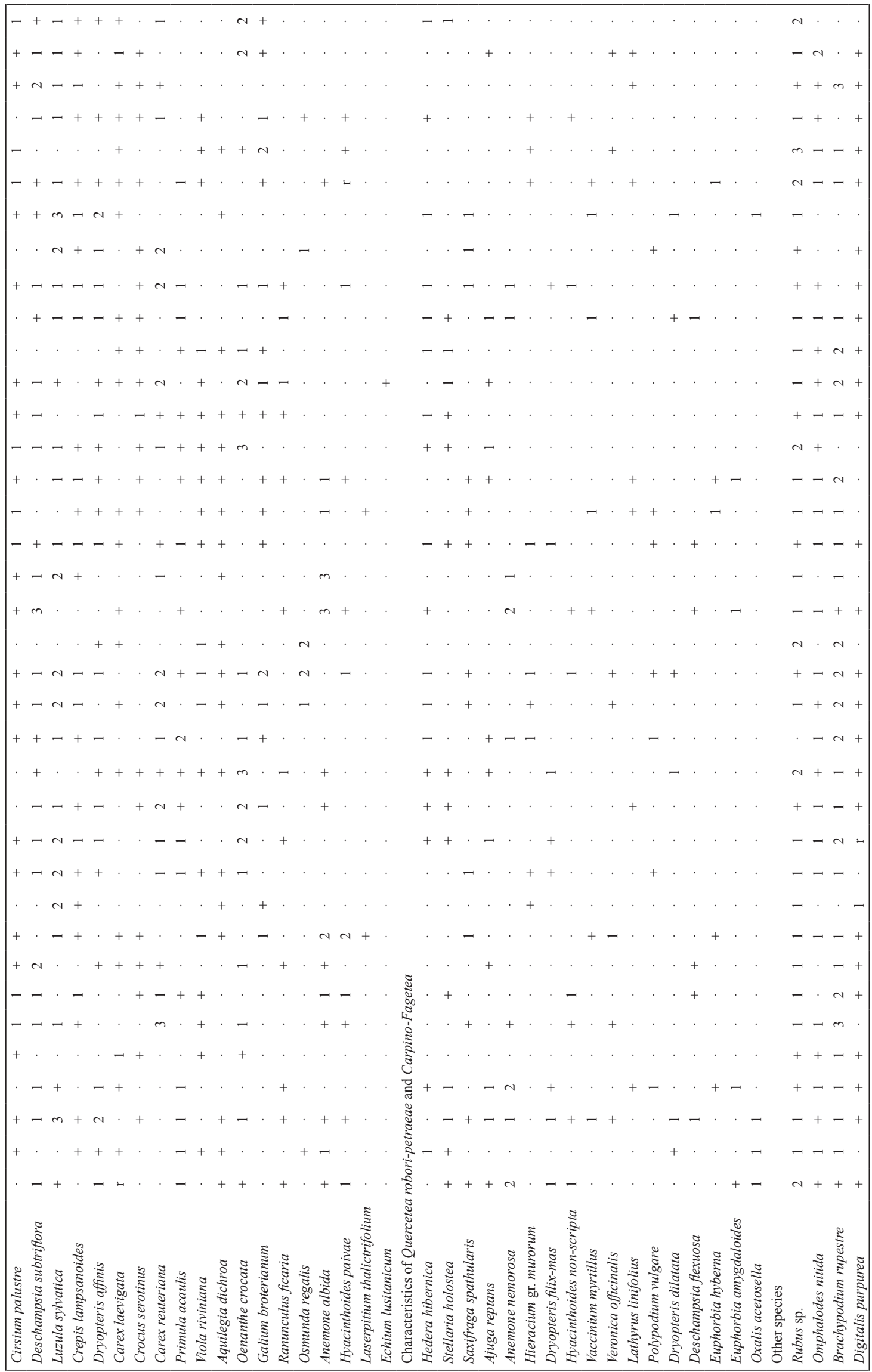




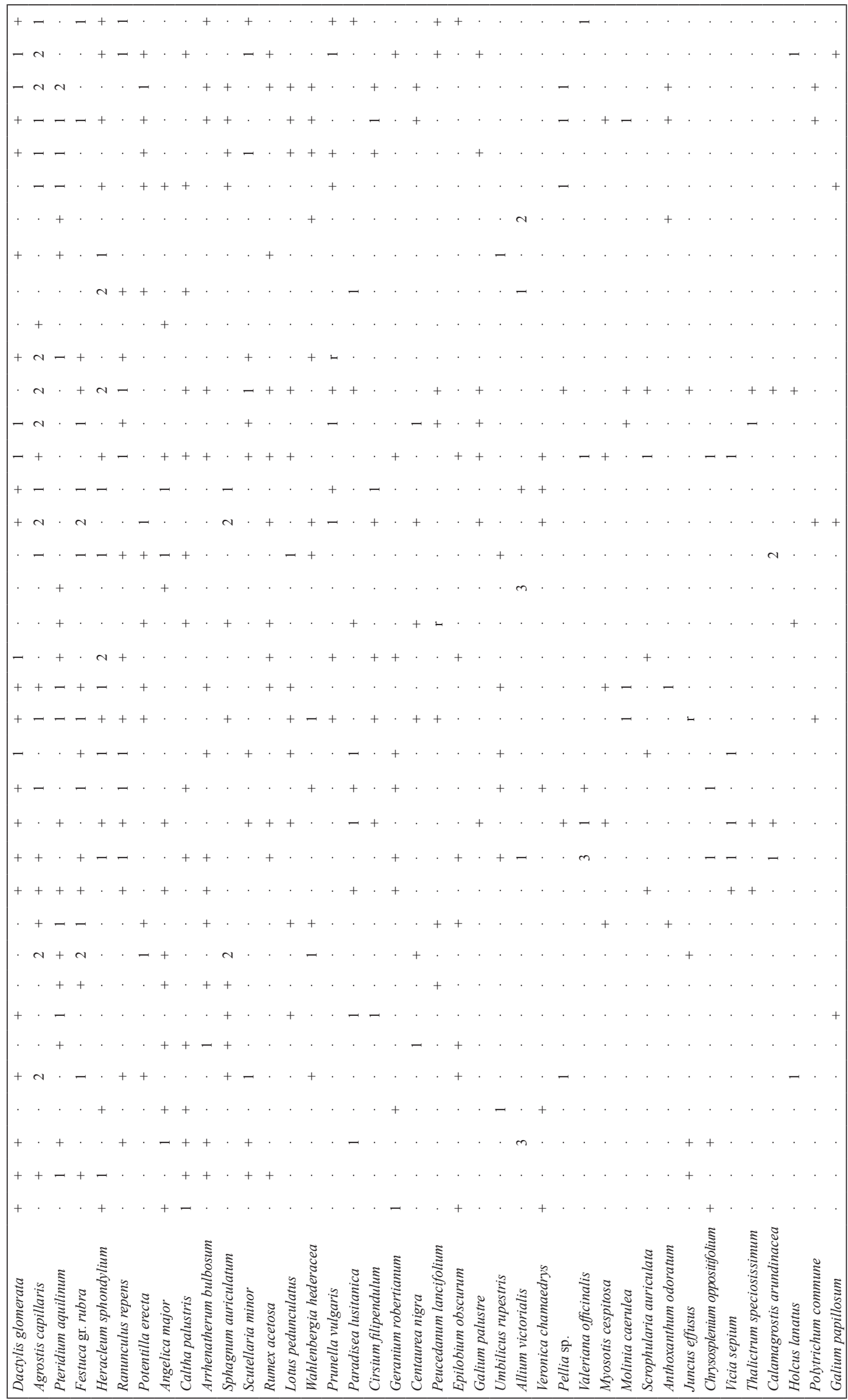




\begin{tabular}{|c|c|}
\hline 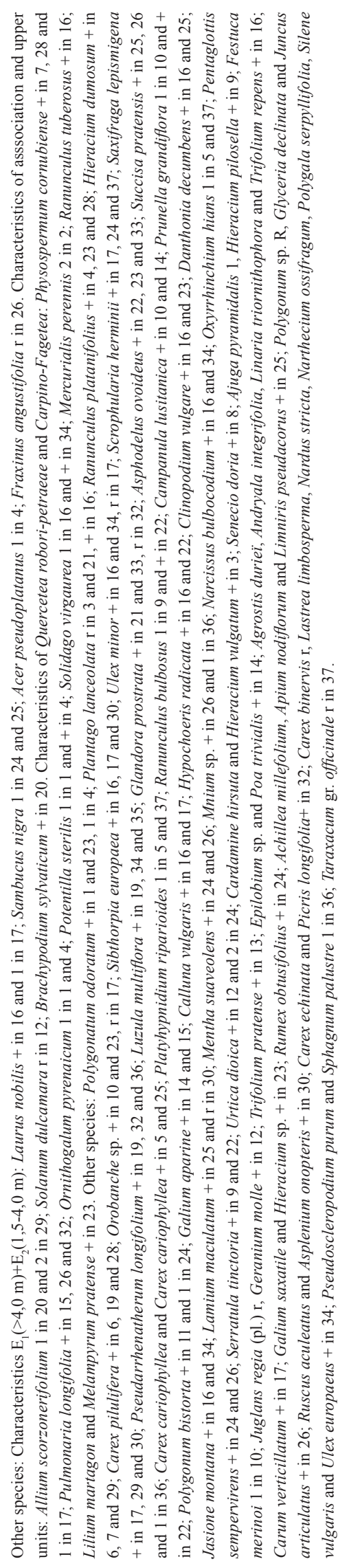 & 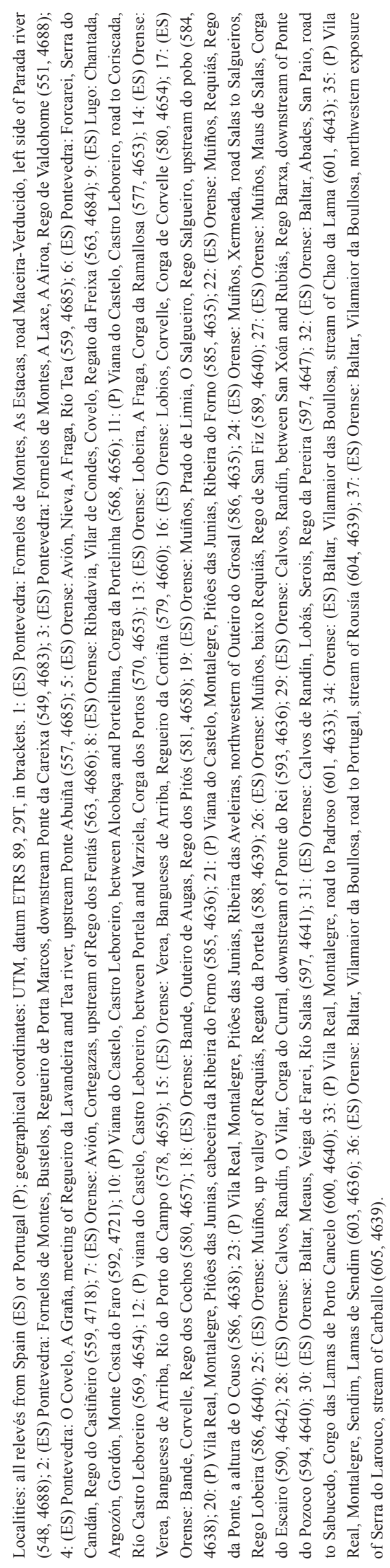 \\
\hline
\end{tabular}



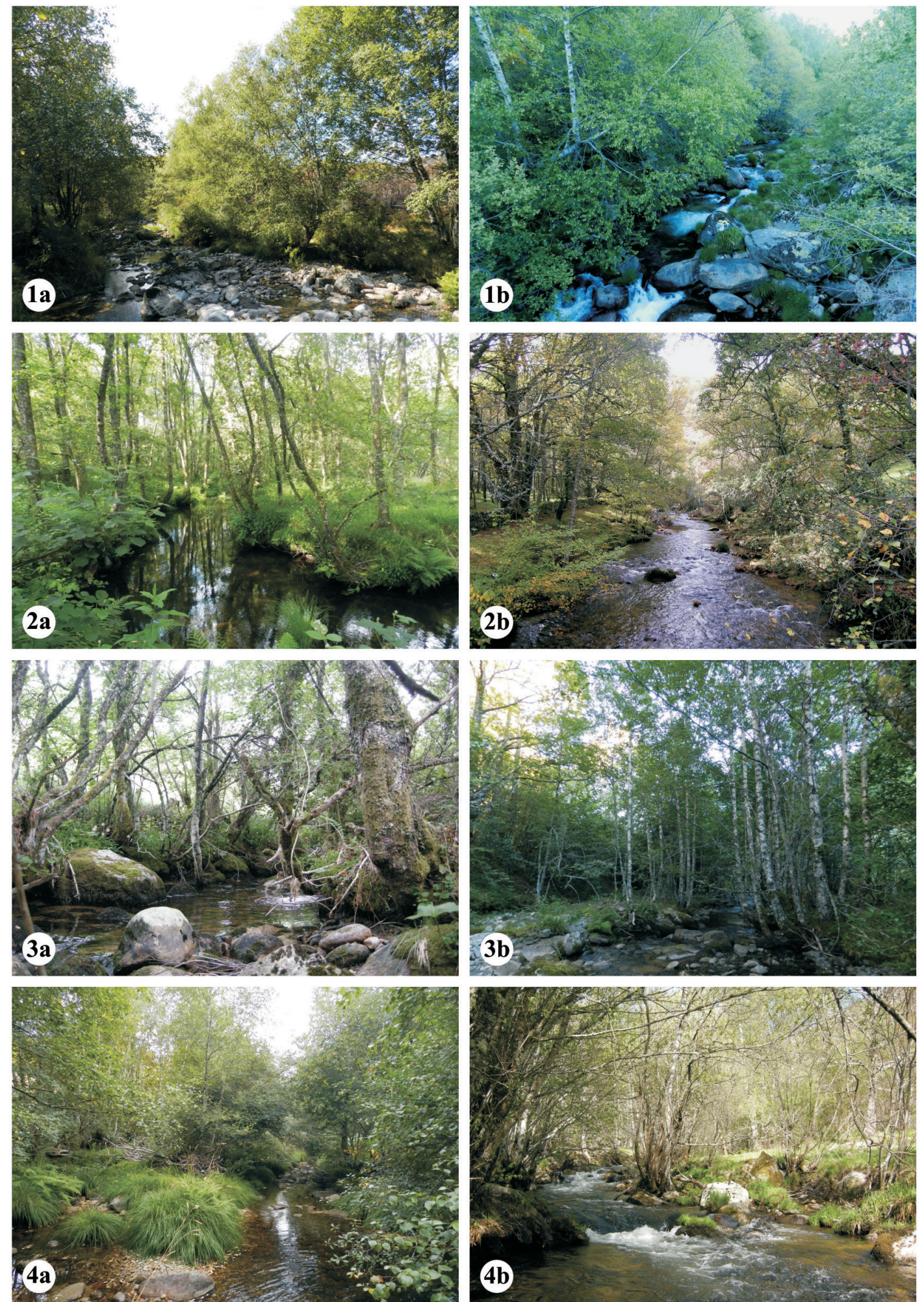

Figure 5. Summer views of some of the riparian birch forests studied. Carici reuterianae-Betuletum celtibericae; 1a: Spain: Pontevedra province, Covelo, A Graña, Regueiro da Lavandeira; 1b: Portugal: Terras de Bouro, Castro Laboreiro, Corga dos Portos. Violo palustris-Betuletum pubescentis; 2a: Spain: Lugo province: Friol, River Parga; 2b: Spain: Lugo province, Muras, River Eume. Chaerophyllo hirsuti-Betuletum pubescentis; 3a: Spain, León province, Candín, Suárbol, Río da Veiga; 3b: Spain: León province, Villafranca del Bierzo, River Porcarizas. Valeriano officinalis-Betuletum pubescentis: 4a: Spain: Ourense province, Chandrexa de Queixa, River Grande; 4b: Spain, Zamora province, Porto, Pías, Río de Barjacova. 
Several authors included this type of forests in the subassociation salicetosum atrocinereae of the association Luzulo henriquesii-Betuletum pubescentis described by Izco et al. (1986), but either extending the original ecological description (Rodríguez Guitián et al. 2013, 2014), or considering them as "hazel-willow forests of the middle and upper supratemperate horizon in humid to hiperhumid ombroclimates on acidic substrates (Luzulo henriquesi-Betuletum celtibericae subass. salicetosum atrocinereae Izco, Amigo \& Guitián 1986 corr. et ampl.)" (Silva-Pando, 2009). We consider both as distorted interpretations of the original subassociation, which was not described explicitly as including riparian forests.

In our opinion, these birch forests constitute a new association because they are always associated to water courses (Figure 5 , photos $3 \mathrm{a}$ and $3 \mathrm{~b}$ ), i.e. they are azonal forests, not exclusive of the upper supratemperate horizon, on the contrary of those of the association Luzulo-Betuletum (cf. Rivas-Martínez et al., 1984; Izco et al., 1986). They are able to reach lower territories, in some cases down to the limit of the lower supratemperate and high mesotemperate horizons, coming into contact with many other forest types such as Quercus petraea and $Q$. pyrenaica forests, holly-tree forests, mixed broadleaved forests and, of course, with the chionophilous birch forests of supratemperate areas. In accordance with their azonality, many hygrophilous vascular plant that can be found in them are missing from the supratemperate birch forests of the ass. LuzuloBetuletum (see groups 3a, 3b and 4, Table $1)$. We therefore propose the inclusion of these riparian birch forests in a new association, named Chaerophyllo hirsuti-Betuletum pubescentis ass. nova hoc loco, for which we choose as holotypus rel. 22, Table 4

The floristic variability of this association allows differentiating a widespread (typical) variant, an Alnus glutinosa variant at the lowest sites as a transition to grey alder-forests, and an upper supratemperate variant with abundant (even dominant) Sorbus aucuparia.
Distribution: Western Orocantabric sector. The presence of this syntaxon in other siliceous areas of the Cantabrian Range must be further investigated.

Differential taxa with respect to other riparian birch forests: Cicerbita plumieri, Daphne laureola,Fagussylvatica, Miliumeffusum, Galium rotundifolium, Quercus petraea, Quercus x rosacea, Sanicula europaea, Scrophularia alpestris.

Differential taxa with respect to CariciBetuletum:Aconitumneapolitanum, Cardamine gallaecica, Doronicum pubescens, Epilobium montanum, Hieracium murorum, Lastrea limbosperma, Mercurialis perennis, Narcissus asturiensis, N. nobilis, Poa chaixii, Poa nemoralis, Polygonatum verticillatum, Polystichum setiferum, Salix caprea, Valeriana montana, Valeriana pyrenaica.

Differential taxawithrespect to Violo-Betuletum and Valeriano-Betuletum: Astrantia major, Cicerbita plumieri, Daphne laureola, Fagus sylvatica, Galium odoratum, G. rotundifolium, Laserpitium merinoi, Milium effusum, Ornithogalumpyrenaicum, Polygonumbistorta, Quercus petraea, Quercus x rosacea, Sanicula europaea, Scrophularia alpestris.

Note: the forests included in this association are somewhat similar to the hygrophilous birch forests of the subass. salicetosum atrocinereae of the Luzulo-Betuletum described by Izco et al. (1986), but several taxa such as Allium victorialis, Angelica major, Aquilegia vulgaris, Cardamine gallaecica, Carex remota, Chrysosplenium oppositifolium, Deschampsia subtriflora, Lastrea limbosperma, Polystichum setiferum, Ranunculus repens, Saxifraga lepismigena, Silene dioica or Viola palustris were not present in their relevés and can be employed to differentiate between these two sintaxa. On the other hand, some of the published relevés of the typical subass. of the orocantabrian riparian birch forests (Luzulo henriquesii-Betuletum celtibericae) could belong to subass. salicetosum atrocinereae or even to the riparian birch forests of ass. ChaerophylloBetuletum, but the lack of detailed environmental and geographical information in many of the studies reviewed prevents the assessment of their status. 
Table 3. Violo palustris-Betuletum pubescentis Rodríguez Guitián 2010 typical variant, typical facies

(Alno glutinosae-Populetea albae, Alno-Fraxinetalia excelsioris, Hyperico androsaemi-Alnion glutinosae)

\begin{tabular}{|c|c|c|c|c|c|c|c|c|c|c|c|c|c|c|c|c|c|c|c|c|c|c|c|c|}
\hline Altitude ( $\mathrm{m}$ asl) & 725 & 625 & 515 & 570 & 475 & 540 & 440 & 435 & 510 & 445 & 470 & 475 & 520 & 500 & 595 & 740 & 455 & 515 & 495 & 450 & 420 & 460 & 605 & 675 \\
\hline Slope $\left({ }^{\circ}\right)$ & 10 & $<2$ & 4 & 2 & $<2$ & 2 & $<2$ & $<2$ & 4 & 4 & $<2$ & 2 & $<2$ & 2 & 2 & 18 & 6 & 4 & 4 & 8 & $<2$ & 2 & 2 & 18 \\
\hline Exposition & SSE & NW & $\mathrm{E}$ & $\mathrm{N}$ & SE & $\mathrm{S}$ & $\mathrm{E}$ & ENE & SW & NW & NNE & SSE & SSE & $\mathrm{N}$ & ENE & $\mathrm{N}$ & SSW & SSW & $V \mathrm{E}$ & ENE & SSE & $\mathrm{E}$ & NNE & $\mathrm{EW}$ \\
\hline Height (m) & 14 & 18 & $6-14$ & 16 & 17 & 13 & 20 & 20 & $8-12$ & 16 & 20 & 18 & 16 & 14 & 16 & $8-16$ & 14 & 16 & 14 & $10-14$ & 420 & 18 & 16 & 14 \\
\hline Cover $E_{1}(\%)$ & 100 & 100 & 100 & 95 & 100 & 95 & 100 & 100 & 100 & 100 & 100 & 90 & 95 & 100 & 100 & 90 & 90 & 95 & 90 & 100 & 100 & 90 & 100 & 100 \\
\hline Cover $\mathrm{E}_{2}(\%)$ & $<5$ & 25 & 30 & 25 & 20 & 30 & 15 & 30 & 20 & 25 & 30 & 20 & 20 & 35 & 40 & 20 & 20 & 25 & 40 & 15 & 20 & 35 & 50 & 15 \\
\hline Cover $\mathrm{E}_{3}(\%)$ & 90 & 90 & 95 & 90 & 100 & 85 & 100 & 95 & 85 & 95 & 90 & 90 & 100 & 100 & 100 & 95 & 95 & 75 & 90 & 75 & 100 & 90 & 100 & 85 \\
\hline Area $\left(\mathrm{m}^{2}\right)$ & 300 & 280 & 300 & 400 & 300 & 200 & 300 & 200 & 320 & 400 & 200 & 200 & 200 & 200 & 300 & 300 & 400 & 300 & 200 & 100 & 200 & 120 & 200 & 300 \\
\hline N. species & 30 & 25 & 30 & 53 & 24 & 29 & 37 & 43 & 34 & 45 & 39 & 42 & 35 & 28 & 47 & 39 & 33 & 43 & 25 & 27 & 33 & 36 & 29 & 51 \\
\hline N. relevé & 1 & 2 & 3 & 4 & 5 & 6 & 7 & 8 & 9 & 10 & 11 & 12 & 13 & 14 & 15 & 16 & 17 & 18 & 19 & 20 & 21 & 22 & 23 & 24 \\
\hline \multicolumn{25}{|l|}{$\mathrm{E}_{1}(>4,0 \mathrm{~m})+\mathrm{E}_{2}(1,5-4,0 \mathrm{~m})$} \\
\hline Betula pubescens & 5 & 5 & 5 & 5 & 5 & 5 & 5 & 5 & 4 & 4 & 4 & 4 & 4 & 4 & 4 & 4 & 4 & 4 & 4 & 4 & 4 & 4 & 3 & 3 \\
\hline Salix atrocinerea & 1 & 1 & 1 & 1 & 1 & 1 & 1 & 2 & + & 1 & 1 & 1 & 1 & 1 & 1 & 2 & 2 & 2 & 2 & 2 & 2 & 2 & . & 3 \\
\hline Quercus robur & 1 & 1 & 2 & 1 & 1 & 1 & 1 & 1 & 2 & 3 & 2 & 2 & 2 & 2 & 1 & 2 & 1 & 1 & . & + & 2 & 1 & 3 & 2 \\
\hline Frangula alnus & . & 1 & . & 1 & 1 & . & 1 & 1 & 2 & 2 & 1 & + & 2 & . & 1 & . & 1 & 1 & 2 & 1 & + & 1 & 3 & + \\
\hline Erica arborea & . & 2 & + & 1 & 1 & 1 & 2 & 1 & 1 & 1 & 1 & 1 & + & . & 1 & . & 1 & 1 & 1 & . & 2 & + & 1 & + \\
\hline Pyrus cordata & . & · & . & + & . & . & . & . & 1 & 1 & . & . & 1 & . & + & · & 1 & + & . & . & 1 & 1 & 1 & 1 \\
\hline Ilex aquifolium & . & . & + & $\mathrm{r}$ & . & . & . & . & + & 1 & . & . & . & . & . & 1 & 1 & + & . & . & . & . & . & + \\
\hline Corylus avellana & . & . & . & 1 & . & . & . & . & . & . & . & + & . & . & . & 2 & . & 2 & . & . & . & . & . & 1 \\
\hline Castanea sativa & . & 2 & 1 & . & . & + & . & . & . & $\mathrm{r}$ & . & . & . & . & . & . & 1 & . & . & . & . & . & . & . \\
\hline Sambucus nigra & . & . & 1 & . & . & . & . & + & . & . & 1 & 1 & . & . & + & . & . & . & . & . & . & . & . & . \\
\hline Cytisus scoparius & . & + & . & . & . & . & . & . & . & . & + & . & . & + & . & . & 1 & . & . & . & . & . & . & . \\
\hline Acer pseudoplatanus & · & $\cdot$ & . & $\mathrm{r}$ & . & 1 & . & . & · & · & . & . & · & · & $\cdot$ & 1 & . & $\mathrm{r}$ & . & . & . & . & . & . \\
\hline Quercus pyrenaica & . & . & . & . & . & . & . & . & . & . & . & . & . & . & . & . & $\cdot$ & . & . & . & 2 & . & 3 & . \\
\hline Fraxinus excelsior & . & · & 1 & 1 & . & . & . & · & · & · & · & . & . & . & . & . & . & . & . & . & · & · & · & . \\
\hline Sorbus aucuparia & . & . & . & . & . & . & . & . & · & . & . & . & . & . & . & 1 & · & . & . & · & . & . & . & + \\
\hline Genista florida & . & + & . & . & . & . & . & . & . & . & . & . & . & . & $\mathrm{r}$ & . & · & . & . & · & . & . & . & · \\
\hline Prunus avium & 1 & · & . & . & . & . & . & . & . & . & . & . & . & . & . & . & . & . & . & . & . & . & . & . \\
\hline Ligustrum ovalifolium & . & . & . & . & . & 1 & . & . & . & . & . & . & . & . & . & . & . & . & . & . & . & . & . & . \\
\hline Rosa gr. canina & . & . & . & + & . & . & . & . & . & . & . & . & . & . & . & . & . & . & . & . & . & . & . & . \\
\hline Cytisus striatus & . & . & . & . & . & . & . & . & . & + & . & . & . & . & . & . & . & . & . & . & . & . & . & . \\
\hline \multicolumn{25}{|c|}{ Characteristcs of association and upper units $\mathrm{E}_{3}(<1,5 \mathrm{~m})$} \\
\hline Viola palustris & + & 1 & 1 & 1 & 1 & 2 & 2 & 2 & 2 & 2 & 1 & 2 & 2 & + & 1 & . & 1 & 1 & 1 & 3 & 1 & 2 & + & 1 \\
\hline Blechnum spicant & 1 & 2 & 1 & 1 & 1 & 1 & 1 & . & 2 & 2 & 1 & 2 & 1 & 3 & 1 & 1 & 2 & 2 & 2 & 1 & 2 & 1 & 3 & 1 \\
\hline Athyrium filix-femina & 3 & 1 & 1 & 1 & 3 & 1 & 4 & 3 & 2 & 3 & 3 & 2 & 3 & . & 2 & . & 3 & 2 & 1 & 3 & 4 & 2 & 1 & 2 \\
\hline Teucrium scorodonia & 1 & 1 & 1 & 2 & 2 & 1 & 1 & 2 & + & 1 & 1 & 2 & . & 3 & . & . & + & 1 & 1 & + & 2 & 1 & + & + \\
\hline Dryopteris affinis & 2 & + & 1 & + & 1 & . & + & 1 & + & 1 & 2 & 1 & . & 1 & 1 & 2 & 2 & 1 & + & 1 & 1 & . & + & 1 \\
\hline Euphorbia dulcis & 1 & 1 & 1 & 1 & . & 1 & + & 1 & 2 & 2 & 2 & 2 & . & . & + & 1 & . & 1 & 1 & 1 & 1 & 1 & 1 & 1 \\
\hline Lonicera hispanica & 1 & 1 & 1 & + & . & · & · & 1 & + & 1 & . & 1 & 2 & 1 & 1 & 1 & 1 & 1 & 1 & · & 1 & 1 & 1 & 1 \\
\hline Deschampsia subtriflora & . & . & 1 & 2 & 1 & 1 & + & + & + & . & 1 & 1 & 2 & $\mathrm{r}$ & 1 & . & 1 & 1 & 1 & + & . & 1 & + & . \\
\hline Holcus mollis & + & . & 1 & 1 & 1 & · & . & . & . & 1 & 1 & 1 & 1 & 3 & 1 & 2 & 1 & 1 & 1 & 1 & + & 2 & 1 & + \\
\hline Hedera hibernica & . & . & 2 & 1 & . & · & 1 & 1 & . & 1 & 2 & 1 & + & 1 & 1 & 1 & 1 & 2 & . & . & 1 & $\cdot$ & 1 & 1 \\
\hline Senecio nemorensis & 1 & . & . & 1 & . & 1 & 2 & 2 & . & . & 2 & 2 & . & + & 2 & 1 & . & 1 & 2 & . & 1 & 1 & . & 1 \\
\hline Oenanthe crocata & . & . & 2 & + & 1 & . & + & 1 & . & 1 & 3 & 3 & 1 & . & . & . & + & · & . & . & + & 1 & 1 & . \\
\hline Carex reuteriana & . & . & + & 1 & . & 1 & 1 & + & . & 1 & . & . & . & $\mathrm{r}$ & . & . & . & . & 2 & . & + & 1 & . & . \\
\hline Osmunda regalis & . & · & . & + & . & . & + & 1 & + & . & . & . & . & . & . & . & · & . & . & 1 & + & + & . & 2 \\
\hline Dryopteris aemula & . & · & . & . & . & . & . & . & + & . & . & . & . & . & . & . & 2 & 1 & . & . & . & . & . & + \\
\hline Ranunculus ficaria & . & + & . & . & . & . & . & . & . & . & . & . & . & . & . & + & . & . & . & . & . & . & . & + \\
\hline Carex remota & + & · & · & · & · & . & . & · & · & . & . & · & · & · & · & . & $\cdot$ & $\cdot$ & . & · & · & · & · & + \\
\hline Polystichum setiferum & + & . & . & . & . & . & . & . & . & . & . & . & . & . & . & · & . & . & . & . & . & . & . & . \\
\hline Myosotis martinii & . & $\cdot$ & $\cdot$ & $\cdot$ & . & . & $\cdot$ & . & . & . & . & . & · & . & · & + & . & . & . & . & · & · & $\cdot$ & $\cdot$ \\
\hline \multicolumn{25}{|c|}{ Characteristics of Quercetea robori-petraeae and Carpino-Fagetea } \\
\hline Viola riviniana & + & · & 1 & 1 & · & 1 & 1 & 1 & · & + & 1 & · & + & 1 & + & . & + & . & . & 1 & 1 & 1 & . & + \\
\hline Stellaria holostea & 2 & . & 1 & . & . & . & + & + & . & . & + & + & . & 1 & 1 & 1 & . & 1 & . & . & . & . & 1 & . \\
\hline Dryopteris dilatata & 1 & 2 & 1 & . & . & . & . & . & . & . & + & + & . & + & . & 2 & 1 & . & . & . & . & . & . & 1 \\
\hline Polypodium vulgare & . & . & . & + & . & . & . & . & . & + & . & . & . & . & + & + & + & + & . & . & + & . & + & + \\
\hline Ajuga reptans & 2 & . & . & 1 & . & . & 1 & . & . & . & . & + & . & . & . & 1 & · & 2 & . & . & . & . & . & + \\
\hline Crepis lampsanoides & . & . & . & + & . & + & 1 & 1 & . & + & 1 & + & . & . & . & . & · & $\cdot$ & . & . & . & . & . & $\cdot$ \\
\hline Avenella flexuosa s.l. & . & · & . & 1 & . & . & . & . & . & . & . & + & . & + & . & . & . & 1 & . & + & . & . & . & 2 \\
\hline Vaccinium myrtillus & $\cdot$ & $\cdot$ & · & $\cdot$ & · & · & . & . & 1 & . & . & . & · & · & 1 & + & 1 & 1 & . & . & $\cdot$ & $\cdot$ & · & 1 \\
\hline Saxifraga spathularis & 1 & · & . & 2 & . & . & . & . & . & · & . & . & · & . & . & 2 & . & 2 & . & . & · & · & · & + \\
\hline Oxalis acetosella & 1 & . & . & 1 & · & . & . & . & . & . & . & · & · & . & . & 1 & . & 2 & . & · & · & · & · & + \\
\hline Luzula sylvatica & . & · & . & 2 & . & . & 1 & 1 & . & . & . & . & . & . & . & . & · & . & . & . & · & · & . & + \\
\hline Euphorbia amygdaloides & . & . & . & . & 1 & . & 1 & 1 & . & . & . & . & . & . & . & . & . & . & . & · & 1 & . & . & · \\
\hline Lathyrus linifolius & $\cdot$ & . & . & 1 & . & . & . & . & . & . & . & . & . & . & + & . & . & . & . & + & . & . & . & . \\
\hline Melampyrum pratense & $\cdot$ & · & $\cdot$ & $\cdot$ & . & · & . & . & + & + & . & · & · & . & . & $\cdot$ & 1 & $\cdot$ & . & · & $\cdot$ & $\cdot$ & · & $\cdot$ \\
\hline $\begin{array}{l}\text { Anemone nemorosa } \\
\text { Other species }\end{array}$ & . & . & . & . & . & . & . & . & . & . & . & . & . & . & . & + & + & . & . & . & . & . & . & + \\
\hline Rubus sp. & 3 & 2 & 2 & . & 1 & 1 & 2 & 1 & + & 1 & 1 & 1 & 1 & 2 & 1 & 1 & 1 & 1 & + & + & 2 & 1 & 1 & 2 \\
\hline
\end{tabular}




\begin{tabular}{|c|c|c|c|c|c|c|c|c|c|c|c|c|c|c|c|c|c|c|c|c|c|c|c|c|}
\hline Brachypodium pinnatum & . & . & 3 & 1 & 3 & 2 & 3 & 3 & $\cdot$ & 3 & 3 & 4 & 3 & 1 & 1 & $\cdot$ & $\cdot$ & + & 4 & 2 & 4 & 2 & . & + \\
\hline Dactylis glomerata & . & + & . & 1 & 1 & . & $\cdot$ & + & $\cdot$ & 1 & + & + & 1 & + & 1 & + & . & + & . & . & $\cdot$ & 1 & . & + \\
\hline Pteridium aquilinum & . & . & . & $\mathrm{r}$ & . & . & + & . & 1 & 1 & $\cdot$ & 1 & + & 2 & $\cdot$ & $\cdot$ & + & + & + & 1 & 2 & + & . & \\
\hline Agrostis capillaris & . & 2 & 1 & 1 & . & . & $\cdot$ & 1 & 2 & $\cdot$ & 1 & 1 & 1 & $\cdot$ & 1 & · & . & 1 & 1 & 1 & $\cdot$ & 1 & 2 & \\
\hline Omphalodes nitida & + & . & . & . & . & + & + & 1 & . & 1 & 1 & 1 & $\cdot$ & + & $\cdot$ & $\cdot$ & + & 2 & + & . & 2 & $\cdot$ & & + \\
\hline Digitalis purpurea & . & 1 & + & $\mathrm{r}$ & + & $\cdot$ & + & + & . & $\mathrm{r}$ & + & + & $\cdot$ & + & $\cdot$ & . & + & + & + & . & $\cdot$ & + & & \\
\hline Centaurea rivularis & . & . & . & 1 & + & + & $\mathrm{r}$ & + & . & + & + & + & + & $\cdot$ & + & . & . & $\cdot$ & 1 & + & $\cdot$ & + & $\cdot$ & \\
\hline Angelica major & . & 1 & . & . & 1 & + & $\cdot$ & + & $\cdot$ & $\cdot$ & + & $\mathrm{r}$ & + & + & 1 & . & . & + & . & . & $\cdot$ & + & . & \\
\hline Ranunculus repens & 1 & . & . & + & 1 & $\cdot$ & $\cdot$ & 1 & $\cdot$ & $\cdot$ & + & $\cdot$ & + & $\cdot$ & 1 & . & . & + & $\cdot$ & . & $\cdot$ & + & $\cdot$ & + \\
\hline Crocus serotinus & . & . & . & + & . & $\cdot$ & + & $\cdot$ & . & $\cdot$ & $\cdot$ & + & $\cdot$ & + & + & $\cdot$ & $\cdot$ & $\cdot$ & . & . & + & + & + & \\
\hline Carex laevigata & + & . & . & 1 & . & + & $\cdot$ & $\cdot$ & $\cdot$ & $\cdot$ & $\cdot$ & $\cdot$ & $\cdot$ & $\cdot$ & $\cdot$ & + & + & + & $\cdot$ & . & $\cdot$ & $\cdot$ & + & + \\
\hline Cardamine pratensis & . & . & . & 1 & . & + & . & . & . & + & $\cdot$ & $\cdot$ & $\cdot$ & $\cdot$ & $\cdot$ & 1 & $\cdot$ & 1 & $\cdot$ & . & + & $\cdot$ & . & + \\
\hline Arrhenatherum bulbosum & . & . & . & . & 2 & . & + & $\cdot$ & $\cdot$ & $\cdot$ & 1 & $\cdot$ & $\cdot$ & $\cdot$ & 1 & $\cdot$ & $\cdot$ & . & 1 & . & 1 & 1 & $\cdot$ & \\
\hline Caltha palustris & . & . & . & . & . & $\cdot$ & + & $\cdot$ & . & 1 & $\cdot$ & + & $\cdot$ & $\cdot$ & + & + & $\cdot$ & $\cdot$ & . & . & $\cdot$ & 1 & . & \\
\hline Scrophularia auriculata & . & . & + & . & . & $\cdot$ & $\cdot$ & + & $\cdot$ & $\cdot$ & + & $\cdot$ & + & $\cdot$ & 1 & $\cdot$ & $\cdot$ & $\cdot$ & $\cdot$ & . & $\cdot$ & $\cdot$ & + & \\
\hline Lamium maculatum & . & . & 1 & . & . & . & . & + & $\cdot$ & $\cdot$ & + & $\cdot$ & $\cdot$ & $\cdot$ & 1 & . & $\cdot$ & + & $\cdot$ & . & $\cdot$ & $\cdot$ & . & \\
\hline Peucedanum lancifolium & . & . & . & . & . & $\cdot$ & $\cdot$ & $\cdot$ & + & $\cdot$ & $\cdot$ & $\cdot$ & 1 & $\cdot$ & + & . & $\cdot$ & $\cdot$ & $\cdot$ & . & $\cdot$ & + & . & + \\
\hline Prunella vulgaris & . & . & . & 1 & . & . & . & . & . & + & + & $\cdot$ & 1 & . & + & . & $\cdot$ & $\cdot$ & $\cdot$ & . & $\cdot$ & $\cdot$ & . & \\
\hline Galium palustre & . & . & . & · & + & . & + & + & . & $\cdot$ & $\cdot$ & $\cdot$ & 1 & $\cdot$ & $\cdot$ & $\cdot$ & $\cdot$ & $\cdot$ & $\cdot$ & + & $\cdot$ & $\cdot$ & $\cdot$ & \\
\hline Juncus effusus & + & . & . & . & . & + & + & $\cdot$ & . & $\cdot$ & $\cdot$ & $\cdot$ & $\cdot$ & $\cdot$ & $\cdot$ & + & $\cdot$ & $\cdot$ & $\cdot$ & . & $\cdot$ & $\cdot$ & $\cdot$ & + \\
\hline Anthoxanthum amarum & . & . & . & 2 & . & . & $\cdot$ & . & · & 1 & $\cdot$ & + & $\cdot$ & $\cdot$ & $\cdot$ & $\cdot$ & $\cdot$ & $\cdot$ & 1 & . & $\cdot$ & $\cdot$ & $\cdot$ & \\
\hline Potentilla erecta & . & . & . & . & . & + & . & . & + & + & $\cdot$ & $\cdot$ & $\cdot$ & $\cdot$ & $\cdot$ & . & $\cdot$ & $\cdot$ & $\cdot$ & . & $\cdot$ & + & + & \\
\hline Molinia caerulea & . & . & . & . & . & . & . & . & 1 & $\cdot$ & $\cdot$ & $\cdot$ & 1 & $\cdot$ & $\cdot$ & $\cdot$ & $\cdot$ & $\cdot$ & $\cdot$ & 1 & $\cdot$ & 1 & + & \\
\hline Cirsium filipendulum & . & . & . & . & . & . & . & + & $\cdot$ & $\cdot$ & $\cdot$ & $\cdot$ & $\cdot$ & $\cdot$ & 1 & . & $\cdot$ & . & . & + & $\cdot$ & + & . & \\
\hline Polygonum hydropiper & . & . & . & . & . & . & . & + & $\cdot$ & . & + & + & + & $\cdot$ & + & . & $\cdot$ & · & $\cdot$ & . & $\cdot$ & $\cdot$ & . & \\
\hline Carex pilulifera & . & . & . & . & . & . & . & $\cdot$ & $\cdot$ & . & $\cdot$ & $\cdot$ & $\cdot$ & + & $\cdot$ & . & $\cdot$ & . & . & + & $\cdot$ & $\cdot$ & + & + \\
\hline Epilobium obscurum & . & . & . & . & . & . & + & $\cdot$ & $\cdot$ & . & + & $\cdot$ & + & $\cdot$ & 1 & . & $\cdot$ & . & . & . & $\cdot$ & $\cdot$ & . & \\
\hline Rumex acetosa & + & . & . & 1 & . & . & $\cdot$ & . & $\cdot$ & . & $\cdot$ & $\cdot$ & $\cdot$ & $\cdot$ & + & . & $\cdot$ & . & . & . & $\cdot$ & $\cdot$ & . & \\
\hline Anthoxanthum odoratum & . & . & . & . & . & . & . & . & + & + & $\cdot$ & $\cdot$ & $\cdot$ & $\cdot$ & $\cdot$ & $\cdot$ & 1 & . & . & . & $\cdot$ & $\cdot$ & . & \\
\hline Lastrea limbosperma & . & . & . & . & . & . & . & . & . & . & $\cdot$ & + & $\cdot$ & $\cdot$ & $\cdot$ & 1 & $\cdot$ & . & . & . & $\cdot$ & $\cdot$ & . & 1 \\
\hline Lotus corniculatus & · & . & . & . & . & · & $\cdot$ & $\cdot$ & $\cdot$ & $\cdot$ & + & + & $\cdot$ & $\cdot$ & 1 & $\cdot$ & $\cdot$ & $\cdot$ & $\cdot$ & · & $\cdot$ & $\cdot$ & $\cdot$ & \\
\hline Lotus pedunculatus & + & + & . & . & . & . & . & . & $\cdot$ & $\cdot$ & $\cdot$ & $\cdot$ & $\cdot$ & $\cdot$ & $\cdot$ & . & . & . & . & . & . & $\cdot$ & . & + \\
\hline Heracleum sphondylium & . & . & . & + & . & + & $\cdot$ & $\cdot$ & $\cdot$ & $\cdot$ & $\cdot$ & $\cdot$ & $\cdot$ & $\cdot$ & $\cdot$ & . & $\cdot$ & $\cdot$ & $\cdot$ & . & $\cdot$ & $\cdot$ & $\cdot$ & \\
\hline Rumex obtusifolius & . & . & . & . & . & . & + & + & $\cdot$ & $\cdot$ & $\cdot$ & $\cdot$ & $\cdot$ & $\cdot$ & + & $\cdot$ & . & . & . & . & $\cdot$ & $\cdot$ & . & \\
\hline Cardamine hirsuta & . & . & . & . & . & . & . & + & $\cdot$ & $\cdot$ & $\cdot$ & + & . & . & $\cdot$ & + & $\cdot$ & $\cdot$ & . & . & . & $\cdot$ & . & \\
\hline
\end{tabular}

Other species: Characteristics of Quercetea robori-petraeae and Carpino-Fagetea: Pulmonaria longifolia + in 16 and $18 ;$ Primula acaulis + in $1 ;$ Stachys officinalis 1 in 4; Ranunculus tuberosus 1 in 5; Dryopteris filix-mas 1 in 6; Hieracium murorum + in 20; Hypericum androsaemum + in 24. Other species: Silene dioica + in 1 and 16; Ulex gallii + in 2 and 23; Wahlenbergia hederacea + in 2 and 15; Valeriana dioica 1 in 5 and 1 in 10; Scrophularia herminii + in 7 and 8; Geranium robertianum + in 8 and 24; Narcissus asturiensis + and Festuca gr. rubra 1 in 9 and 10; Sphagnum sp. 2 in 9 and 1 in 24; Urtica dioica + in 11 and 12; Sibthorpia europaea + in 13 and 15; Cirsium palustre $\mathrm{r}$ in 16 and 18; Polytrichum commune + in 16 and 24; Avenula sulcata 1 in 17 and 2 in 23; Silene latifolia + in 3; Adenostyles alpina 1, Allium victorialis and Prunella grandiflora + in 4 ; Saxifraga lepismigena 1 in 6; Holcus lanatus and Solanum nigrum + in 8; Carex cariophyllea and Carex echinata 1, Danthonia decumbens, Erica mackayana and Narthecium ossifragum + in 9; Cytisus commutatus, Hyacinthoides non-scripta, Physospermum cornubiense, Pseudarrhenatherum longifolium and Ulex minor + in 10; Plantago major $\mathrm{r}$ in 12; Scutellaria minor 1, Lapsana communis, Stellaria sp. and Succisa pratensis + in 13; Agrostis stolonifera + in 14; Galium papillosum and Trifolium repens + in 15; Chaerophyllum hirsutum 2, Mnium undulatum 1 and Epilobium sp. + in 16; Veronica chamaedrys + in 18; Valeriana montana + in 19; Calluna vulgaris + in 20; Carex sp., Lythrum salicaria and Quercus rubra (sd1.) + in 21; Juncus bulbosus + in 22; Chrysosplenium oppositifolium and Pellia sp. + in 24.

Localities (geographical coordinates: UTM, datum ETRS 89, 29T, in brackets): 1: A Coruña: Sobrado dos Monxes, Grixalba, Río Lombao, downstream from Ponte Tuela (582, 4771); 2: Lugo: A Fonsagrada, Carballido, between Vilar de Calvos and A Burela, Rego de Chaves (649, 4788); 3: A Coruña: Aranga, Cambás, between Boade and A Lousa, Regato da Lousa (586, 4790); 4: A Coruña: Aranga, A Vide, Rego da Loba, downstream from Ponte Chanca (584, 4790); 5: Lugo: Muras, between A Ínsua and Arealba, Río Eume (609, 4811); 6: Lugo: Guitiriz, Rego de Requeixo, downstream from Buriz (593, 4789); 7: Lugo: Vilalba, San Simón da Costa, A Fontarraña, Rego de Laxoso (610, 4804); 8: Lugo: Xermade, Río Labrada, between Mollafariña and Lugar Vello, upstream from Ponte Corval (597, 4795); 9: Lugo: Vilalba, between Cazás and Buriz, Río Labrada (597, 4796); 10: A Coruña: Irixoa, Verís, O Requeixo, lower limit of Fraga do Monte do Raño, Río Zarzo (581, 4792); 11: A Coruña: Aranga, A Reborica, Rego da Costa (582, 4783); 12: Lugo: Xermade, Rego de Porto do Souto, downstream from O Souto (593, 4806); 13: Lugo: Xermade, Río de Porto Liñares, downstream from A Poupariña (593, 4798); 14: Lugo: Guitiriz, Arxá, Río Escádebas (590, 4785); 15: Lugo: Xermade, Río de Tordegos, in front of Os Miraces (594, 4803); 16: Lugo: Friol, A Roxica, Rego da Cabana (589, 4768); 17: Lugo: A Pontenova, Bogo, rivulet between O Barreiro and Río de Campos (651, 4792); 18: A Coruna: Irixoa, Verís, Río Zarzo, upstream from O Requeixo (580, 4792); 19: Lugo: Muras, Bustelo, Rego das Ferreiras (602, 4815 20: Lugo: Guitiriz, Río Labrada, downstream from O Pumariño (588, 4794); 21: Lugo: Guitiriz, Santo Estevo de Parga, Rego da Roxida, downstream from Muíño de Fraguas (591, 4776); 22: Lugo: Vilalba, Belesar, Río Labrada, between A Penadanta and O Foro $(600,4793)$; 23: Lugo: Xermade, Río Labrada, downstream from the bridge between Parga and Momán (592, 4795); 24: Lugo: Palas de Rei, A Cernada, Rego da Corda (594, 4758); 25: Lugo: Meira, Seixosmil, Carballal das Carrieiras, Rego da Pena Vella $(642,4785)$. 
Table 3 (cont.). Violo palustris-Betuletum pubescentis Rodríguez Guitián 2010

1-5: typical variant, Salix atrocinerea facies; 6-13: Alnus glutinosa variant, typical facies;

14: Alnus glutinosa variant, Salix atrocinerea facies.

(Alno glutinosae-Populetea albae, Alno-Fraxinetalia excelsioris, Hyperico-Alnion glutinosae)

\begin{tabular}{|c|c|c|c|c|c|c|c|c|c|c|c|c|c|c|}
\hline Altitude (m asl) & 770 & 450 & 770 & 620 & 605 & 445 & 425 & 580 & 530 & 420 & 400 & 455 & 485 & 490 \\
\hline Slope $\left({ }^{\circ}\right)$ & 6 & $<2$ & 4 & 4 & 8 & $<2$ & 2 & 6 & 4 & $<2$ & $<2$ & $<2$ & $<2$ & $<2$ \\
\hline Exposition & E & $\mathrm{NE}$ & $\mathrm{N}$ & SSW & ESE & SE & NW & SW & $\mathrm{NE}$ & $\mathrm{SE}$ & SSE & SE & $\mathrm{NE}$ & SE \\
\hline Height (m) & $8-10$ & 16 & $6-10$ & $8-12$ & 8 & 15 & 14 & 16 & 22 & 20 & 20 & 16 & 14 & 10 \\
\hline Cover $\mathrm{E}_{1}(\%)$ & 100 & 100 & 100 & 100 & 90 & 90 & 100 & 95 & 100 & 100 & 85 & 90 & 100 & 100 \\
\hline Cover $\mathrm{E}_{2}(\%)$ & 20 & 35 & 15 & 15 & 35 & 15 & 25 & 25 & $<5$ & 25 & 20 & 10 & 25 & 10 \\
\hline Cover $\mathrm{E}_{3}^{2}(\%)$ & 95 & 100 & 95 & 90 & 70 & 100 & 90 & 85 & 95 & 100 & 95 & 100 & 100 & 90 \\
\hline Area $\left(\mathrm{m}^{2}\right)$ & 300 & 300 & 300 & 300 & 200 & 400 & 450 & 300 & 500 & 100 & 500 & 300 & 160 & 300 \\
\hline N. species & 45 & 44 & 50 & 36 & 42 & 42 & 44 & 35 & 54 & 25 & 58 & 28 & 33 & 33 \\
\hline N. relevé & 1 & 2 & 3 & 4 & 5 & 6 & 7 & 8 & 9 & 10 & 11 & 12 & 13 & 14 \\
\hline \multicolumn{15}{|l|}{$\mathrm{E}_{1}(>4,0 \mathrm{~m})+\mathrm{E}_{2}(1,5-4,0 \mathrm{~m})$} \\
\hline Betula pubescens & 2 & 2 & 1 & 2 & . & 5 & 5 & 3 & 4 & 4 & 4 & 4 & 3 & 2 \\
\hline Quercus robur & 1 & 2 & . & 1 & . & + & 1 & 3 & 2 & 1 & 1 & 1 & 1 & . \\
\hline Erica arborea & + & 1 & 1 & . & 1 & + & 1 & 1 & + & 1 & . & . & 1 & . \\
\hline Frangula alnus & + & . & . & . & . & + & + & 1 & + & 1 & . & 1 & 1 & + \\
\hline Cytisus scoparius & . & . & . & + & . & + & 1 & + & + & . & . & . & . & . \\
\hline Pyrus cordata & 1 & . & . & . & + & . & . & . & . & . & . & . & + & . \\
\hline Ilex aquifolium & + & . & . & . & . & . & + & 1 & . & . & . & . & . & . \\
\hline Sambucus nigra & . & . & . & . & . & . & 1 & . & . & . & + & . & 1 & . \\
\hline Corylus avellana & 1 & . & 2 & . & . & . & . & . & . & . & . & . & . & . \\
\hline Acer pseudoplatanus & + & 1 & . & . & . & . & . & . & . & . & . & . & . & . \\
\hline Fraxinus excelsior & . & $\cdot$ & 1 & . & . & . & $\cdot$ & . & $\cdot$ & . & $\mathrm{r}$ & . & . & . \\
\hline Castanea sativa & . & 1 & . & . & . & . & . & . & . & . & . & . & . & . \\
\hline Genista florida & . & $\cdot$ & . & . & . & . & + & . & . & . & . & . & . & $\cdot$ \\
\hline Sorbus aucuparia & + & . & . & . & . & . & . & . & . & . & . & . & . & $\cdot$ \\
\hline Crataegus monogyna & . & 1 & . & . & . & . & . & . & . & . & . & . & . & . \\
\hline Laurus nobilis & . & . & . & . & . & . & + & . & . & . & . & . & . & . \\
\hline Salix caprea & . & . & 1 & . & . & . & . & . & . & . & . & . & . & . \\
\hline \multicolumn{15}{|l|}{ Differentials of variant } \\
\hline Alnus glutinosa & . & . & . & . & . & + & 1 & 1 & 2 & 2 & 2 & 2 & 2 & 2 \\
\hline \multicolumn{15}{|l|}{ Differentials of facies } \\
\hline Salix atrocinerea & 4 & 4 & 4 & 5 & 5 & 2 & 2 & 3 & . & 1 & 2 & 2 & 2 & 5 \\
\hline \multicolumn{15}{|c|}{ Characteristcs of association and upper units $\mathrm{E}_{3}(<1,5 \mathrm{~m})$} \\
\hline Viola palustris & $1^{3}$ & + & + & + & 1 & + & 2 & 2 & 1 & 1 & 2 & 1 & 1 & 3 \\
\hline Athyrium filix-femina & 2 & . & 2 & 2 & 2 & 1 & 2 & 1 & 2 & 2 & 2 & . & 2 & 3 \\
\hline Blechnum spicant & 1 & 2 & 1 & 1 & 1 & + & 1 & 2 & 1 & 1 & . & . & 1 & 1 \\
\hline Dryopteris affinis & 1 & 1 & . & 3 & 1 & . & 1 & 1 & 1 & + & + & . & 1 & + \\
\hline Hedera hibernica & 1 & 2 & 1 & . & . & 1 & 1 & 1 & + & 1 & 2 & . & 2 & 2 \\
\hline Teucrium scorodonia & 1 & 1 & . & + & 1 & 2 & 1 & + & 1 & 1 & . & 2 & 1 & . \\
\hline Brachypodium rupestre & . & 4 & . & . & . & 5 & 1 & + & 3 & 4 & 4 & 5 & 4 & 1 \\
\hline Holcus mollis & 2 & 1 & . & 2 & 2 & 1 & 2 & . & 1 & . & 2 & 1 & . & . \\
\hline Euphorbia dulcis & 1 & . & 1 & 2 & . & + & 2 & 1 & 2 & . & 1 & . & 1 & + \\
\hline Lonicera hispanica & 1 & $\cdot$ & + & . & + & + & 2 & 1 & + & . & 1 & . & 2 & 2 \\
\hline Deschampsia subtriflora & . & 1 & + & 1 & . & + & 1 & 1 & 1 & + & 2 & . & . & 2 \\
\hline Senecio nemorensis & 1 & + & 1 & 2 & + & . & . & . & 2 & . & 1 & . & 1 & 2 \\
\hline Ranunculus ficaria & 1 & 2 & 1 & + & . & . & . & . & . & . & 1 & . & . & 1 \\
\hline Oenanthe crocata & . & 1 & . & . & . & + & . & . & 2 & + & 1 & . & 1 & 1 \\
\hline Carex reuteriana & . & . & . & . & . & 1 & . & 1 & 2 & . & . & 1 & . & . \\
\hline Myosotis martinii & . & . & + & 1 & + & + & $\cdot$ & $\cdot$ & $\cdot$ & . & 1 & $\cdot$ & . & $\cdot$ \\
\hline Dryopteris aemula & . & $\cdot$ & . & . & . & . & + & 1 & $\cdot$ & . & . & . & . & $\cdot$ \\
\hline Osmunda regalis & . & . & . & . & . & + & . & + & . & . & . & + & . & . \\
\hline \multicolumn{15}{|c|}{ Characteristics of Quercetea robori-petraeae and Carpino-Fagetea } \\
\hline Stellaria holostea & 1 & 1 & + & 2 & 1 & 1 & 1 & 1 & 1 & . & . & . & . & $\cdot$ \\
\hline Dryopteris dilatata & 2 & + & 1 & 1 & + & . & 1 & . & . & . & . & . & . & + \\
\hline Polypodium vulgare & . & + & . & · & 1 & . & · & + & + & . & + & . & + & + \\
\hline Viola riviniana & . & + & + & . & . & . & . & . & 1 & + & 1 & . & . & $\cdot$ \\
\hline Crepis lampsanoides & . & . & . & . & . & . & + & . & 1 & . & . & . & . & + \\
\hline Ajuga reptans & 2 & . & 1 & . & . & . & . & . & . & . & 1 & . & . & . \\
\hline Vaccinium myrtillus & 1 & . & + & . & . & . & . & 3 & . & . & . & . & . & $\cdot$ \\
\hline Avenella flexuosa s.l. & + & . & . & + & . & . & $\cdot$ & . & $\cdot$ & . & . & . & 1 & $\cdot$ \\
\hline Oxalis acetosella & 1 & . & 2 & . & 1 & . & . & . & . & . & . & . & . & . \\
\hline Hyacinthoides non-scripta & . & . & . & + & . & . & . & + & 2 & . & . & . & . & $\cdot$ \\
\hline \multicolumn{15}{|l|}{ Other species } \\
\hline Rubus sp. & 2 & 2 & + & 2 & 1 & 1 & 1 & 1 & 1 & 2 & + & 1 & 2 & 1 \\
\hline Pteridium aquilinum & $\mathrm{r}$ & 1 & + & . & . & 3 & 1 & + & $\mathrm{r}$ & 3 & 3 & 3 & 1 & . \\
\hline Dactylis glomerata & · & 1 & 1 & 1 & $\cdot$ & 2 & 1 & . & 1 & . & 1 & 2 & 1 & 1 \\
\hline Ranunculus repens & . & 1 & 1 & 1 & 1 & 1 & + & . & + & . & 1 & 1 & . & . \\
\hline Carex laevigata & + & + & + & + & + & . & . & . & + & . & + & . & + & + \\
\hline Angelica major & + & + & . & . & . & 1 & 1 & . & + & + & + & + & . & . \\
\hline
\end{tabular}




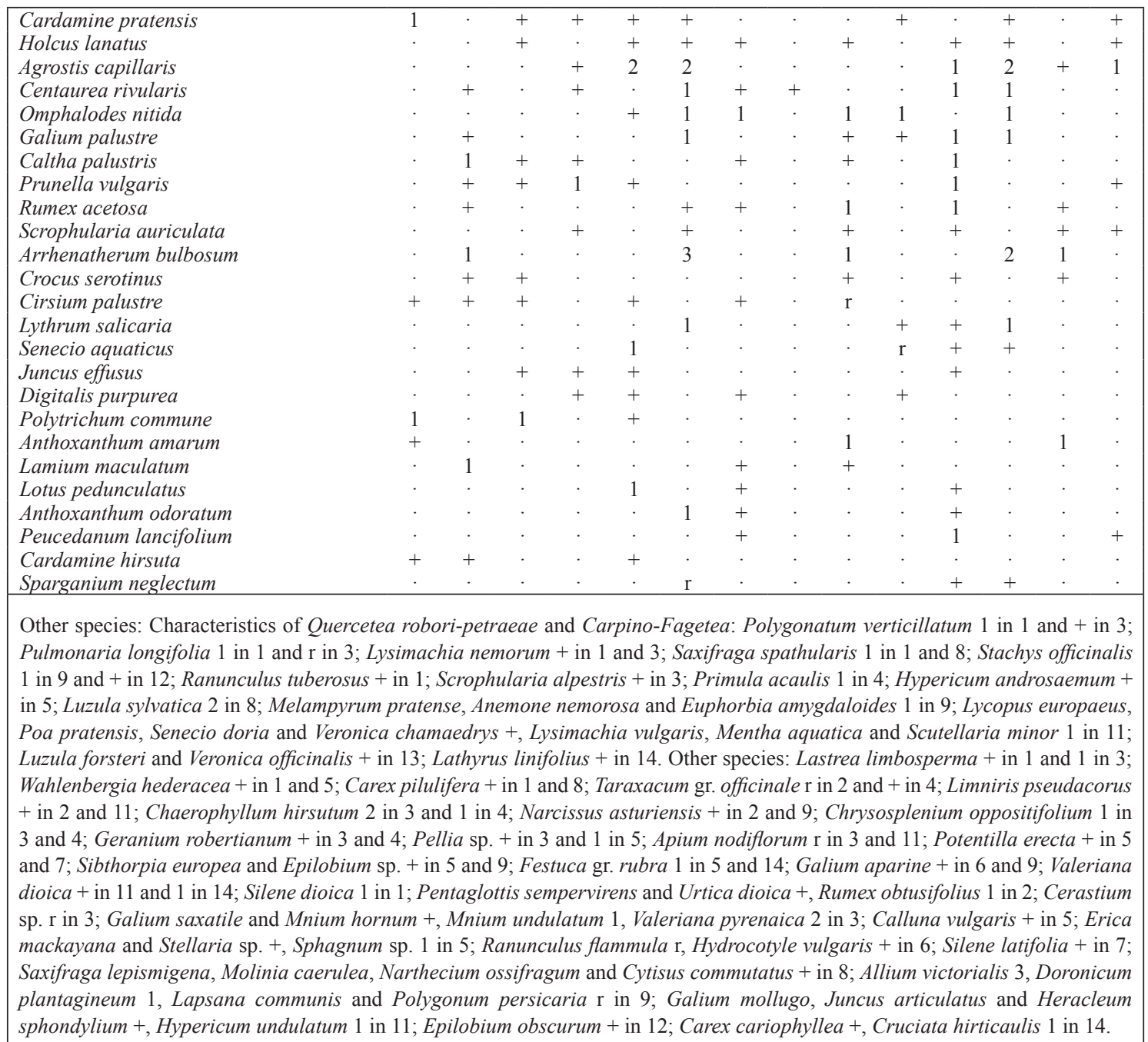

Localities (geographical coordinates: UTM, datum ETRS 89, 29T, in brackets): 1: Lugo: A Fonsagrada, Carballido, upper limit of Fraga de Reigadas, Rego do Mazo, (650, 4790); 2: Lugo: Xermade, between A Ribeira and Xermade, Rego de Vilariño (597, 4800); 3: Asturias: Taramundi, Turía, Rego das Pasadas (655, 4796); 4: Lugo: A Pastoriza, A Lagoa, Rego de Marful (628, 4799); 5: Lugo: A Pastoriza, Cadavedo, between Cadavedo and Alto do Fiouco, Rego do Batán (631, 4804); 6: Lugo: Gutiriz, Parga, between A Ponte and Rego de Revixoso, Río de Requeixo (594, 4786); 7: Coruña: Irixoa, Verís, Balcones, Río Zarzo (579, 4791); 8: Coruña: Aranga, Cambás, A Fraga do Castro, Rego de Portosalmallos (584, 4787); 9: Lugo: Friol, Roimil, between Nodar and Campo da Feira, River Parga. (593, 4768); 10: Lugo: Vilalba, Belesar, between A Chousa and O Larizo, Río de Silvela (601, 4791); 11: Lugo: Vilalba, Ponte Trimaz, River Trimaz (604, 4795); 12: Lugo: Guitiriz, Sal Salvador de Parga, Rego de Requeixo, downstream from Ponte de Báscuas (594, 4786); 13: Lugo: Friol, San Paio de Narla, River Narla(596, 4762); 14: Coruña: Oza-Cesuras, Rodeiro, Martín, Rego Martín, upstream from Braña da Zanca (575, 4779).

\section{Galician-Duriensian supratemperate ripari- an birch forests (Valeriano dioicae-Betuletum pubescentis ass. nova hoc loco)}

Riparian forests growing in the supratemperate horizon of the mountains in the GalicianDuriensian sector (Figure 1), on granitic and acidic metamorphic rocks (gneiss, slates and quartzites). Sorbus aucuparia and Salix atrocinerea are usually present, and Quercus pyrenaica and Frangula alnus are frequent.
In the understory Aquilegia dichroa, Angelica major, Athyrium filix-femina, Blechnum spicant, Cirsium palustre, Crepis lampsanoides, Erica arborea, Luzula sylvatica, Poanemoralis, Rubus sp., Stellaria holostea, Vaccinium mytillus, Viola riviniana and Valeriana officinalis are abundant. This community constitutes a new phytosociological association, named Valeriano dioicae-Betuletum pubescentis ass. nova hoc loco for which we select the relevé 18 in Table 5 as holotypus (Figure 5, photos 4a and 
4b). In view of their floristic composition, we consider that relevés of riparian birch forests taken by Gonçalves Aguiar (2000) in NE Portugal, Rodríguez Guitián \& Bariego Hernández (2009) in Sanabria Mountains and González de Paz (2013) in the Cabrera Range (Table 1, group 5b) match with this new phytosociological unit (Table 1, group 5a).

The floristic variations of this association (Table 5) are quite similar to those of association Chaerophyllo-Betuletum: there are typical,Alnus glutinosa and Sorbus aucuparia variants also with typical facies and, in the first and third variants, Salix atrocinerea facies, usually caused by human intervention.

Distribution: Galician-Duriensian sector.

Differential taxa with respect to other riparian birch forests: Pimpinella major, Rosa corymbifera, Stachys sylvatica, Streptopus amplexifolius, Viburnum opulus.

Differential taxa with respect to CariciBetuletum: Aconitum neapolitanum, Adenostyles alpina,Aquilegia vulgaris, Cardamine gallaecica, Ceratocapnos claviculata, Chaerophyllum hirsutum, Doronicum pubescens, Epilobium angustifolium, E.montanum, Helleborusfoetidus, Hypericumandrosaemum,Lysimachianemorum, Melampyrum pratense, Melica uniflora, Narcissus pseudonarcissus, Paris quadrifolia, Poa chaixii, $P$. nemoralis, Polygonatum verticillatum, Polystichum aculeatum, P. setiferum, Prunus padus, Pyrola minor, Rosa villosa, Rubus idaeus, Salix caprea, Sorbus aria, Thalictrum minus, Veratrum album.

Differential taxa with respect to Violo-Betuletum: Aconitum neapolitanum, Allium scorzonerifolium, Aquilegia dichroa, Calamagrostis arundinacea, Cardamine gallaecica, Doronicum pubescens, Epilobium angustifolium, E. montanum, Euphorbia hyberna, Festuca merinoi, Galium broterianum, Helleborus foetidus, Knautia nevadensis, Lilium martagon, Melica uniflora, Melittis melissophyllum, Myosotis cespitosa, Narcissus pseudonarcissus, Paradisea lusitanica, Paris quadrifolia, Poa chaixii, P. nemoralis, Polygonatum verticillatum, Polystichum aculeatum, P. setiferum, Potentilla sterilis, Prunus padus, Pyrola minor, Ranunculus platanifolius, Rosa villosa, Rubus idaeus, Salix caprea, Sorbus aria, Thalictrum minus, Th. speciosissimum, Valeriana officinalis, Veratrum album.

Differential taxa with respect to ChaerophylloBetuletum: Allium scorzonerifolium, Aquilegia dichroa, Calamagrostis arundinacea, Cirsium filipendulum, Galium broterianum, G. palustre, Knautianevadensis, Myosotiscespitosa,Oenanthe crocata, Paradisea lusitanica, Pyrus cordata,
Quercus robur, Scutellaria minor, Thalictrum speciosissimum, Valeriana officinalis.

\section{Syntaxonomic setting}

Biurrun et al. (2016) recently published a review of the syntaxonomy of the Iberian riparian forests and proposed a new hierarchical system of units for the Eurosiberian zone of Spain and N Portugal. In this work, information about NW Iberian birch forests published by Izco et al. (1986, LuzuloBetuletum subass. salicetosum atrocinereae), Gonçalves Aguiar (2000, "Betula celtibericaSalix atrocinerea community"), Honrado et al. (2003; Carici reuterianae-Betuletum celtibericae) and Rodríguez Guitián (2010) (Violo palustrisBetuletum pubescentis) as well as relevés of another riparian birch community (Rubo corylifoliiSalicetum atrocinereae subass. vaccinietosum myrtilli) described by Navarro (1989) from the Moncayo Massif (Iberian Range) were included in the data base. As a conclusion of the multivariate classification methods employed, these authors maintain the independence of Violo-Betuletum but considered the relevés of Izco et al. (1986), Gonçalves Aguiar (2002) and Navarro (1989) as belonging to Carici-Betuletum.

The new phytosociological information provided in this study contributes to clarify the floristic and syntaxonomic relations between these communities. We summarized it in a synthetic table (Table 1) from which the following conclusions stem:

- The NW Iberian relevés are clearly different from those collected by Navarro (1989) in the Iberian System. Many plants common in the former (some of them nothwestern iberian endemisms) were absent in the latter: Anemone trifolia subsp. albida, Aquilegia dichroa, Echium lusitanicum, Omphalodes nitida, Saxifraga spathularis, etc. and, conversely, several species present in the Moncayo forests are absent (Lysimachia vulgaris, Ranunculus acris, Sambucus racemosa, Sorbus torminalis) or with a very scarce presence (Carex divulsa, Erica vagans) in the studied NW Iberian riparian forests. To a great part, this could be a consequence of the distance between these two areas (almost $440 \mathrm{~km}$ in a straight line, see Figure 1). Consequently, we consider that the inclusion of both NW Iberian and Iberian Range forests in a single association, as proposed by Biurrun et al. (2016), is unjustified from both floristic and biogeographic points of view. 


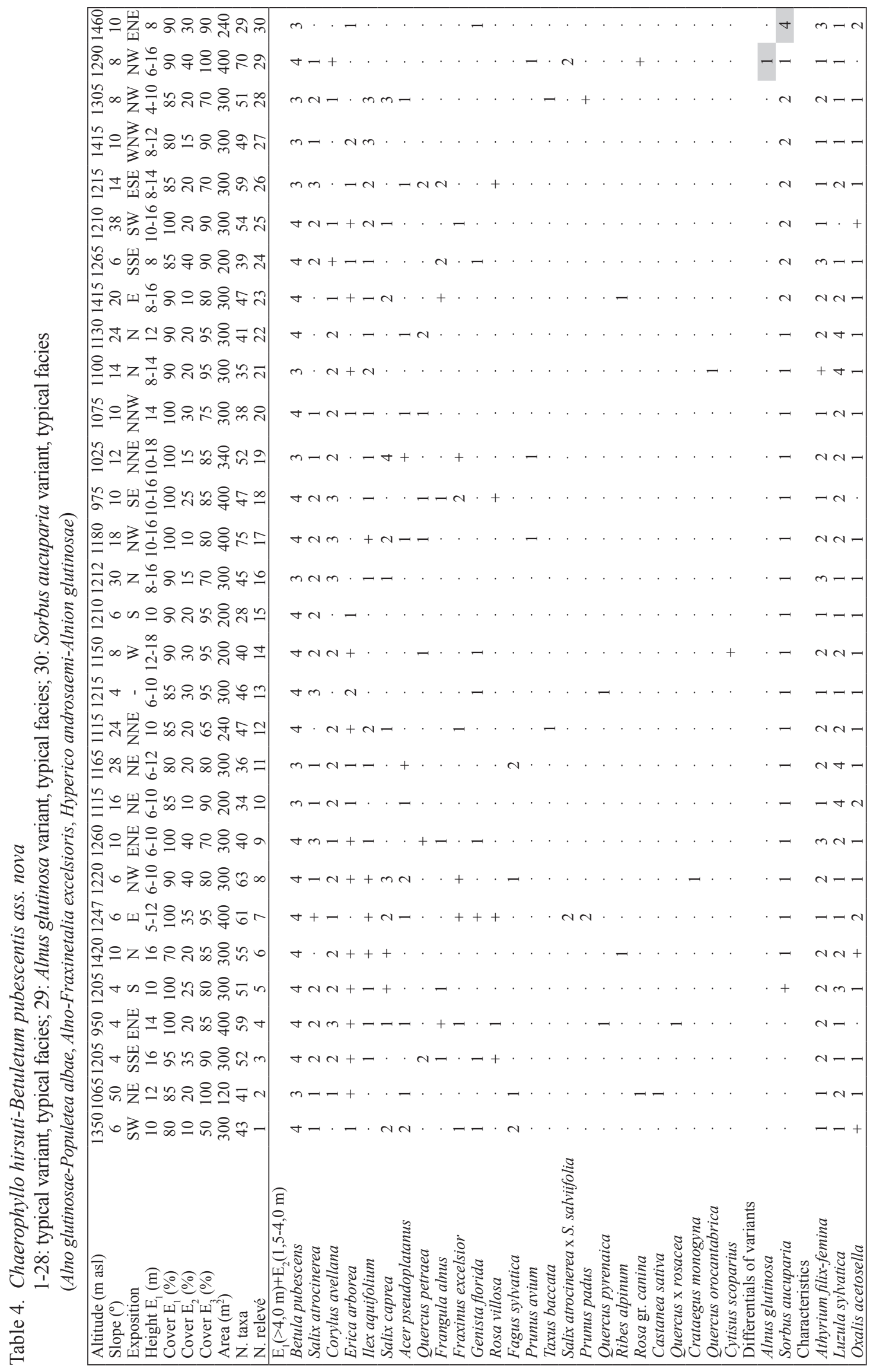




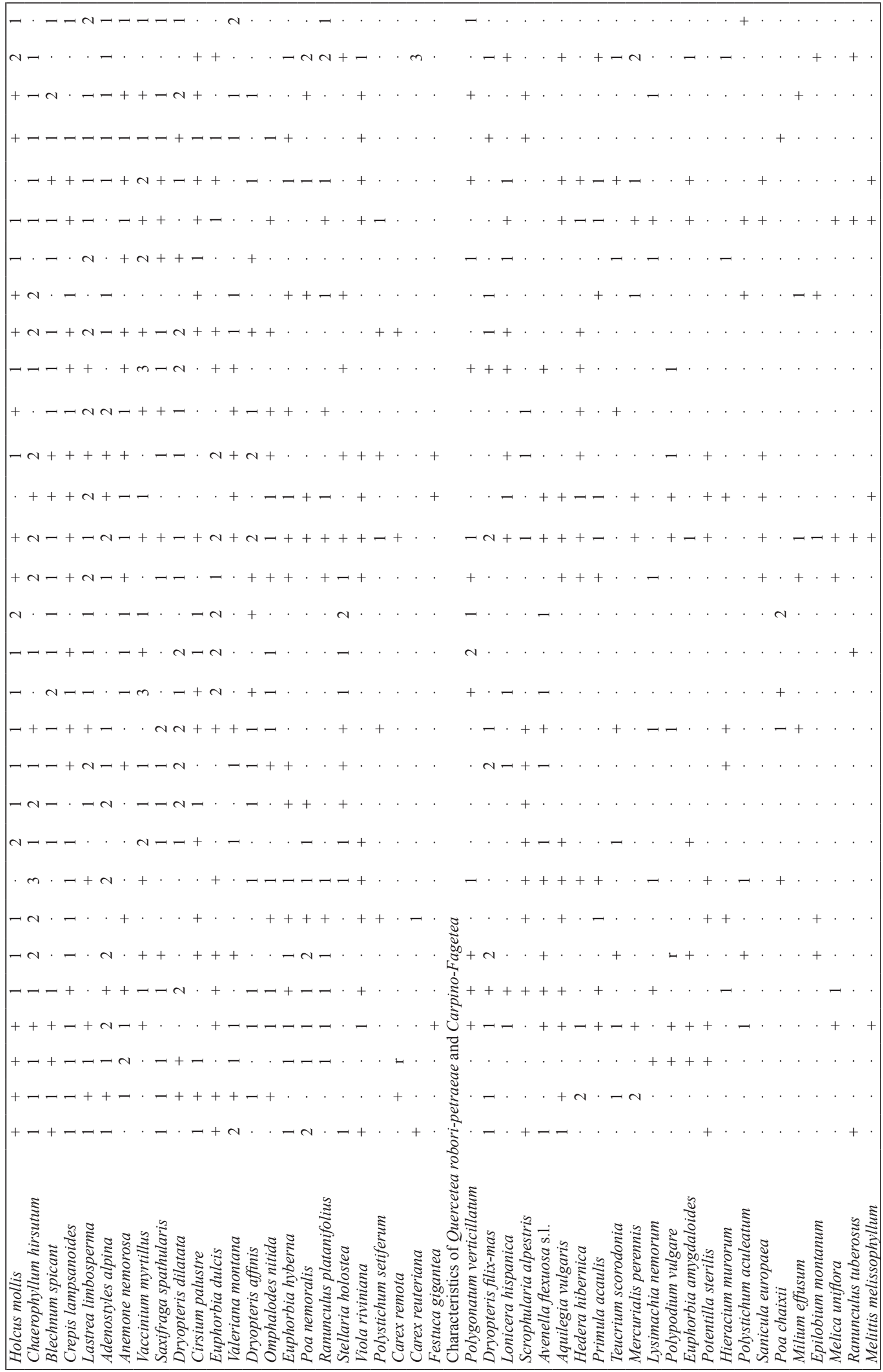




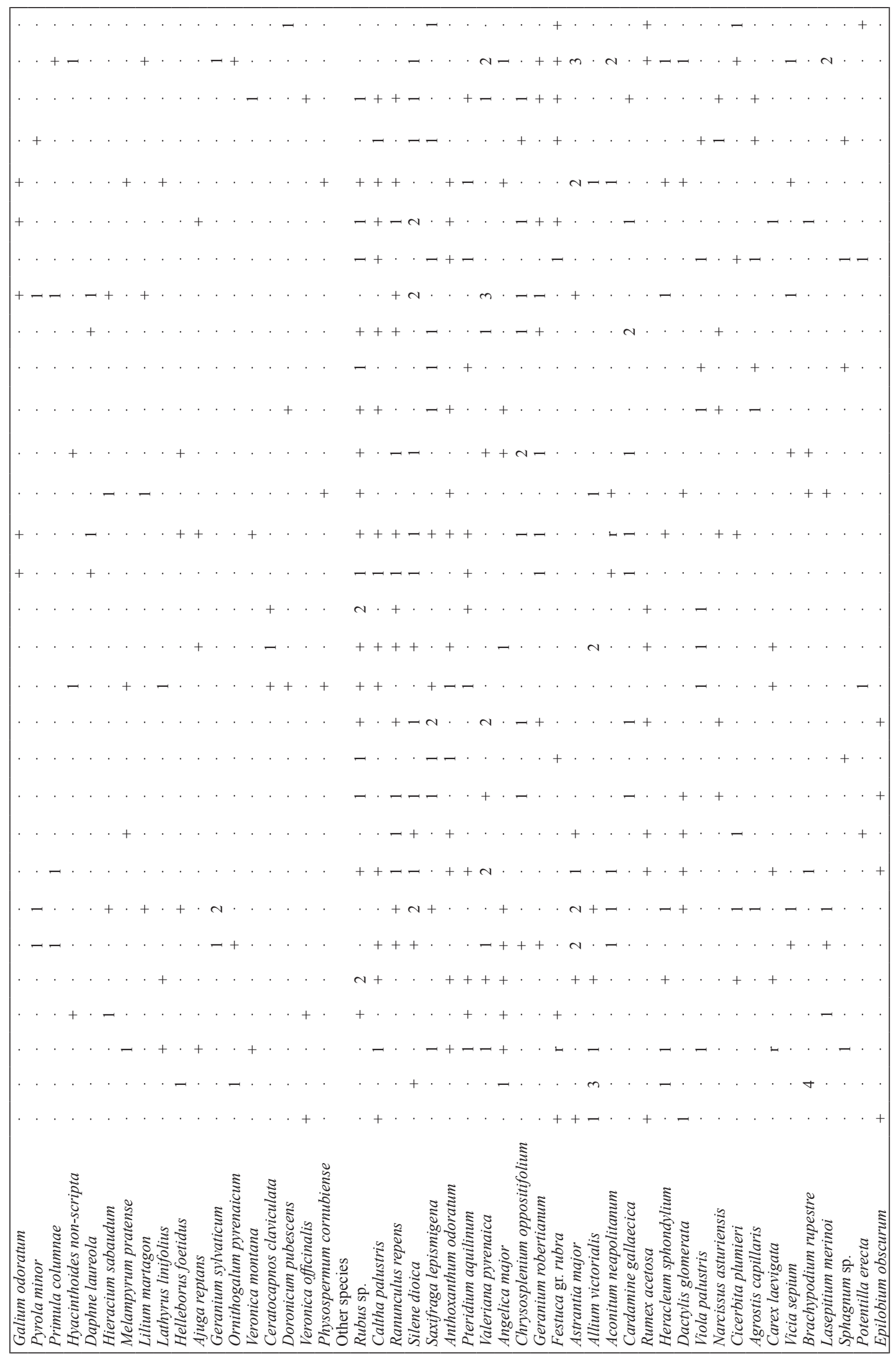




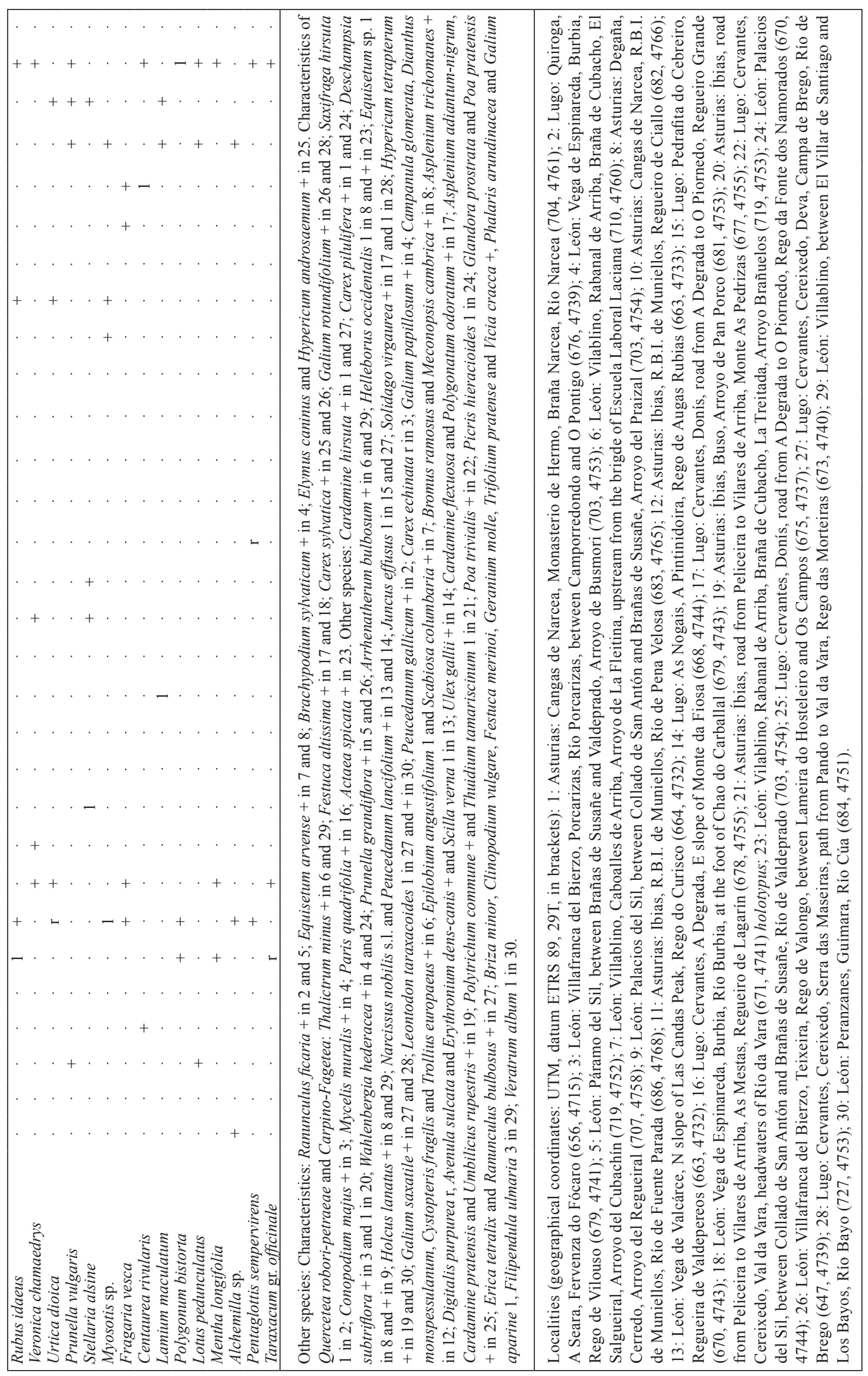


- In relation with the two relevés of the "Betula celtiberica and Salix atrocinerea community" described by Gonçalves Aguiar (2000) in the extreme NE Portugal, we believe that they must be included in the association Valeriano officinalis-Betuletum pubescentis because this is the only NW Iberian association with the simultaneous presence of Festuca merinoi, Poa nemoralis and Galium broterianum. Therefore, we consider the proposal of Biurrun et al. (2016, Appendix S3) of including them in Carici reuterianae-Betuletum celtibericae inappropriate.

- The same reasoning can be applied to the relevés collected in riparian birch forests of the Cabrera Range (León) and included into the LuzuloBetuletum subass. salicetosum atrocinereae by González de Paz (2012, Table 6.117). These relevés contain species that only appear in the association Valeriano officinalis-Betuletum (Rosa corymbifera, Streptopus amplexifolius or Stachys sylvatica) as well as others that this association shares with the Chaerophyllo hirsuti-Betuletum (Poa nemoralis, Epilobium montanum, Pyrola minor and Helleborus foetidus) but are always absent in the Carici reuterianae-Betuletum.

In relation to the syntaxonomic framework, Biurrun et al. (2016) proposed to gather the Iberian forests dominated by birches or common ash (Fraxinus excelsior) in the suballiance Saxifrago spathularis-Fraxinenion excelsioris, with Adenostyles alpina (sub A. alliariae subsp. hybrida), Betula pubescens (sub B. celtiberica), Blechnum spicant, Carex laevigata, Dryopteris dilatata, Holcus mollis, Luzula sylvatica (sub L. sylvatica subsp. henriquesii), Saxifraga spathularis, Vaccinium myrtillus and Viola palustris as differential taxa. As these taxa are more or less common in the four associations discussed above (Table 5), we agree with their proposal.

Following Biurrun et al. (2016), this suballiance should be included in the alliance Hyperico androsaemi-Alnion glutinosae, which has Culcita macrocarpa, Dryopteris aemula*, D. borreri, Hymenophyllum tunbrigense, Hypericum androsaemum*, Myosotis martini*, Phyllitis scolopendrium, Polystichum setiferum*, Saxifraga hirsuta, Scrophularia auriculata*, Senecio nemorensis* (sub. S. bayonnensis), Stegnogramma pozoi and Woodwardia radicans as differential taxa $(*$ identifies the taxa present in the studied forests).

Finally, these authors also proposed to include this alliance in the order AlnoFraxinetalia excelsioris Passarge 1968, belonging to the class Salici purpureaePopuletea nigrae (Rivas-Martínez \& Cantó ex Rivas-Martínez et al. 1991) Rivas-Martínez \& Cantó 2002. 


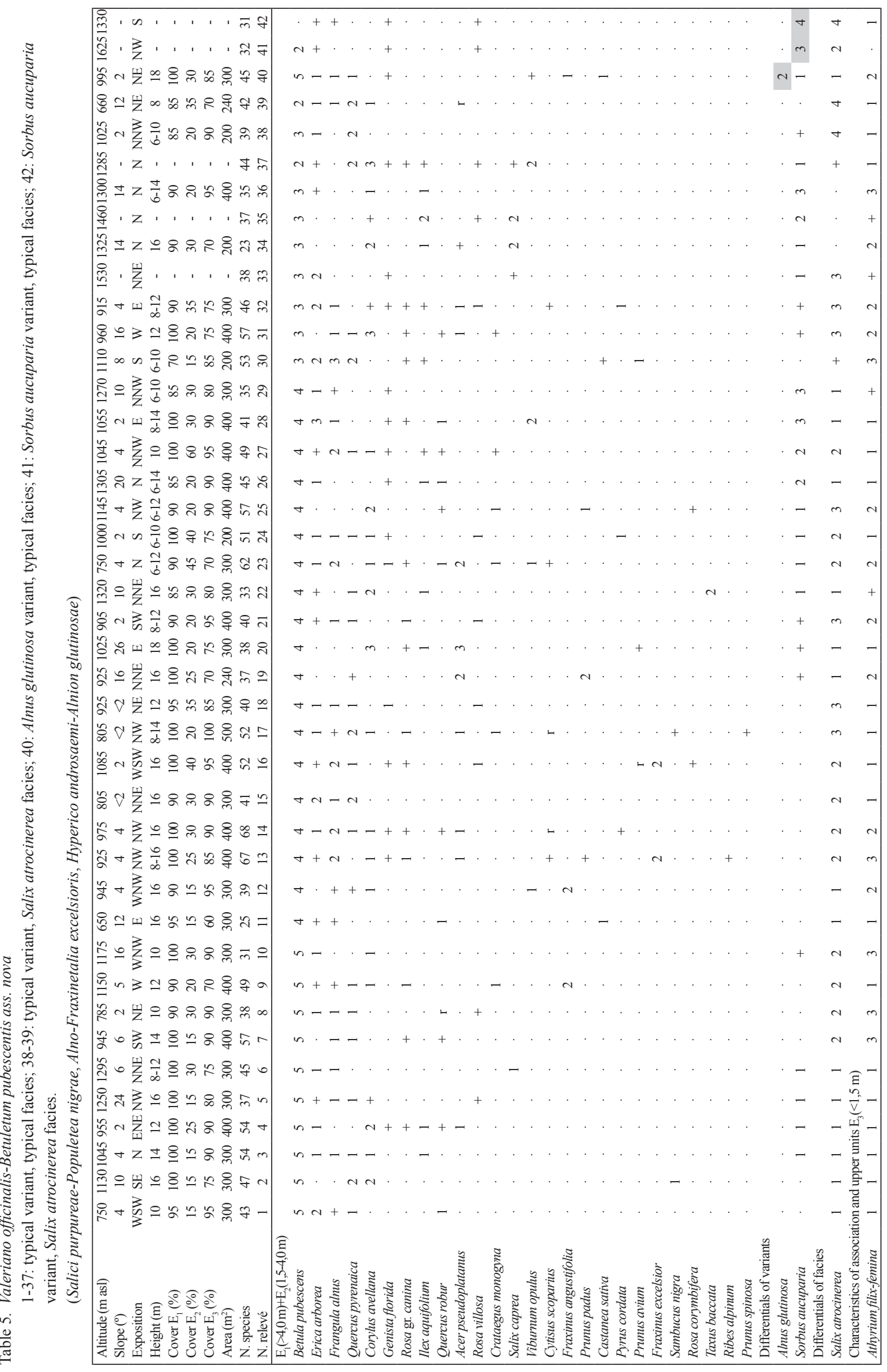




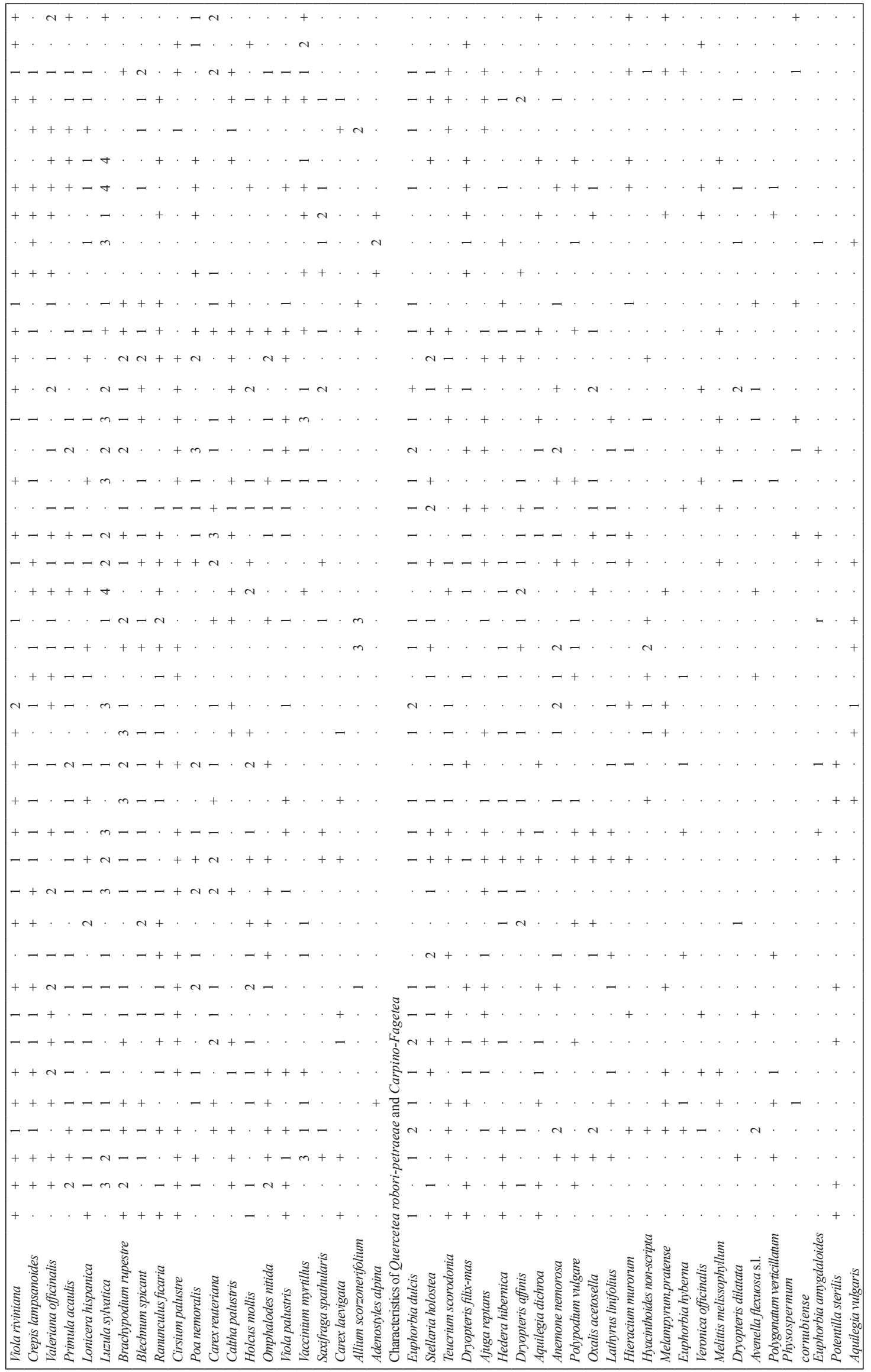




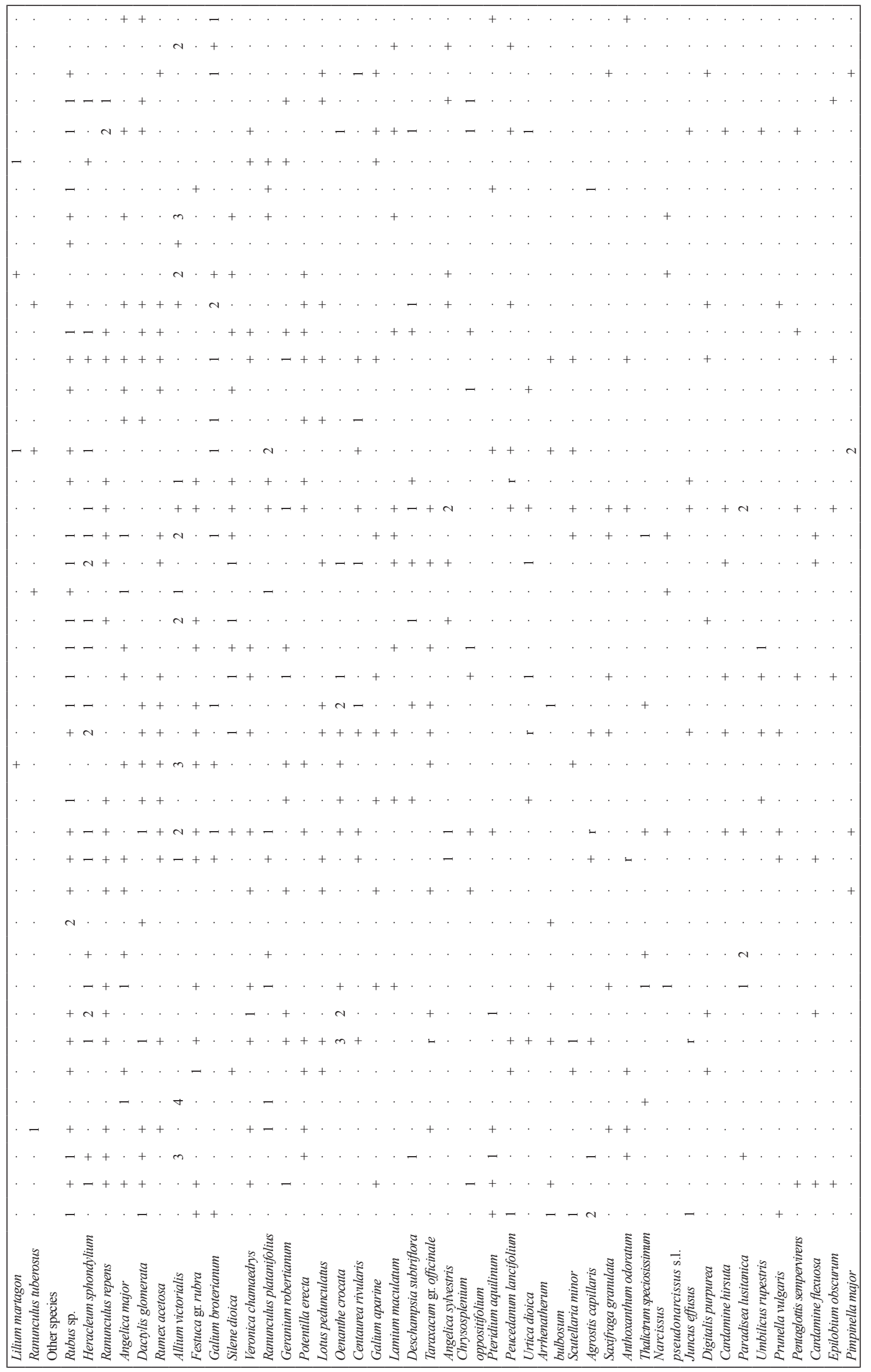




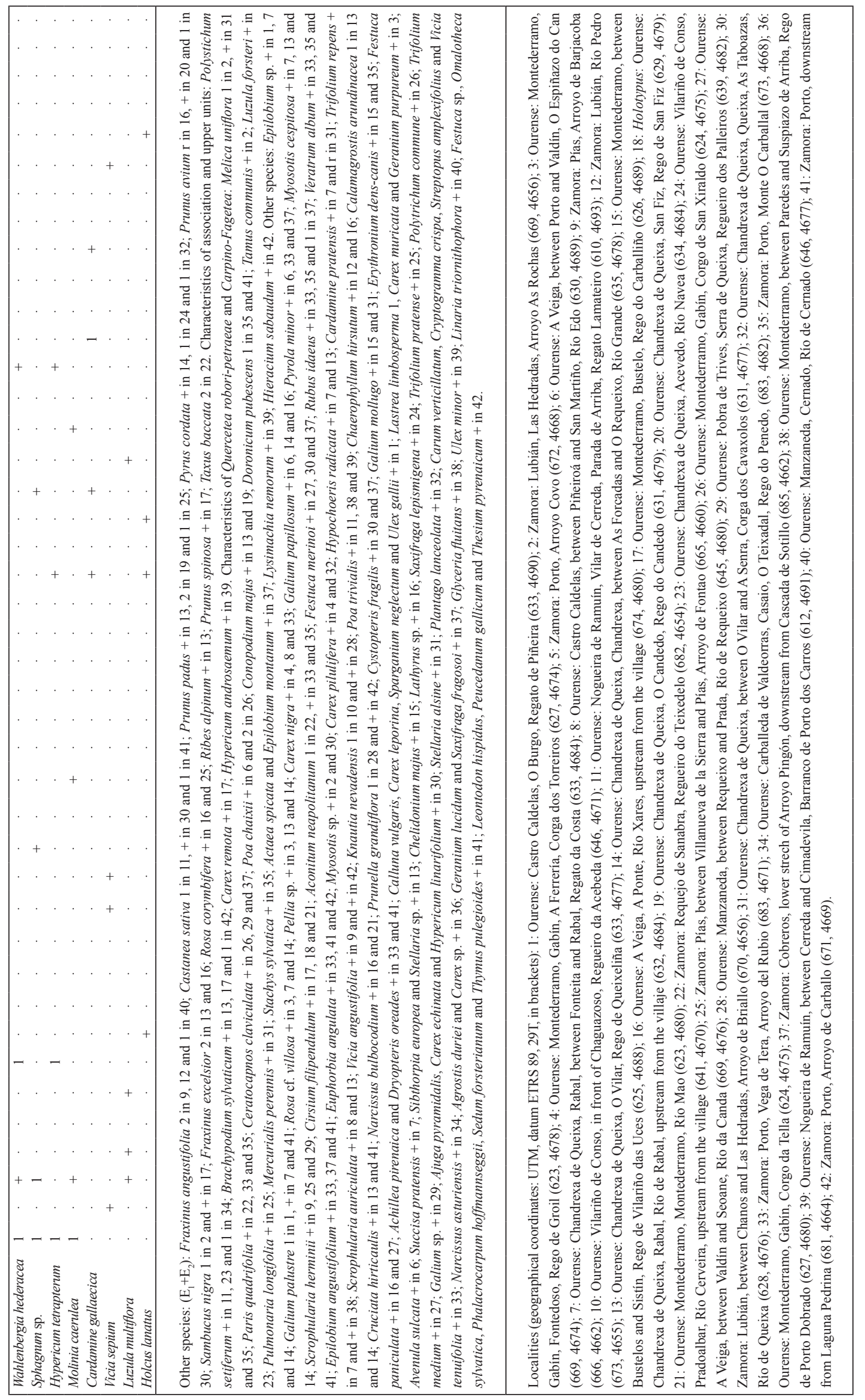


Table 6. Presence of protected taxa in the riparian birch forests of the Northwestern Iberian Peninsula. Abbreviations are: +: taxón included in the legal provision. O: taxon scarce; $\bigcirc$ : taxon frequent. HD-Annexes: annexes of the CD 92/43/EEC ('Habitats Directive'); SLTS: Spanish list of threatened species. RLTS: Regional lists of threatened species: G: Galicia region; A: Principality of Asturias; CL: Castilla \& León region. Acronyms of associations are the same of Figure 1.

\begin{tabular}{|c|c|c|c|c|c|c|c|c|c|c|}
\hline \multirow[t]{3}{*}{ Taxa } & \multicolumn{6}{|c|}{ Legal Provision } & \multicolumn{4}{|c|}{ Forest association } \\
\hline & \multicolumn{2}{|c|}{ HD-Annexes } & \multirow{2}{*}{ SLTS } & \multicolumn{3}{|c|}{ RLTS } & \multirow{2}{*}{$\mathrm{Cr}-\mathrm{Bc}$} & \multirow{2}{*}{ Vp-Bp } & \multirow{2}{*}{ Ch-Vp } & \multirow{2}{*}{ Vo-Br } \\
\hline & II & $\mathrm{V}$ & & $\mathrm{G}$ & A & $\mathrm{CL}$ & & & & \\
\hline Cardamine gallaecica & - & - & - & + & - & + & - & - & 0 & 0 \\
\hline Festuca elegans & + & - & + & - & - & - & $\mathrm{O}$ & - & $\mathrm{O}$ & $\mathrm{O}$ \\
\hline Gentiana lutea & - & + & - & - & + & + & - & - & - & $\mathrm{O}$ \\
\hline Ilex aquifolium & - & - & - & + & + & - & $\mathrm{O}$ & 0 & 0 & ○ \\
\hline Narcissus asturiensis & + & - & + & + & + & - & - & ○ & 0 & $\mathrm{O}$ \\
\hline Narcissus bulbocodium & - & + & - & - & - & + & - & $\mathrm{O}$ & - & $\mathrm{O}$ \\
\hline Narcissus nobilis & + & - & + & + & - & - & - & $\mathrm{O}$ & - & $\mathrm{O}$ \\
\hline Paradisea lusitanica & - & - & - & - & - & + & 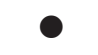 & - & - & O \\
\hline Ruscus aculeatus & - & + & - & - & - & + & O & - & - & - \\
\hline Scrophularia herminii & - & + & - & - & - & - & $\mathrm{O}$ & $\mathrm{O}$ & - & $\mathrm{O}$ \\
\hline Sphagnum spp. & - & + & - & - & - & - & 0 & - & 0 & $\mathrm{O}$ \\
\hline Taxus baccata & - & - & - & - & + & + & - & - & 0 & 0 \\
\hline
\end{tabular}

As a summary of the previous exposition, we present below the syntaxonomic scheme including the studied riparian downy birch forests:

Alno glutinosae-Populetea albae P. Fukarek et Fabijanić 1968

Alno-Fraxinetalia excelsioris Passarge 1968

Hyperico androsaemi-Alnion glutinosae (Amigo et al.1987) Biurrun et al. 2016

Saxifrago spathularis-Fraxinenion excelsioris Biurrun et al. 2016

Carici reuterianae-Betuletum celtibericae Honrado, P. Alves, Aguiar, Ortiz \& FB Caldas ex Honrado 2004

typical variant

typical facies

Salix atrocinerea facies

Alnus glutinosa variant

typical facies

Sorbus aucuparia variant

typical facies

Violo palustris-Betuletum pubescentis Rodríguez Guitián 2010

typical variant

typical facies

Salix atrocinerea facies

Alnus glutinosa variant

typical facies

Salix atrocinerea facies

Chaerophyllo hirsuti-Betuletum pubescentis ass. nova

typical variant

typical facies

Alnus glutinosa variant

typical facies

Sorbus aucuparia variant

typical facies 


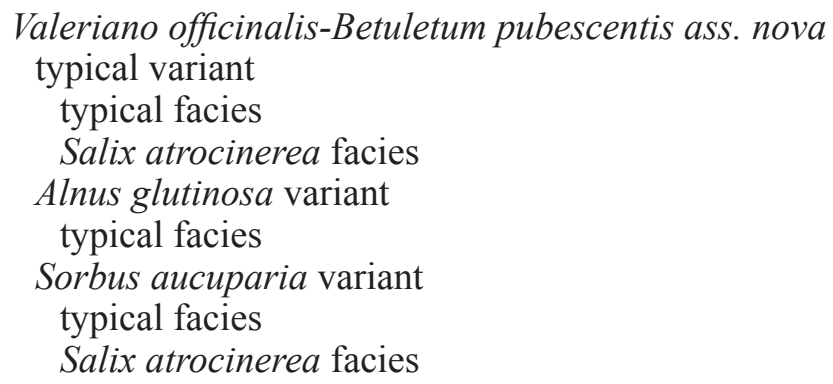

\section{Importance for conservation policies}

The studied forests are widespread in a great part of the extreme NW Iberia mountains (Figure 1) where they, as well as other riparian forest types, play important roles related to water regulation, erosion prevention and maintenance of the physicochemical characteristics of these waters (Klimo \& Hager, 2001; Douda et al., 2015; Biurrun et al., 2016).

Moreover, many of the studied forests grow inside areas belonging to the Natura 2000 Network (Figure 1). Following the ecological and floristic descriptions of the Interpretation Manual of European Union Habitats-EUR 27" (EC 2007) the riparian birch forests of the association Violo-Betuletum pubescentis have been interpreted as belonging to the habitat type 91E0* del Annex I of the CD 92/43/ECC (Habitat Directive; Rodríguez Guitián, 2010), designed as "prioritary for conservation". Considering that the last version of the Manual (EUR 28, EC 2013) did not modified the interpretation of this habitat type and that the other three associations discussed in this paper match the abiotic and biotic characteristics of this habitat, they must also be included in the 91E0* habitat type.

On the other hand, these four associations of birch forests include vascular and non-vascular plants listed in the Annex II or IV of the Habitat Directive (i.e. Festuca elegans, Gentiana lutea,
Narcissus asturiensis, N. bulbocodium, N. nobilis, Ruscus aculeatus, Scrophularia herminii or Sphagnum spp.) or in national or regional lists of protected species (Cardamine gallaecica, Ilex aquifolium, Paradisea lusitanica or Taxus baccata, Table 6). As a consequence, these forests must be considered as habitats of high conservation interest and their distribution must be taken into account when selecting sites to be part of the Natura 2000 Network or for the elaboration of their management plans.

\section{Acknowledgements}

The authors wish to thank Hugo López-Castro, Javier Pereira-Espinel and Pablo Ramil-Rego for their fieldwork help. Patricio Bariego selflessly helped us with taxonomy and to locate riparian birch forests in Sanabria (Galician-Duriensian mountains), and facilitated the access to the information collected about these forests during the implementation of the project "Cartografia Detallada de Habitats del Anexo I de la Directiva 92/43/CEE a Escala 1:10.000 en diversos Espacios incluidos en la Red Natura 2000" done by the University of Salamanca and sponsored by the Consejería de Medio Ambiente de la Junta de Castilla y León. The authors are also grateful for the the suggestions and critical review made by the referees, which helped to improve the manuscript.

\section{References}

Amigo, J. \& Romero, M.I. 1998. Abedulares de origen antrópico en Galicia: caracterización fitosociológica. Studia Bot. 17: 37-51.

Ashburner, K. \& McAllister, A. 2013. The genus Betula. A taxonomic revision of birches. A Botanical Magazine Monograph. Royal Botanic Gardens, Kew. 431 p.

Biurrun, I, Campos, J.A. García-Mijangos, I., Herrera M. \& Loidi, J. 2016. Floodplain forests of the Iberian Peninsula: Vegetation classification and climatic features. App. Veg. Sci. 19: 336-354.

Braun-Blanquet, J. 1979. Fitosociología. Bases para el estudio de las comunidades vegetales. Ed. Blume, Barcelona. 820 p. 
Castroviejo, S. 1977. Estudio sobre la vegetación de la Sierra del Invernadero (Orense). Inst. Nac. Conserv. Nat. Min. Agric., Madrid. 102 p.

Castroviejo, S. (Coord.) 1986-2013. Flora Iberica. R. Jard. Bot. CSIC, Madrid.

Costa, M., Higueras, J. \& Morla, C. 1990. Abedulares de la Sierra de San Mamede (Orense, España). Acta Bot. Malacitana 15: 253-265.

Costa, J.C., Neto, C., Aguiar, C., Capelo, J., Espírito Santo, M.D., Honrado, J., Pinto-Gomes, C., MonteiroHenriques, T., Sequeira, M. \& Lousa, M. 2012. Vascular plant communities in Portugal (Continental, the Azores and Madeira). Global Geobot. 2(1): 1-180.

Devesa, J.A., Catalán, P., Müller, J., Cebolla, C. \& Ortúñez, E. 2013. Checklist de Festuca L. (Poaceae) en la Península Ibérica. Lagascalia 33: 183-274.

Dillenberger M.S. \& Kadereit, J.W. 2012. Two new combinations in Adenostyles (Asteraceae, Senecioneae), a conspectus of the genus and key to its species and subspecies. Willdenowia 42(1): 57-61.

Douda, J., Boublik, K., Slezak, M., Biurrun, I., Nociar, J., Havrdova, A., Doudova, J., Acic, S., Brisse, H., Brunet, J., Chytry, M., Claessens, H., Csiky, J., Didukh, Y., Dimopoulos, P., Dullinger, S., FitzPatrick, U., Guisan, A., Horchler, P.J., Hrivnak, R., Jandt, U., Kazcki, Z., Kevey, B., Landucci, F., Lecomte, H., Lenoir, J., Paal, J., Paternoster, D., Pauli, H., Pielech, R., Rodwell, J.S., Roelandt, B., Svenning, J.-Ch., Sibık, J., Silc, U., Skvorc, Z., Tsiripidis, I., Tzonev, R.T., Wohlgemuth, Th. \& Zimmermann, N.E. 2015. Vegetation classification and biogeography of European floodplain forests and alder cars. App. Veg. Sci. 19: $147-163$.

EC (European Commision). 2007. Interpretation Manual of European Union Habitats-EUR 27. DG Environment. Nature and biodiversity. Brussels. $142 \mathrm{p}$.

EC (European Commision). 2013. Interpretation Manual of European Union Habitats-EUR 28. DG Environment. Nature and biodiversity. Brussels. 144 p.

Fernández Casado, M.A. \& Nava Fernández, H.S. 2015. Variabilidad morfológica de Viola palustris L. s.1., en Asturias y territorios limítrofes (Norte de España). Bol. Cien. Nat. R.I.D.E.A. 53: 189-198.

Fernández Prieto, J.A. \& Vázquez, V.M. (1987): Datos sobre los bosques asturianos orocantábricos occidentales. Lazaroa 7: 363-382.

García López, P., Lauzurica, P., Tey Quiroga, P. \& Roa Medina, A. 1992. Informe botánico del Parque Natural del Lago de Sanabria y sus alrededores. Monografía de la Red de Espacios Naturales de Castilla y León, $\mathrm{n}^{\circ}$ 4. Dir. Gral. Med. Nat. Cons. Med. Amb. Ord. Terr. Junta de Castilla y León, Valladolid. 143 p.

Gómez Manzaneque, F. (Coord.). 1997. Los bosques ibéricos. Una interpretación Geobotánica. Ed. Planeta, Barcelona. 572 p.

Gonçalves Aguiar. C.F. 2000. Flora e vegetação da Serra de Nogueira e do Parque Natural de Montesinho. Mem. Doc. (ined.). Inst. Sup. Agron. Univ. Téc. Lisboa. 659 p.

González de Paz, L. 2012. Flora y vegetación de La Cabrera Baja (León): valoración del estado de conservación. Mem. Doc. (ined.). Fac. Ci. Biol. y Amb. Univ. León. 556 p.

Guerra, J., \& Cros, R.M. (Coor.). 2006-2015. Flora Briofítica Ibérica, vols. I-V. Univ. Murcia. Soc. Esp. Briología, Murcia.

Honrado, J. 2004. De Vegetatio Lusitana Notae III. 7. Syntaxonomy of riparian woodlands in north-western Portugal (Galician-Portuguese and Juresian Sectors). Silva Lusit. 12(2): 255-256.

Honrado, J., Alves, P., Aguiar, C., Ortiz, S. \& Barreto Caldas, F. 2003. Juresian riparian birch woodlands: Carici reuterianae-Betuletum celtibericae as. nova. Silva Lusit. 11(2): 237-241.

IGME. 2017. On line Geological Map of Spain. (http://info.igme.es/visor/, last access 22 August 2016).

Izco J, Amigo J \& Guitián J. 1986. Identificación y descripción de los bosques montanos del extremo occidental de la Cordillera Cantábrica. Trab. Comp. Biol. 13: 185-203.

Klimo, E. \& Hager, H. (Eds.). 2001. The floodplain Forests in Europe. European Forest Institute Research Report 10, Leiden. The Netherlands. 267 p.

Lara, F., Garilleti, R. \& Calleja, J.A. 2004. La vegetación de ribera de la mitad norte española. Monogr. CEDEX M-81. Min. Fomento-Min. Med. Amb., Madrid. 536 p.

Morla Juaristi, C. 1985. Consideraciones acerca del paisaje vegetal y su evolución en la comarca de Trives (Orense, España). An. Geogr. 5: 189-212.

López González, G. 2001. Los árboles y arbustos de la Península Ibérica e Islas Baleares. 2 vols. Ed. Mundi-Prensa, Madrid.

Moreno, G. \& Peinado, M. 1990. Betula L. In: Castroviejo, S. (Ed.). Flora Iberica. Vol. 2. Pp. 38-43. CSIC, Madrid. 
Mucina, L., Bültmann, H., Dierßen, K., Theurillat, J.-P., Raus, Th., Čarni, A., Šumberová, K., Willner, W., Dengler, J., Gavilán García, R., Chytrý, M., Hajek, M., Di Pietro, R., Iakushenko, D., Pallas, J., Daniëls, F.J.A., Bergmeier, E., Santos Guerra, A., Ermakov, N., Valachovič, M., Schaminée, J.H.J., Lysenko, T., Didukh, Y.P., Pignatti, S., Rodwell, J.S., Capelo, J., Weber, H.E., Solomeshch, A., Dimopoulos, P., Aguiar, C., Hennekens, S.M., \& Tichý, L. 2016. Vegetation of Europe: hierarchical floristic classification system of vascular plant, bryophyte, lichen, and algal communities. App. Veg. Sci. 19 (Suppl. 1): 3-264.

Navarro Andrés, F., González Zapatero, M.A., Gallego Martín, F., Elena Roselló, J.A. Sánchez Anta, M.A. \& López Blanco, L. 1986. Alisedas salmantinas y zamoranas. Studia Bot. 5: 39-52.

Navarro, G. 1989. Contribución al conocimiento de la vegetación del Moncayo. Opusc. Bot. Pharm. Compl. 5: 5-64.

Oksanen, A., Blanchet, F.G., Kindt, R., Legendre, P., Minchin, P.R., O’Hara, B., Simpson, G.L., Solymos, P., Henry, M., Stevens, H. \& Wagner, H. 2015. Vegan: Community Ecology Package. R package version 2.3-1. https://CRAN.R-project.org/package=vegan

Ortiz, S. 1986. Series de vegetación y su zonación altitudinal en al Macizo de Pena Trevinca y Serra do Eixo. Mem. Doc. (ined.). Univ. Santiago de Compostela. 509 p.

Peinado, M. \& Moreno, G. 1989. The genus Betula (Betulaceae) in the Sistema Central (Spain). Willdenowia 18: $343-359$

Puente, E. 1985. Flora y Vegetación de la cuenca alta del Río Sil (León). Mem. Doc. (ined.). Dep. Bot. Fac. Biol. Univ. León. 536 p.

Pulgar, Í. 1999. La vegetación de la Baixa Limia y sierras del entorno. Mem. Doc. (ined.). Univ. Santiago de Compostela. 275 p.

Pulgar, Í. 2005. Guía da flora do Parque Natural Baixas Limia-Serra do Xurés. Cons. Med. Amb. Xunta de Galicia, Santiago de Compostela. 92 p.

Pulgar, Í. \& Manso, D. 2010. Datos sobre Prunus lusitanica L. (Rosaceae) en Galicia. Nova Acta Cient. Comp. (Biol.) 19: 33-47.

R Core Team. 2015. R: A language and environment for statistical computing. R Foundation for Statistical Computing, Vienna. URL http://www.R-project.org.

Ramil Rego, P., Gómez-Orellana, L., Muñoz Sobrino, C., Tereso, J.P. 2011. Dinâmica natural e transformação antrópica das floestas do noroeste ibérico. In: Tereso, J.P., Honrado, J.P., Pinto, A.T. \& Rego. F.C. (Eds.). Florestas do Norte de Portugal: História, Ecologia e Desafiosde Gestão. Pp. 14-54. InBio. Red. Invest. Biodiv. Biol. Evol. Porto.

Rivas-Martínez, S. 2007. Mapa de series, geoseries y geopermaseries de vegetación de España. Parte I. Itinera Geobot. 17: 1-436.

Rivas-Martínez, S. 2011. Mapa de series, geoseries y geopermaseries de vegetación de España. Parte II. Itinera Geobot. 18.

Rivas-Martínez, S., Díaz, T.E., Fernández-González, F., Izco, J., Loidi, J., Lousã, M. \& Penas, A. 2002. Addenda to the syntaxonomical checklist of 2001. Itinera Geobot. 15.

Rivas Martínez, S., Penas Merino, A., Díaz González, T.E., del Río González, S., Cantó Ramos, P., Herrero Cembranos, L., Pinto-Gomes, C. \& Costa, J.C. 2014. Biogeography of Spain and Portugal. Typological synopsis. Int. J. Geobot. Res. 4: 1-64.

Rivas-Martínez, S., Díaz, T.E., Fernández Prieto, J.A., Loidi, J. \& Penas, A. 1984. La vegetación de la alta montaña cantábrica. Los Picos de Europa. Ed. Leonesas, León. 300 p.

Rodríguez Guitián, M.A. 2010. Temperate riverside forests without alder trees at the NW of the Iberian Peninsula: ecology, phytosociological profile and interest for preservation policies. Lazaroa 31: 9-37.

Rodríguez Guitián, M.A. \& Bariego Hernández, P. 2009. Afinidades florísticas de los abedulares de las Sierras Segundera y Cabrera (Sanabria, NW Ibérico). In: Llamas, F. \& Acedo, C. (Coords.). Botánica Pirenaico-Cantábrica en el siglo XXI. Pp. 561-573. Publ. Universidad León, León.

Rodríguez Guitián, M.A. \& Ramil-Rego. P. 2007. Revisión de las clasificaciones climáticas aplicadas al territorio gallego desde una perspectiva biogeográfica. Rec. Rur. 1(3): 31-53.

Rodríguez Guitián, M.A. \& Ramil-Rego, P. 2008. Fitogeografía de Galicia (NW Ibérico): análisis histórico y nueva propuesta corológica. Rec. Rur. 1(4): 19-50.

Rodríguez Guitián, M.A., Amigo Vázquez, J. \& Romero Franco, R. 2000. Aportaciones sobre la interpretación, ecología y distribución de los bosques supratemplados naviano-ancarenses. Lazaroa 21: 51-71.

Rodríguez Guitián, M.A., Romero Franco, R., Real, C. \& Ferreiro da Costa, J. 2013. Descrición, cartografía e valor de conservación dos bosques da Devesa da Rogueira (Serra do Courel, NW Península Ibérica). Rec. Rur. 9: 5-34. 
Rodríguez Guitián, M.A., Romero Franco, R., Ferreiro da Costa, J. \& Díaz Varela, R.A. 2014. Tipoloxía e valor de conservación dos bosques da Reserva de Biosfera "Os Ancares Lucenses e Montes de Cervantes, Navia e Becerreá" (Lugo, Galicia, España). In: Rodríguez-Guitián, M.A. \& Rigueiro Rodríguez, A. (Coor.). A Reserva de Biosfera dos Ancares Lucenses e Montes de Cervantes, Navia e Becerreá. Rec. Rur. Ser. Cursos 7: 53-111.

Romero Rodríguez, C.M. \& Romero Cuenca, G.M. 1996. Vegetación. In: Ruíz de la Torre, J. (Dir.). Mapa Forestal de España. E 1:200.000. Hoja 3-3 Ponferrada. Pp. 79-158. Min. Med. Amb. Madrid.

Romero Rodríguez, C. \& Romero Cuenca, G.M. 2004. El paisaje forestal en los valles de Ancares y Fornela. Inst. Est. Bercianos, Ponferrada.

Ruíz de la Torre, J. 2006. Flora mayor. Organismo Autónomo Parques Nacionales. Dir. Gral. Biodiversidad. Min. Med. Amb. Madrid. 1756 p.

Silva-Pando, F.J. 2009. Los abedulares y acebedas de la Sierra de Ancares (Lugo-León, España). Actas V Congr. For. Esp. SECF-Junta Castilla y León, Ávila. http://www.secforestales.org/publicaciones/index. php/congresos/article/view File/ 7642/7565.

Tsuda, Y., Semerikov, V.L., Sebastiani, F., Vendramin, G.G. \& Lascoux. M. 2017. Multispecies genetic structure and hybridization in the Betula genus across Eurasia. Mol. Ecol. 26(2): 589-605.

Tutin, T.G. et al. (Eds.) 1964-1980. Flora Europaea. Cambrigde Univ. Press, Cambridge.

Valentine, H., Merxmüller, H. \& Schmidt, A. 1968. Viola L. In: Tutin, T.G., Heywood,V.H., Burges, N.A., Moore, D.M., Valentine, D.H., Walters, S.M. \& Webb, D.A. (Eds.): Flora Europaea, vol. 2: 270-282. Cambridge University Press. Cambridge.

van der Maarel, E.L. 1979. Transformation of cover-abundance values in phytosociology and its effects on community similarity. Vegetatio 39: 97-114.

Willis, T.J. \& Anderson, M.J. 2003. Structure of cryptic reef fish assemblages: relationships with habitat characteristics and predator density. Mar. Ecol. Progr. Ser. 257: 209-221.

Appendix 1. List of taxa present in more than four relevés with indication of their total absolute frequencies (TF) and their absolute frequencies (n) and relative frequencies (\%) in the studied riparian (R) and non-riparian (NR) birch forests. Numbers highlighted in grey correspond with exclusive taxa of one of the two groups; numbers in bold are taxa with preference for one of the groups (relative frequency $>90 \%$ )

\begin{tabular}{|l|c|c|c|c|c|}
\hline \multirow{2}{*}{ Taxon } & & \multicolumn{3}{c|}{$\mathrm{R}$} & \multicolumn{2}{c|}{$\mathrm{NR}$} \\
\cline { 3 - 7 } & & $\mathrm{TF}$ & $\%$ & $\mathrm{n}$ & $\%$ \\
\hline Acer pseudoplatanus & 56 & 39 & 69.6 & 17 & 30.4 \\
Aconitum neapolitanum & 20 & 19 & $\mathbf{9 5 . 0}$ & 1 & 5.0 \\
Adenostyles pyrenaica & 56 & 52 & $\mathbf{9 2 . 9}$ & 4 & 7.1 \\
Agrostis capillaris & 106 & 79 & 74.5 & 27 & 25.5 \\
Agrostis castellana & 7 & 0 & 0.0 & 7 & $\mathbf{1 0 0}$ \\
Ajuga pyramidalis & 5 & 5 & $\mathbf{1 0 0}$ & 0 & 0.0 \\
Ajuga reptans & 69 & 67 & $\mathbf{9 7 . 1}$ & 2 & 2.9 \\
Allium scorzonerifolium & 11 & 11 & $\mathbf{1 0 0}$ & 0 & 0.0 \\
Allium victorialis & 67 & 57 & 85.1 & 10 & 14.9 \\
Alnus glutinosa & 24 & 18 & 75.0 & 6 & 25.0 \\
Anemone albida & 21 & 17 & 81.0 & 4 & 19.0 \\
Anemone nemorosa & 100 & 65 & 65.0 & 35 & 35.0 \\
Angelica major & 95 & 91 & $\mathbf{9 5 . 8}$ & 4 & 4.2 \\
Angelica sylvestris & 13 & 12 & $\mathbf{9 2 . 3}$ & 1 & 7.7 \\
Anthoxanthum amarum & 19 & 12 & 63.2 & 7 & 36.8 \\
Anthoxanthum odoratum & 54 & 39 & 72.2 & 15 & 27.8 \\
Aquilegia dichroa & 52 & 51 & $\mathbf{9 8 . 1}$ & 1 & 1.9 \\
Aquilegia vulgaris & 29 & 24 & 82.8 & 5 & 17.2 \\
Arenaria montana & 5 & 1 & 20.0 & 4 & 80.0 \\
Arrhenatherum bulbosum & 63 & 61 & $\mathbf{9 6 . 8}$ & 2 & 3.2 \\
Astrantia major & 11 & 9 & 81.8 & 2 & 18.2 \\
Athyrium filix-femina & 225 & 215 & $\mathbf{9 5 . 6}$ & 10 & 4.4 \\
Avenula sulcata & 11 & 9 & 81.8 & 2 & 18.2 \\
Betula pubescens & & & & &
\end{tabular}

\begin{tabular}{|l|c|c|c|c|c|}
\hline \multirow{2}{*}{\multicolumn{1}{|c|}{ Taxon }} & \multirow{2}{*}{$\mathrm{TF}$} & \multicolumn{3}{c|}{$\mathrm{R}$} & \multicolumn{2}{c|}{$\mathrm{NR}$} \\
\cline { 3 - 7 } & & $\mathrm{n}$ & $\%$ & $\mathrm{n}$ & $\%$ \\
\hline Lilium martagon & 31 & 17 & 54.8 & 14 & 45.2 \\
Lonicera hispanica & 150 & 150 & $\mathbf{1 0 0}$ & 0 & 0.0 \\
Lonicera periclymenum & 43 & 1 & 2.3 & 42 & $\mathbf{9 7 . 7}$ \\
Lotus pedunculatus & 40 & 39 & $\mathbf{9 7 . 5}$ & 1 & 2.5 \\
Luzula multiflora & 10 & 10 & $\mathbf{1 0 0}$ & 0 & 0.0 \\
Luzula sylvatica & 186 & 134 & 72.0 & 52 & 28.0 \\
Lysimachia nemorum & 13 & 13 & $\mathbf{1 0 0}$ & 0 & 0.0 \\
Lysimachia vulgaris & 7 & 7 & $\mathbf{1 0 0}$ & 0 & 0.0 \\
Lythrum salicaria & 7 & 7 & $\mathbf{1 0 0}$ & 0 & 0.0 \\
Melampyrum pratense & 70 & 30 & 42.9 & 40 & 57.1 \\
Melica uniflora & 15 & 11 & 73.3 & 4 & 26.7 \\
Melittis melissophyllum & 19 & 17 & 89.5 & 2 & 10.5 \\
Mercurialis perennis & 17 & 13 & 76.5 & 4 & 23.5 \\
Milium effusum & 9 & 7 & 77.8 & 2 & 22.2 \\
Molinia caerulea & 27 & 26 & $\mathbf{9 6 . 3}$ & 1 & 3.7 \\
Myosotis cespitosa & 9 & 9 & $\mathbf{1 0 0}$ & 0 & 0.0 \\
Myosotis martini & 8 & 8 & $\mathbf{1 0 0}$ & 0 & 0.0 \\
Myosotis sylvatica & 5 & 0 & 0.0 & 5 & $\mathbf{1 0 0}$ \\
Narcissus asturiensis & 27 & 27 & $\mathbf{1 0 0}$ & 0 & 0.0 \\
Narcissus bulbocodium & 5 & 5 & $\mathbf{1 0 0}$ & 0 & 0.0 \\
Narcissus pseudonarcissus s.1. & 13 & 12 & $\mathbf{9 2 . 3}$ & 1 & 7.7 \\
Narcissus triandrus & 9 & 4 & 44.4 & 5 & 55.6 \\
Oenanthe crocata & 76 & 76 & $\mathbf{1 0 0}$ & 0 & 0.0 \\
Omphalodes nitida & 121 & 86.4 & 19 & 13.6 \\
\hline
\end{tabular}




\begin{tabular}{|c|c|c|c|c|c|}
\hline \multirow{2}{*}{ Taxon } & \multirow{2}{*}{$\mathrm{TF}$} & \multicolumn{2}{|c|}{$\mathrm{R}$} & \multicolumn{2}{|c|}{ NR } \\
\hline & & $\mathrm{n}$ & $\%$ & $\mathrm{n}$ & $\%$ \\
\hline Blechnum spicant & 219 & 181 & 82.6 & 38 & 17.4 \\
\hline Brachypodium rupestre & 117 & 112 & 95.7 & 5 & 4.3 \\
\hline Brachypodium sylvaticum & 11 & 9 & 81.8 & 2 & 18.2 \\
\hline Calamagrostis arundinacea & 10 & 10 & 100 & 0 & 0.0 \\
\hline Calluna vulgaris & 10 & 5 & 50.0 & 5 & 50.0 \\
\hline Caltha palustris & 85 & 82 & 96.5 & 3 & 3.5 \\
\hline Cardamine flexuosa & 9 & 9 & 100 & 0 & 0.0 \\
\hline Cardamine gallaecica & 15 & 15 & 100 & 0 & 0.0 \\
\hline Cardamine hirsuta & 15 & 15 & 100 & 0 & 0.0 \\
\hline Cardamine pratensis & 27 & 27 & 100 & 0 & 0.0 \\
\hline Carex divulsa & 6 & 6 & 100 & 0 & 0.0 \\
\hline Carex echinata & 14 & 14 & 100 & 0 & 0.0 \\
\hline Carex laevigata & 81 & 80 & 98.8 & 1 & 1.2 \\
\hline Carex pilulifera & 20 & 13 & 65.0 & 7 & 35.0 \\
\hline Carex remota & 11 & 9 & 81.8 & 2 & 18.2 \\
\hline Carex reuteriana & 91 & 91 & 100 & 0 & 0.0 \\
\hline Castanea sativa & 41 & 22 & 53.7 & 19 & 46.3 \\
\hline Centaurea nigra & 67 & 64 & 95.5 & 3 & 4.5 \\
\hline Ceratocapnos claviculata & 18 & 12 & 66.7 & 6 & 33.3 \\
\hline Chaerophyllum hirsutum & 47 & 40 & 85.1 & 7 & 14.9 \\
\hline Chrysosplenium oppositifolium & 38 & 38 & 100 & 0 & 0.0 \\
\hline Cicerbita plumieri & 11 & 9 & 81.8 & 2 & 18.2 \\
\hline Cirsium filipendulum & 21 & 17 & 81.0 & 4 & 19.0 \\
\hline Cirsium palustre & 114 & 109 & 95.6 & 5 & 4.4 \\
\hline Conopodium majus & 11 & 6 & 54.5 & 5 & 45.5 \\
\hline Corylus avellana & 118 & 92 & 78.0 & 26 & 22.0 \\
\hline Crataegus monogyna & 31 & 24 & 77.4 & 7 & 22.6 \\
\hline Crepis lampsanoides & 164 & 129 & 78.7 & 35 & 21.3 \\
\hline Crocus serotinus & 49 & 49 & 100 & 0 & 0.0 \\
\hline Cytisus scoparius & 41 & 32 & 78.0 & 9 & 22.0 \\
\hline Cytisus striatus & 9 & 8 & 88.9 & 1 & 11.1 \\
\hline Daboecia cantabrica & 10 & 5 & 50.0 & 5 & 50.0 \\
\hline Dactylis glomerata & 101 & 94 & 93.1 & 7 & 6.9 \\
\hline Dactylorhiza maculata & 6 & 6 & 100 & 0 & 0.0 \\
\hline Danthonia decumbens & 7 & 3 & 42.9 & 4 & 57.1 \\
\hline Daphne laureola & 14 & 5 & 35.7 & 9 & 64.3 \\
\hline Deschampsia flexuosa & 97 & 62 & 63.9 & 35 & 36.1 \\
\hline Deschampsia subtriflora & 112 & 108 & 96.4 & 4 & 3.6 \\
\hline Digitalis purpurea & 98 & 74 & 75.5 & 24 & 24.5 \\
\hline Doronicum pubescens & 24 & 11 & 45.8 & 13 & 54.2 \\
\hline Dryopteris aemula & 19 & 19 & 100 & 0 & 0.0 \\
\hline Dryopteris affinis & 162 & 144 & 88.9 & 18 & 11.1 \\
\hline Dryopteris dilatata & 118 & 80 & 67.8 & 38 & 32.2 \\
\hline Dryopteris expansa & 5 & 1 & 20.0 & 4 & 80.0 \\
\hline Dryopteris filix-mas & 83 & 56 & 67.5 & 27 & 32.5 \\
\hline Epilobium montanum & 8 & 8 & 100 & 0 & 0.0 \\
\hline Epilobium obscurum & 25 & 25 & 100 & 0 & 0.0 \\
\hline Erica arborea & 264 & 190 & 72.0 & 74 & 28.0 \\
\hline Erica vagans & 7 & 4 & 57.1 & 3 & 42.9 \\
\hline Erythronium dens-canis & 9 & 6 & 66.7 & 3 & 33.3 \\
\hline Euphorbia amygdaloides & 42 & 32 & 76.2 & 10 & 23.8 \\
\hline Euphorbia angulata & 8 & 6 & 75.0 & 2 & 25.0 \\
\hline Euphorbia dulcis & 169 & 154 & 91.1 & 15 & 8.9 \\
\hline Euphorbia hyberna & 56 & 42 & 75.0 & 14 & 25.0 \\
\hline Fagus sylvatica & 20 & 11 & 55.0 & 9 & 45.0 \\
\hline
\end{tabular}

\begin{tabular}{|c|c|c|c|c|c|}
\hline \multirow{2}{*}{ Taxon } & \multirow{2}{*}{ TF } & \multicolumn{2}{|c|}{$\mathrm{R}$} & \multicolumn{2}{|c|}{ NR } \\
\hline & & $\mathrm{n}$ & $\%$ & $\mathrm{n}$ & $\%$ \\
\hline Ornithogalum pyrenaicum & 7 & 6 & 85.7 & 1 & 14.3 \\
\hline Osmunda regalis & 35 & 35 & 100 & 0 & 0.0 \\
\hline Oxalis acetosella & 116 & 85 & 73.3 & 31 & 26.7 \\
\hline Paradisea lusitanica & 19 & 19 & 100 & 0 & 0.0 \\
\hline Paris quadrifolia & 6 & 6 & 100 & 0 & 0.0 \\
\hline Pentaglottis sempervirens & 12 & 12 & 100 & 0 & 0.0 \\
\hline Peucedanum lancifolium & 34 & 34 & 100 & 0 & 0.0 \\
\hline Physospermum cornubiense & 24 & 19 & 79.2 & 5 & 20.8 \\
\hline Poa chaixii & 23 & 11 & 47.8 & 12 & 52.2 \\
\hline Poa nemoralis & 84 & 63 & 75.0 & 21 & 25.0 \\
\hline Poa trivialis & 6 & 6 & 100 & 0 & 0.0 \\
\hline Polygonatum odoratum & 6 & 4 & 66.7 & 2 & 33.3 \\
\hline Polygonatum verticillatum & 49 & 36 & 73.5 & 13 & 26.5 \\
\hline Polygonum bistorta & 5 & 5 & 100 & 0 & 0.0 \\
\hline Polypodium vulgare & 87 & 78 & 89.7 & 9 & 10.3 \\
\hline Polystichum aculeatum & 10 & 7 & 70.0 & 3 & 30.0 \\
\hline Polystichum setiferum & 15 & 13 & 86.7 & 2 & 13.3 \\
\hline Polytrichum commune & 17 & 17 & 100 & 0 & 0.0 \\
\hline Potentilla erecta & 59 & 51 & 86.4 & 8 & 13.6 \\
\hline Potentilla sterilis & 17 & 17 & 100 & 0 & 0.0 \\
\hline Primula acaulis & 83 & 78 & 94.0 & 5 & 6.0 \\
\hline Primula columnae & 5 & 4 & 80.0 & 1 & 20.0 \\
\hline Prunella grandiflora & 11 & 11 & 100 & 0 & 0.0 \\
\hline Prunella vulgaris & 40 & 40 & 100 & 0 & 0.0 \\
\hline Prunus avium & 11 & 9 & 81.8 & 2 & 18.2 \\
\hline Prunus padus & 14 & 11 & 78.6 & 3 & 21.4 \\
\hline Prunus spinosa & 6 & 1 & 16.7 & 5 & 83.3 \\
\hline Pseudarrhenatherum longifolium & 10 & 6 & 60.0 & 4 & 40.0 \\
\hline Pteridium aquilinum & 168 & 103 & 61.3 & 65 & 38.7 \\
\hline Pulmonaria longifolia & 13 & 13 & 100 & 0 & 0.0 \\
\hline Pyrola minor & 12 & 12 & 100 & 0 & 0.0 \\
\hline Pyrus cordata & 49 & 46 & 93.9 & 3 & 6.1 \\
\hline Quercus petraea & 19 & 14 & 73.7 & 5 & 26.3 \\
\hline Quercus pyrenaica & 59 & 53 & 89.8 & 6 & 10.2 \\
\hline Quercus robur & 144 & 105 & 72.9 & 39 & 27.1 \\
\hline Quercus robur $x$ Q. petraea & 10 & 2 & 20.0 & 8 & 80.0 \\
\hline Ranunculus ficaria & 56 & 56 & 100 & 0 & 0.0 \\
\hline Ranunculus platanifolius & 49 & 46 & 93.9 & 3 & 6.1 \\
\hline Ranunculus repens & 95 & 95 & 100 & 0 & 0.0 \\
\hline Ranunculus tuberosus & 28 & 20 & 71.4 & 8 & 28.6 \\
\hline Rosa corymbifera & 5 & 4 & 80.0 & 1 & 20.0 \\
\hline Rosa villosa & 18 & 18 & 100 & 0 & 0.0 \\
\hline Rubus idaeus & 13 & 12 & 92.3 & 1 & 7.7 \\
\hline Rubus plicatus & 6 & 6 & 100 & 0 & 0.0 \\
\hline Rumex obtusifolius & 6 & 6 & 100 & 0 & 0.0 \\
\hline Rumex acetosa & 65 & 59 & 90.8 & 6 & 9.2 \\
\hline Salix atrocinerea & 227 & 210 & 92.5 & 17 & 7.5 \\
\hline Salix caprea & 30 & 25 & 83.3 & 5 & 16.7 \\
\hline Sambucus nigra & 21 & 17 & 81.0 & 4 & 19.0 \\
\hline Sanicula europaea & 12 & 8 & 66.7 & 4 & 33.3 \\
\hline Saxifraga granulata & 7 & 7 & 100 & 0 & 0.0 \\
\hline Saxifraga lepismigena & 33 & 33 & 100 & 0 & 0.0 \\
\hline Saxifraga spathularis & 145 & 98 & 67.6 & 47 & 32.4 \\
\hline Scrophularia alpestris & 17 & 17 & 100 & 0 & 0.0 \\
\hline Scrophularia auriculata & 25 & 25 & 100 & 0 & 0.0 \\
\hline
\end{tabular}




\begin{tabular}{|c|c|c|c|c|c|}
\hline \multirow{2}{*}{ Taxon } & \multirow{2}{*}{$\mathrm{TF}$} & \multicolumn{2}{|c|}{$\mathrm{R}$} & \multicolumn{2}{|c|}{ NR } \\
\hline & & $\mathrm{n}$ & $\%$ & $\mathrm{n}$ & $\%$ \\
\hline Festuca merinoi & 11 & 9 & 81.8 & 2 & 18.2 \\
\hline Fragaria vesca & 14 & 11 & 78.6 & 3 & 21.4 \\
\hline Frangula alnus & 135 & 121 & 89.6 & 14 & 10.4 \\
\hline Fraxinus angustifolia & 6 & 5 & 83.3 & 1 & 16.7 \\
\hline Fraxinus excelsior & 25 & 20 & 80.0 & 5 & 20.0 \\
\hline Galium aparine & 18 & 16 & 88.9 & 2 & 11.1 \\
\hline Galium broterianum & 38 & 38 & 100 & 0 & 0.0 \\
\hline Galium mollugo & 8 & 3 & 37.5 & 5 & 62.5 \\
\hline Galium odoratum & 8 & 5 & 62.5 & 3 & 37.5 \\
\hline Galium palustre & 26 & 26 & 100 & 0 & 0.0 \\
\hline Galium papillosum & 11 & 11 & 100 & 0 & 0,0 \\
\hline Galium rotundifolium & 6 & 3 & 50,0 & 3 & 50,0 \\
\hline Galium saxatile & 23 & 6 & 26,1 & 17 & 73,9 \\
\hline Genista florida & 86 & 57 & 66,3 & 29 & 33,7 \\
\hline Gentiana aurantiaca & 7 & 1 & 14,3 & 6 & 85,7 \\
\hline Geranium robertianum & 45 & 43 & 95,6 & 2 & 4,4 \\
\hline Glandora prostrata & 5 & 4 & 80,0 & 1 & 20,0 \\
\hline Hedera hibernica & 149 & 118 & 79,2 & 31 & 20,8 \\
\hline Helleborus foetidus & 9 & 7 & 77,8 & 2 & 22,2 \\
\hline Helleborus occidentalis & 6 & 2 & 33,3 & 4 & 66,7 \\
\hline Heracleum sphondylium & 61 & 60 & $\mathbf{9 8 , 4}$ & 1 & 1,6 \\
\hline Hieracium sabaudum & 8 & 6 & 75,0 & 2 & 25,0 \\
\hline Holcus lanatus & 22 & 20 & 90,9 & 2 & 9,1 \\
\hline Holcus mollis & 205 & 157 & 76,6 & 48 & 23,4 \\
\hline Hyacinthoides non-scripta & 43 & 32 & 74,4 & 11 & 25,6 \\
\hline Hyacinthoides paivae & 13 & 12 & 92,3 & 1 & 7,7 \\
\hline Hypericum androsaemum & 10 & 10 & 100 & 0 & 0,0 \\
\hline Hypericum pulchrum & 5 & 1 & 20,0 & 4 & 80,0 \\
\hline Hypericum tetrapterum & 7 & 7 & 100 & 0 & 0,0 \\
\hline Ilex aquifolium & 127 & 82 & 64,6 & 45 & 35,4 \\
\hline Jasione montana & 7 & 2 & 28,6 & 5 & 71,4 \\
\hline Juncus effusus & 39 & 39 & 100 & 0 & 0,0 \\
\hline Lamium maculatum & 42 & 37 & 88,1 & 5 & 11,9 \\
\hline Lasepitium merinoi & 5 & 5 & 100 & 0 & 0,0 \\
\hline Laserpitium thalictrifolium & 5 & 3 & 60,0 & 2 & 40,0 \\
\hline Lastrea limbosperma & 50 & 47 & 94,0 & 3 & 6,0 \\
\hline Lathyrus linifolius & 40 & 40 & 100 & 0 & 0,0 \\
\hline
\end{tabular}

\begin{tabular}{|c|c|c|c|c|c|}
\hline \multirow{2}{*}{ Taxon } & \multirow{2}{*}{$\mathrm{TF}$} & \multicolumn{2}{|c|}{$\mathrm{R}$} & \multicolumn{2}{|c|}{ NR } \\
\hline & & $\mathrm{n}$ & $\%$ & $\mathrm{n}$ & $\%$ \\
\hline Scrophularia herminii & 11 & 11 & 100 & 0 & 0.0 \\
\hline Scutellaria minor & 26 & 24 & 92.3 & 2 & 7.7 \\
\hline Senecio aquaticus & 6 & 6 & 100 & 0 & 0.0 \\
\hline Senecio nemorensis & 52 & 45 & 86.5 & 7 & 13.5 \\
\hline Sibthorpia europaea & 8 & 8 & 100 & 0 & 0.0 \\
\hline Silene dioica & 47 & 45 & 95.7 & 2 & 4.3 \\
\hline Silene vulgaris & 5 & 4 & 80.0 & 1 & 20.0 \\
\hline Solidago virgaurea & 12 & 7 & 58.3 & 5 & 41.7 \\
\hline Sorbus aucuparia & 173 & 95 & 54.9 & 78 & 45.1 \\
\hline Stachys officinalis & 13 & 13 & 100 & 0 & 0.0 \\
\hline Stellaria alsine & 5 & 5 & 100 & 0 & 0,0 \\
\hline Stellaria holostea & 163 & 105 & 64,4 & 58 & 35,6 \\
\hline Stellaria montana & 5 & 3 & 60,0 & 2 & 40,0 \\
\hline Succisa pratensis & 12 & 12 & 100 & 0 & 0,0 \\
\hline Taxus baccata & 17 & 3 & 17,6 & 14 & 82,4 \\
\hline Teucrium scorodonia & 189 & 141 & 74,6 & 48 & 25,4 \\
\hline Thalictrum minus & 7 & 7 & 100 & 0 & 0,0 \\
\hline Thalictrum speciosissimum & 11 & 11 & 100 & 0 & 0,0 \\
\hline Ulex europaeus & 6 & 4 & 66,7 & 2 & 33,3 \\
\hline Ulex galli & 12 & 7 & 58,3 & 5 & 41,7 \\
\hline Ulex minor & 6 & 6 & 100 & 0 & 0,0 \\
\hline Umbilicus rupestris & 16 & 16 & 100 & 0 & 0,0 \\
\hline Urtica dioica & 21 & 21 & 100 & 0 & 0,0 \\
\hline Vaccinium myrtillus & 181 & 109 & 60,2 & 72 & 39,8 \\
\hline Valeriana dioica & 10 & 5 & 50,0 & 5 & 50,0 \\
\hline Valeriana montana & 41 & 24 & 58,5 & 17 & 41,5 \\
\hline Valeriana officinalis & 34 & 34 & 100 & 0 & 0,0 \\
\hline Valeriana pyrenaica & 19 & 17 & 89,5 & 2 & 10,5 \\
\hline Veratrum album & 11 & 6 & 54,5 & 5 & 45,5 \\
\hline Veronica chamaedrys & 41 & 36 & 87,8 & 5 & 12,2 \\
\hline Veronica montana & 6 & 3 & 50,0 & 3 & 50,0 \\
\hline Veronica officinalis & 37 & 31 & 83,8 & 6 & 16,2 \\
\hline Viburnum opulus & 7 & 6 & 85,7 & 1 & 14,3 \\
\hline Vicia sepium & 21 & 21 & 100 & 0 & 0,0 \\
\hline Viola palustris & 139 & 134 & 96,4 & 5 & 3,6 \\
\hline Viola riviniana & 143 & 115 & 80,4 & 28 & 19,6 \\
\hline Wahlenbergia hederacea & 25 & 25 & 100 & 0 & 0,0 \\
\hline
\end{tabular}

